\title{
$R$-matrices in rime
}

\author{
Oleg Ogievetsky ${ }^{1, *, \dagger}$ and Todor Popov ${ }^{2}$
}

\author{
${ }^{1}$ Centre de Physique Théorique, Luminy, 13288 Marseille, France \\ ${ }^{2}$ Institute for Nuclear Research and Nuclear Energy, \\ Bulgarian Academy of Sciences, Sofia, BG-1784, Bulgaria
}

\begin{abstract}
We replace the ice Ansatz on matrix solutions of the Yang-Baxter equation by a weaker condition which we call rime. Rime solutions include the standard Drinfeld-Jimbo $R$-matrix. Solutions of the YangBaxter equation within the rime Ansatz which are maximally different from the standard one we call strict rime. A strict rime non-unitary solution is parameterized by a projective vector $\vec{\phi}$. We show that in the finite dimension this solution transforms to the Cremmer-Gervais $R$-matrix by a change of basis with a matrix containing symmetric functions in the components of $\vec{\phi}$. A strict unitary solution (the rime Ansatz is well adapted for taking a unitary limit) in the finite dimension is shown to be equivalent to a quantization of a classical "boundary" $r$-matrix of Gerstenhaber and Giaquinto. We analyze the structure of the elementary rime blocks and find, as a by-product, that all non-standard $R$-matrices of $G L(1 \mid 1)$-type can be uniformly described in a rime form. We discuss then connections of the classical rime solutions with the Bézout operators. The Bézout operators satisfy the (non-)homogeneous associative classical Yang-Baxter equation which is related to the Rota-Baxter operators. We calculate the Rota-Baxter operators corresponding to the Bézout operators. We classify the rime Poisson brackets: they form a three-dimensional pencil. A normal form of each individual member of
\end{abstract}

e-print archive: http://lanl.arXiv.org/abs/0704.1947

* On leave of absence from P.N. Lebedev Physical Institute, Theoretical Department, Leninsky prospekt 53, 119991 Moscow, Russia.

${ }^{\dagger}$ Unité Mixte de Recherche (UMR 6207) du CNRS et des Universités Aix-Marseille I, Aix-Marseille II et du Sud Toulon - Var; laboratoire affilié à la FRUMAM (FR 2291). 
the pencil depends on the discriminant of a certain quadratic polynomial. We also classify orderable quadratic rime associative algebras. For the standard Drinfeld--Jimbo solution, there is a choice of the multiparameters, for which it can be non-trivially rimed. However, not every Belavin-Drinfeld triple admits a choice of the multiparameters for which it can be rimed. We give a minimal example.

\section{Contents}

1 From ice to rime 441

2 Rime Yang-Baxter solutions 446

$2.1 \quad$ Non-unitary rime $R$-matrices $\quad 446$

$2.2 \quad$ Unitary rime $R$-matrices $\quad 447$

$\begin{array}{lll}2.3 & \text { Properties } & 448\end{array}$

$3 \quad$ Rime and Cremmer-Gervais $R$-matrices 451

4 Classical rime $r$-matrices $\quad 455$

4.1 Non-skew-symmetric case 455

$\begin{array}{lll}4.2 & \text { BD triples } & 457\end{array}$

$\begin{array}{lll}4.3 & \text { Skew-symmetric case } & 460\end{array}$

5 Bézout operators 465

5.1 Non-homogeneous associative classical Yang-Baxter equation $\quad 467$

$\begin{array}{lll}5.2 & \text { Linear quantization } & 470\end{array}$

$\begin{array}{lll}5.3 & \text { Algebraic meaning } & 471\end{array}$

5.4 Rota-Baxter operators $\quad 472$

$5.5 *$-multiplication $\quad 475$ 
6 Rime Poisson brackets 478

$\begin{array}{lll}6.1 & \text { Rime pencil } & 478\end{array}$

$\begin{array}{lll}6.2 & \text { Invariance } & 482\end{array}$

$\begin{array}{lll}\text { 6.3 Normal form } & 487\end{array}$

$7 \quad$ Orderable quadratic rime associative algebras 489

$\begin{array}{ll}\text { Acknowledgments } & 491\end{array}$

Appendix A. Equations 492

Appendix B. Blocks 493

$\begin{array}{lll}\text { B.1 Solutions } & 493\end{array}$

B.2 $\mathrm{GL}(2)$ and $\mathrm{GL}(1 \mid 1) R$-matrices 496

B.3 Riming 499

Appendix C. Rimeless triple 501

$\begin{array}{ll}\text { References } & 503\end{array}$

\section{From ice to rime}

A well-known class of solutions $\hat{R} \in \operatorname{End}(V \otimes V), V$ is a vector space, of the Yang-Baxter equation $Y B(\hat{R})=0$, where

$$
Y B(\hat{R}):=(\hat{R} \otimes \mathbb{1})(\mathbb{1} \otimes \hat{R})(\hat{R} \otimes \mathbb{1})-(\mathbb{1} \otimes \hat{R})(\hat{R} \otimes \mathbb{1})(\mathbb{1} \otimes \hat{R}),
$$

is characterized by the so-called ice condition (see lectures [21] for details), which says that $\hat{R}_{k l}^{i j}$ can be different from zero only if the set of the upper and the set of the lower indices coincide,

$$
\hat{R}_{k l}^{i j} \neq 0 \quad \Rightarrow \quad\{i, j\} \equiv\{k, l\} .
$$

We introduce the "rime" Ansatz, relaxing the ice condition: the entry $\hat{R}_{k l}^{i j}$ can be different from zero if the set of the lower indices is a subset of the 
set of the upper indices,

$$
\hat{R}_{k l}^{i j} \neq 0 \quad \Rightarrow \quad\{k, l\} \subset\{i, j\}
$$

Matrices for which it holds will be referred to as "rime" matrices. Figuratively, in the rime, in contrast to the ice, situation, putting an apple and a banana in a fridge, there is a non-zero amplitude to find next morning two apples instead (but never an apple and an orange).

The Yang-Baxter equation for a matrix $\hat{R}$ is equivalent to the equality of two different reorderings of $x^{i} y^{j} z^{k}$, using $x^{i} y^{j}=\hat{R}_{k l}^{i j} y^{k} x^{l}, x^{i} z^{j}=\hat{R}_{k l}^{i j} z^{k} x^{l}$ and $y^{i} z^{j}=\hat{R}_{k l}^{i j} z^{k} y^{l}$, to the form $z^{\bullet} y^{\bullet} x^{\bullet}$. One of advantages of the rime Ansatz is that only indices $i, j$ and $k$ appear in the latter expression. Another advantage is that for fixed values of $i$ and $j$, the elements $x^{\bullet}$ and $y^{\bullet}$ with these values of indices form a subsystem.

A rime $R$-matrix has the following structure:

$$
\hat{R}_{k l}^{i j}=\alpha_{i j} \delta_{l}^{i} \delta_{k}^{j}+\beta_{i j} \delta_{k}^{i} \delta_{l}^{j}+\gamma_{i j} \delta_{k}^{i} \delta_{l}^{i}+\gamma_{i j}^{\prime} \delta_{k}^{j} \delta_{l}^{j} \quad \text { (no summation) }
$$

To avoid redundancy, fix $\beta_{i i}=0, \gamma_{i i}=0=\gamma_{i i}^{\prime}$. We denote by $\alpha_{i}$ the diagonal elements $\hat{R}_{i i}^{i i}, \alpha_{i}=\alpha_{i i}$. Throughout the text we shall assume that the matrix $\hat{R}$ is invertible which, in particular, implies that $\alpha_{i} \neq 0$ for all $i$.

The order of growth of the number of unknowns in the Yang-Baxter system for a rime matrix is $n^{2}$, where $n=\operatorname{dim} V$.

Arbitrary permutations and rescalings of coordinates preserve the rime condition.

The ice and rime matrices are made of $4 \times 4$ elementary building blocks, respectively,

$$
\hat{R}^{\text {ice }}=\left(\begin{array}{cccc}
\alpha_{1} & 0 & 0 & 0 \\
0 & \beta_{12} & \alpha_{12} & 0 \\
0 & \alpha_{21} & \beta_{21} & 0 \\
0 & 0 & 0 & \alpha_{2}
\end{array}\right) \quad \text { and } \quad \hat{R}^{\text {rime }}=\left(\begin{array}{cccc}
\alpha_{1} & 0 & 0 & 0 \\
\gamma_{12} & \beta_{12} & \alpha_{12} & \gamma_{12}^{\prime} \\
\gamma_{21}^{\prime} & \alpha_{21} & \beta_{21} & \gamma_{21} \\
0 & 0 & 0 & \alpha_{2}
\end{array}\right)
$$

In Appendix B we analyze the structure of the $4 \times 4$ rime blocks.

We call a rime matrix strict if $\alpha_{i j} \gamma_{i j} \neq 0 \forall i$ and $j, i \neq j$. Note that strict rime matrices are necessarily not ice. 
Proposition 1.1. Let $\hat{R}$ be a rime matrix (1.4). Then $\hat{R}$ is a solution of the Yang-Baxter equation if it is of the form

$$
\hat{R}_{k l}^{i j}=\left(1-\beta_{j i}\right) \delta_{l}^{i} \delta_{k}^{j}+\beta_{i j} \delta_{k}^{i} \delta_{l}^{j}+\gamma_{i j} \delta_{k}^{i} \delta_{l}^{i}-\gamma_{j i} \delta_{k}^{j} \delta_{l}^{j}
$$

where $\beta_{i j}$ and $\gamma_{i j}$ satisfy the system

$$
\begin{gathered}
\beta_{i j} \beta_{j i}=\gamma_{j i} \gamma_{i j}, \\
\beta_{i j}+\beta_{j i}=\beta_{j k}+\beta_{k j}=: \beta, \\
\beta_{i j} \beta_{j k}=\left(\beta_{j k}-\beta_{j i}\right) \beta_{i k}=\left(\beta_{i j}-\beta_{k j}\right) \beta_{i k}, \\
\gamma_{i j} \gamma_{j k}=\left(\beta_{j i}-\beta_{j k}\right) \gamma_{i k}=\left(\beta_{k j}-\beta_{i j}\right) \gamma_{i k} .
\end{gathered}
$$

Proof. The Yang-Baxter system of equations $Y B(\hat{R})_{a b c}^{i j k}=0$ for a rime matrix is given in Appendix A. The subset (A.3)-(A.5) together with its image under the involution (A.2) reads

$$
\begin{aligned}
\alpha_{i j} \gamma_{i j}^{\prime}\left(\gamma_{i j}+\gamma_{j i}^{\prime}\right) & =0=\alpha_{i j} \gamma_{i j}\left(\gamma_{j i}+\gamma_{i j}^{\prime}\right) \\
\alpha_{i j}\left(\beta_{i j} \beta_{j i}+\gamma_{i j} \gamma_{i j}^{\prime}\right)=0 & =\alpha_{i j}\left(\beta_{i j} \beta_{j i}-\gamma_{i j} \gamma_{j i}\right), \\
\alpha_{i j} \gamma_{i j}^{\prime}\left(\alpha_{i j}+\beta_{j i}-\alpha_{i}\right)=0 & =\alpha_{i j} \gamma_{i j}\left(\alpha_{i j}+\beta_{j i}-\alpha_{j}\right), \\
\alpha_{i j} \gamma_{i j}^{\prime}\left(\alpha_{j i}+\beta_{i j}-\alpha_{i}\right)=0 & =\alpha_{i j} \gamma_{i j}\left(\alpha_{j i}+\beta_{i j}-\alpha_{j}\right) .
\end{aligned}
$$

These equations are implied by (and, in the strict rime situation, are equivalent to) the following system

$$
\begin{aligned}
\gamma_{i j}^{\prime}=-\gamma_{j i}, \quad \alpha_{i j}+\beta_{j i} & =\alpha_{i}, \quad \alpha_{j i}+\beta_{i j}=\alpha_{i}, \\
\beta_{i j} \beta_{j i} & =\gamma_{j i} \gamma_{i j} .
\end{aligned}
$$

One checks that other equations $Y B(\hat{R})_{a b c}^{i j k}=0$, for which two indices among $\{i, j, k\}$ are different, follow from (1.15) and (1.16). The last two equations from (1.15) imply $\alpha_{i}=\alpha_{j}$ for all $i$ and $j$. As an overall rescaling of a solution of the Yang-Baxter equation by a constant is again a solution of the YangBaxter equation, we can, without loss of generality, set it to one,

$$
\alpha_{i}=1
$$

Equations (1.15) and (1.17) yield the form (1.6) of the matrix $\hat{R}$ and equation (1.7). 
Using (1.15), we rewrite the subset (A.12)-(A.14) together with its image under the involution (A.2) in the form

$$
\begin{aligned}
\left(\beta_{i j}+\beta_{j i}\right. & \left.-\beta_{i k}-\beta_{k i}\right) \gamma_{i j} \gamma_{i k}=0 \\
\alpha_{i j}\left(\beta_{i j} \beta_{j k}+\beta_{i k} \beta_{j i}-\beta_{i k} \beta_{j k}\right) & =0=\alpha_{j i}\left(\beta_{j i} \beta_{k j}+\beta_{k i} \beta_{i j}-\beta_{k i} \beta_{k j}\right), \\
\alpha_{i j}\left(\gamma_{i j} \gamma_{j k}+\gamma_{i k}\left(\beta_{j k}-\beta_{j i}\right)\right) & =0=\alpha_{j i}\left(\gamma_{j i} \gamma_{k j}+\gamma_{k i}\left(\beta_{k j}-\beta_{i j}\right)\right) .
\end{aligned}
$$

These equations are implied by (and, in the strict rime situation, are equivalent to) equations (1.8), (1.9) and (1.10), respectively. One checks that other equations $Y B(\hat{R})_{a b c}^{i j k}=0$ with three different indices $\{i, j, k\}$ follow from the system (1.7)-(1.10). The proof is finished.

Lemma 1.1. The rime Yang-Baxter solution $R$ (1.6) is of Hecke type,

$$
\hat{R}^{2}=\beta \hat{R}+(1-\beta) \mathbb{1} \otimes \mathbb{1} .
$$

Moreover, when $\beta \neq 2, R$ is of $G L$-type: it has two eigenvalues 1 and $\beta-1$ with multiplicities $\frac{n(n+1)}{2}$ and $\frac{n(n-1)}{2}$, respectively. When $\beta=2$ the matrix $\hat{R}$ has a non-trivial Jordanian structure.

Proof. In view of the block structure of rime matrices it is enough to check the Hecke relation $(1.21)$ for one elementary $(4 \times 4)$ block which follows from (1.7) and (1.8). When $\beta \neq 2$ the multiplicities $m_{1}$ and $m_{\beta-1}$ are solutions of the system

$$
m_{1}+m_{\beta-1}=n^{2}, \quad m_{1}+(\beta-1) m_{\beta-1}=n+\frac{n(n-1)}{2} \beta(\equiv \operatorname{Tr} \hat{R}) .
$$

When $\beta=2$ the matrix $\hat{R}$ has only one eigenvalue 1 but $\hat{R} \neq \mathbb{1} \otimes \mathbb{1}$ due to (1.7) and (1.8).

Unitary solutions, $\hat{R}^{2}=\mathbb{1} \otimes \mathbb{1}$ are singled out by the value of the parameter $\beta=0$.

Lemma 1.2. A strict rime Yang-Baxter solution $R$ (1.6) can be brought to a rime matrix

$$
\hat{R}_{k l}^{i j}=\left(1-\beta_{j i}\right) \delta_{l}^{i} \delta_{k}^{j}+\beta_{i j} \delta_{k}^{i} \delta_{l}^{j}-\beta_{i j} \delta_{k}^{i} \delta_{l}^{i}+\beta_{j i} \delta_{k}^{j} \delta_{l}^{j},
$$

that is, to a solution (1.6) with $\gamma_{i j}=-\beta_{i j}$, by a change of basis. 
Proof. The strict rime condition $\alpha_{i j} \gamma_{i j} \neq 0$ implies $\beta_{i j} \beta_{j i} \neq 0$ in view of (1.7). Thus for a strict rime $R$-matrix all $\beta_{i j}$ and $\gamma_{i j}$ are non-vanishing. The ratio of equations (1.9) and (1.10) is well-defined and it follows from equations (1.7) and (1.8) that

$$
\frac{\gamma_{i j} \gamma_{j k}}{\beta_{i j} \beta_{j k}}=-\frac{\left(\beta_{j i}-\beta_{j k}\right) \gamma_{i k}}{\left(\beta_{j i}-\beta_{j k}\right) \beta_{i k}}=-\frac{\gamma_{i k}}{\beta_{i k}}, \quad \frac{\gamma_{i j} \gamma_{j i}}{\beta_{i j} \beta_{j i}}=1
$$

or

$$
\xi_{i j} \xi_{j k}=\xi_{i k}, \quad \xi_{i j} \xi_{j i}=1
$$

where $\xi_{i j}=-\frac{\gamma_{i j}}{\beta_{i j}}$. Equation (1.25) is solved by $\xi_{i j}=\frac{d_{i}}{d_{j}}$ with $d_{i} \neq 0, i=1$, $\ldots, n$, hence $\beta$ 's and $\gamma$ 's are related by

$$
\gamma_{i j}=-\frac{d_{i}}{d_{j}} \beta_{i j}
$$

A change of basis with a matrix $D$,

$$
\hat{R} \longmapsto(D \otimes D) \hat{R}\left(D^{-1} \otimes D^{-1}\right),
$$

where $D_{j}^{i}=d_{j} \delta_{j}^{i}$, transforms $R$ to the form (1.23).

Under the strict rime condition, the Yang-Baxter system of equations (see Appendix A) reduces to equations (1.8) and (1.9). However, the matrix (1.23), where the parameters $\beta_{i j}$ are subject to equations (1.8) and (1.9), is a solution of the Yang-Baxter equation without a strict rime assumption.

Remark. Right and left even quantum spaces are defined by, respectively,

$$
R_{k l}^{i j} x^{k} x^{l}=x^{i} x^{j}, \quad x_{j} x_{i} R_{k l}^{i j}=x_{l} x_{k}
$$

right and left odd quantum spaces are defined by, respectively,

$$
R_{k l}^{i j} \xi^{k} \xi^{l}=(\beta-1) \xi^{i} \xi^{j}, \quad \xi_{j} \xi_{i} R_{k l}^{i j}=(\beta-1) \xi_{l} \xi_{k} .
$$


Assume that $\beta \neq 2$. The left even space is classical ${ }^{1}$ as well as the right odd space

$$
\left[x_{i}, x_{j}\right]=0, \quad\left[\xi^{i}, \xi^{j}\right]_{+}=0,
$$

where $[$,$] and [,]_{+}$stand for the commutator and the anti-commutator. The relations for the right even space are

$$
\left[x^{i}, x^{j}\right]+\left(\beta_{i j} x^{i}+\beta_{j i} x^{j}\right)\left(x^{i}-x^{j}\right)=0
$$

the relations for the left odd space read

$$
\begin{gathered}
(2-\beta) \xi_{i}^{2}+\xi_{i} \rho+(1-\beta) \rho \xi_{i}=0, \\
{\left[\xi_{i}, \xi_{j}\right]_{+}-\beta_{i j} \xi_{i} \xi_{j}-\beta_{j i} \xi_{j} \xi_{i}=0, \quad i \neq j,}
\end{gathered}
$$

where $\rho=\sum_{j} \xi_{j}$.

\section{Rime Yang-Baxter solutions}

In this section we solve equations (1.8) and (1.9) thus obtaining explicitly rime Yang-Baxter solutions.

\section{$2.1 \quad$ Non-unitary rime $R$-matrices}

Proposition 2.1. The non-unitary strict rime Yang-Baxter solutions (1.23) with a parameter $\beta=\beta_{j i}+\beta_{i j} \neq 0$ are parameterized by a point $\phi \in \mathbb{P C}^{n}$ in a projective space, $\phi=\left(\phi_{1}: \phi_{2}: \ldots: \phi_{n}\right)$, such that $\phi_{i} \neq 0$ for all $i$ and $\phi_{i} \neq \phi_{j}$ for all $i$ and $j, i \neq j$. These solutions are given by

$$
\beta_{i j}=\frac{\beta \phi_{i}}{\phi_{i}-\phi_{j}}
$$

\footnotetext{
${ }^{1}$ Let $\hat{R}$ be a rime $R$-matrix (not necessarily strict). When $\beta \neq 2$, the following statement holds. If (i) the left even space is classical (which implies that $\gamma_{i j}^{\prime}=-\gamma_{j i}, \alpha_{i j}+\beta_{j i}=1$ and $\alpha_{i}=1$ in our normalization) and (ii) the $R$-matrix is Hecke (which implies that $\beta_{i j}+\beta_{j i}=\beta$ ), then the system of equations from Appendix A again reduces to (1.7), (1.9) and (1.10) as in the strict rime situation.
} 
Proof. Taking the ratio of the following pairs of equations from (1.9)

$$
\beta_{i j} \beta_{j k}=\left(\beta_{j k}-\beta_{j i}\right) \beta_{i k}, \quad \beta_{k j} \beta_{j i}=\left(\beta_{j i}-\beta_{j k}\right) \beta_{k i}
$$

we find that quantities $\eta_{i j}=-\beta_{i j} / \beta_{j i}$ verify equations

$$
\eta_{i j} \eta_{j k}=\eta_{i k}, \quad \eta_{i j} \eta_{j i}=1
$$

whose solutions are $\eta_{i j}=\phi_{i} / \phi_{j}$ for some constants $\phi_{i} \neq 0, i=1, \ldots, n$.

Substituting the relation $\beta_{j i}=-\frac{\phi_{j}}{\phi_{i}} \beta_{i j}$ into $\beta=\beta_{i j}+\beta_{j i}$, we obtain $\beta_{i j}-\frac{\phi_{j}}{\phi_{i}} \beta_{i j}=\beta$ which establishes (2.1).

Remark. There is a different parameterization, $\beta_{i j}=-\frac{\beta \phi_{j}}{\phi_{i}-\phi_{j}}$, of strict rime solutions; it is related to the parameterization $(2.1)$ by $\phi_{i} \longmapsto\left(\phi_{i}\right)^{-1}$.

A direct check shows that the condition $\phi_{i} \neq 0$ is not necessary: the formula (2.1) with $\phi_{i} \neq \phi_{j}$ for all $i$ and $j, i \neq j$, gives a rime solution of the Yang-Baxter equation. However when one of $\phi_{i}$ is 0 , the matrix (1.23) is no more strict.

\section{$2.2 \quad$ Unitary rime $R$-matrices}

For a unitary strict rime Yang-Baxter solution (1.23), $\hat{R}^{2}=\mathbb{1}$, we have $\beta=0$, so $\beta_{i j}=-\beta_{j i}$.

Proposition 2.2. The unitary strict rime Yang-Baxter solutions (1.23) are parameterized by a vector $\left(\mu_{1}, \ldots, \mu_{n}\right)$ such that $\mu_{i} \neq \mu_{j}$,

$$
\beta_{i j}=\frac{1}{\mu_{i}-\mu_{j}}
$$

Proof. Since $\beta_{i j}=-\beta_{j i}$ we can rewrite $\beta_{i j} \beta_{j k}=\left(\beta_{j k}-\beta_{j i}\right) \beta_{i k}$ as $\beta_{i j} \beta_{j k}=$ $\left(\beta_{i j}+\beta_{j k}\right) \beta_{i k}$ or

$$
\frac{1}{\beta_{i k}}=\frac{1}{\beta_{i j}}+\frac{1}{\beta_{j k}} .
$$


These equations are solved by

$$
\frac{1}{\beta_{i j}}=\mu_{i}-\mu_{j}
$$

which is equivalent to (2.4).

Remark. The unitary $R$-matrices of Proposition 2.2 can be obtained as a limit $\beta \rightarrow 0$ of the non-unitary $R$-matrices of Proposition 2.1. Indeed, for the following expansion of the parameters $\phi_{i}$ in the "small" parameter $\beta$,

$$
\phi_{i}=1+\beta \mu_{i}+o(\beta)
$$

the expression (2.1) has a limit (2.4),

$$
\beta_{i j}=\frac{\beta\left(1+\beta \mu_{i}+o(\beta)\right)}{\beta \mu_{i}-\beta \mu_{j}+o(\beta)} \stackrel{\beta \rightarrow 0}{\longrightarrow} \quad \beta_{i j}=\frac{1}{\mu_{i}-\mu_{j}} .
$$

\subsection{Properties}

1. Denote the $R$-matrix (1.23) with $\beta_{i j}$ as in $(2.1)$ by $\hat{R}(\vec{\phi})$. Let $\hat{R}_{21}=$ $P \hat{R}_{12} P$, where $P$ is the permutation operator. Then the following holds:

$$
\hat{R}_{21}(\vec{\phi})=F^{-1} \otimes F^{-1} R_{12}\left(\vec{\phi}^{-1}\right) F \otimes F
$$

where $F=\operatorname{diag}\left(\phi_{1}, \phi_{2}, \ldots, \phi_{n}\right)$ and $\vec{\phi}^{-1}$ is a vector with components $\phi_{i}^{-1}$.

Denote the $R$-matrix (1.23) with $\beta_{i j}$ as in $(2.4)$ by $\hat{R}(\vec{\mu})$. Then the following holds:

$$
\hat{R}_{21}(\vec{\mu})=\hat{R}_{12}(-\vec{\mu})
$$

2. The $R$-matrix (1.23) is skew invertible in the sense that there exists an operator $\hat{\Psi}_{R}$, which satisfies (see, e.g., [21])

$$
\operatorname{Tr}_{2}\left(\hat{R}_{12}\left(\hat{\Psi}_{R}\right)_{23}\right)=P_{13} .
$$

The matrices of the left and right quantum traces (that is, the left and right traces of the skew inverse $\left.\hat{\Psi}_{R}\right), \quad\left(Q_{R}\right)_{1}=\operatorname{Tr}_{2}\left(\left(\hat{\Psi}_{R}\right)_{12}\right)$ and 
$\left(\tilde{Q}_{R}\right)_{2}=\operatorname{Tr}_{1}\left(\left(\hat{\Psi}_{R}\right)_{12}\right)$ are given by the formulas

$$
\begin{gathered}
\left(Q_{R}\right)_{j}^{k}=-\beta_{j k} \prod_{l: l \neq k}\left(1-\beta_{j l}\right), \quad k \neq j, \text { and }\left(Q_{R}\right)_{j}^{j}=\prod_{l}\left(1-\beta_{j l}\right) ; \\
\left(\tilde{Q}_{R}\right)_{j}^{k}=\beta_{j k} \prod_{l: l \neq k}\left(1-\beta_{l j}\right), \quad k \neq j, \quad \text { and }\left(\tilde{Q}_{R}\right)_{j}^{j}=\prod_{l}\left(1-\beta_{l j}\right) .
\end{gathered}
$$

The matrices $Q_{R}$ and $\tilde{Q}_{R}$ satisfy $Q_{R} \tilde{Q}_{R}=(1-\beta)^{n-1} \mathbb{1}$.

For (2.1), one has $\operatorname{Spec} Q_{R}=\operatorname{Spec} \tilde{Q}_{R}=\left\{(1-\beta)^{a}, a=0, \ldots, n-1\right\}$. The eigenvector $w_{a}(\vec{\phi})$ of the matrix $Q_{R}$ with the eigenvalue $(1-\beta)^{n-1-a}$ coincides with the eigenvector of the matrix $\tilde{Q}_{R}$ with the eigenvalue $(1-\beta)^{a}$. One has $\left(w_{a}(\vec{\phi})\right)^{j}=e_{a}^{\hat{j}}(\vec{\phi})$, where $e_{i}^{\hat{j}}(\vec{\phi})$ is the $i$ th elementary symmetric function of $\left(\phi_{1}, \phi_{2}, \ldots, \phi_{n}\right)$ with $\phi_{j}$ omitted.

For (2.4), the Jordanian form of the matrix $Q_{R}$, as well as of $\tilde{Q}_{R}$, is nontrivial: it is a single block. In the basis $\left\{w_{i}(\vec{\mu})\right\}, i=0,1,2, \ldots, n-1$, where $\left(w_{i}(\vec{\mu})\right)^{j}=e_{i}^{\hat{j}}(\vec{\mu})$, one has

$$
Q_{R} w_{i}(\vec{\mu})=\sum_{s=0}^{i}\left(\begin{array}{c}
n-1-s \\
i-s
\end{array}\right) w_{s}(\vec{\mu})
$$

3. For an $R$-matrix $\hat{R}$, the group of invertible matrices $Y$ satisfying

$$
\hat{R}_{12} Y_{1} Y_{2}=Y_{1} Y_{2} \hat{R}_{12}
$$

form the invariance group $G_{R}$ of $\hat{R}$. The matrices $Q_{R}$ and $\tilde{Q}_{R}$ belong to the invariance group as well as the matrices proportional to the identity matrix. One can write down formulas for the group $G_{R}$ for a rime $R$-matrix (1.23) uniformly in terms of $\beta_{i j}$ as in (2.12) and (2.13) but the properties are different in the non-unitary and unitary cases and we describe them separately.

3a. The invariance group $G_{R(\vec{\phi})}$ for the $R$-matrix $\hat{R}(\vec{\phi})$ is 2-parametric. It consists of matrices $Y(u, v), u, v \neq 0$, where

$$
\begin{aligned}
Y(u, v)_{j}^{j} & =\prod_{l: l \neq j} \frac{u \phi_{j}-v \phi_{l}}{\phi_{j}-\phi_{l}} \text { and } \\
Y(u, v)_{j}^{i} & =\frac{(u-v) \phi_{j}}{\phi_{j}-\phi_{i}} \prod_{l: l \neq i, j} \frac{u \phi_{j}-v \phi_{l}}{\phi_{j}-\phi_{l}}, \quad i \neq j .
\end{aligned}
$$


One has

$$
Q_{R(\vec{\phi})}=Y(1-\beta, 1), \quad \tilde{Q}_{R(\vec{\phi})}=Y(1,1-\beta)
$$

The composition law is the component-wise multiplication of the parameters $\{u, v\}$,

$$
Y\left(u_{1}, v_{1}\right) Y\left(u_{2}, v_{2}\right)=Y\left(u_{1} u_{2}, v_{1} v_{2}\right)
$$

The point $u=v=1$ corresponds to the identity matrix, $Y(1,1)=\mathbb{1}$; the determinant of $Y(u, v)$ is $(u v)^{n(n-1) / 2} ; u=v$ corresponds to global rescalings; the connected component of unity of the subgroup $\mathrm{SG}_{R(\vec{\phi})}$ consisting of matrices with determinant 1 is $u v=1$; the generator $\eta$ of the connected component of unity of the subgroup $\mathrm{SG}_{R(\vec{\phi})}$ is traceless and reads

$$
\eta_{j}^{i}=\frac{\phi_{j}}{\phi_{j}-\phi_{i}}, \quad i \neq j \quad \text { and } \quad \eta_{j}^{j}=-\frac{n-1}{2}+\sum_{l: l \neq j} \frac{1}{\phi_{j}-\phi_{l}}
$$

3b. For the $R$-matrix $\hat{R}(\vec{\mu})$, the group $\mathrm{SG}_{R(\vec{\mu})}$, consisting of matrices with determinant 1 is 1 -parametric as well. It is formed by matrices $Y^{(0)}(a)$, where

$$
\begin{aligned}
Y^{(0)}(a)_{j}^{j} & =\prod_{l: l \neq j}\left(1+\frac{a}{\mu_{j}-\mu_{l}}\right) \text { and } \\
Y^{(0)}(a)_{j}^{i} & =\frac{a}{\mu_{j}-\mu_{i}} \prod_{l: l \neq i, j}\left(1+\frac{a}{\mu_{j}-\mu_{l}}\right), i \neq j .
\end{aligned}
$$

The expression (2.20) can be obtained by taking a limit of (2.16), similarly to (2.8) and letting additionally $u=1+a \beta / 2+o(\beta)$ and $v=1-a \beta / 2+o(\beta)$.

One has

$$
Q_{R(\vec{\mu})}=Y^{(0)}(-1), \quad \tilde{Q}_{R(\vec{\mu})}=Y^{(0)}(1)
$$

The composition law is $Y^{(0)}\left(a_{1}\right) Y^{(0)}\left(a_{2}\right)=Y^{(0)}\left(a_{1}+a_{2}\right)$.

The point $a=0$ in $(2.20)$ corresponds to the identity matrix, $Y^{(0)}(0)=\mathbb{1}$; the generator $\eta^{(0)}$ of the invariance group $\mathrm{SG}_{R(\vec{\mu})}$ is

$$
\left(\eta^{(0)}\right)_{j}^{i}=\frac{1}{\mu_{j}-\mu_{i}}, \quad i \neq j \quad \text { and } \quad\left(\eta^{(0)}\right)_{j}^{j}=\sum_{l: l \neq j} \frac{1}{\mu_{j}-\mu_{l}}
$$




\section{Rime and Cremmer-Gervais $R$-matrices}

The Cremmer-Gervais $R$-matrix arises in the exchange relations of the chiral vertex operators in the non-linearly $W$-extended Virasoro algebra [6]. The Cremmer-Gervais solution [6] of the Yang-Baxter equation in its general two-parametric form reads (see, e.g., [17]; we use a rescaled matrix with eigenvalues 1 and $-q^{-2}$ )

$$
\begin{aligned}
\left(\hat{R}_{\mathrm{CG}, p}\right)_{k l}^{i j}= & q^{-2 \theta_{i j}} p^{i-j} \delta_{l}^{i} \delta_{k}^{j}+\left(1-q^{-2}\right) \sum_{s: i \leq s<j} p^{i-s} \delta_{k}^{s} \delta_{l}^{i+j-s} \\
& -\left(1-q^{-2}\right) \sum_{s: j<s<i} p^{i-s} \delta_{k}^{s} \delta_{l}^{i+j-s}
\end{aligned}
$$

where $\theta_{i j}$ is the step function $\left(\theta_{i j}=1\right.$ when $i>j$ and $\theta_{i j}=0$ when $\left.i \leq j\right)$.

The parameter value $p=q^{2 / n}$ specifies the $\mathrm{SL}(n)$ Cremmer-Gervais $R$-matrix (its diagonal twist being the $\mathrm{GL}(n)$ solution (3.1)). The CremmerGervais solution is a non-diagonal twist of the standard Drinfeld-Jimbo solution $[9,18]$.

Let $\hat{R}_{\mathrm{CG}}:=\hat{R}_{\mathrm{CG}, 1}$, that is, the solution (3.1) with $p=1$. The matrix

$$
D(p)_{j}^{i}=\delta_{j}^{i} p^{i-1}
$$

with arbitrary $p$ satisfies $\left(\hat{R}_{\mathrm{CG}}\right)_{12} D(p)_{1} D(p)_{2}=D(p)_{1} D(p)_{2}\left(\hat{R}_{\mathrm{CG}}\right)_{12}$. It was observed in [10] that if $\hat{R}_{12} D_{1} D_{2}=D_{1} D_{2} \hat{R}_{12}$ for some $R$-matrix $\hat{R}$ and operator $D$ then $D_{1} \hat{R}_{12} D_{1}^{-1}$ is again an $R$-matrix (this operation was also used in [15] to partially change the statistics of ghosts in the super-symmetric situation). The two-parametric matrix $\hat{R}_{\mathrm{CG}, p}(3.1)$ can be obtained from the Cremmer-Gervais matrix $\hat{R}_{C G}$ by this operation as well,

$$
\left(\hat{R}_{\mathrm{CG}, p}\right)_{12}=D(p)_{1}\left(\hat{R}_{\mathrm{CG}}\right)_{12} D(p)_{1}^{-1} .
$$

Let $\hat{R}$ be the non-unitary rime matrix from Proposition 2.1 with $\phi_{i} \neq \phi_{j}$.

Proposition 3.1. The matrix $\hat{R}$ transforms into the Cremmer-Gervais solution $\hat{R}_{C G}$

$$
\hat{R}=(X \otimes X) \hat{R}_{\mathrm{CG}}\left(X^{-1} \otimes X^{-1}\right)
$$

by a change of basis with the invertible matrix

$$
X_{j}^{k}=e_{j-1}\left(\phi_{1}, \ldots, \hat{\phi}_{k}, \ldots, \phi_{n}\right)=: e_{j-1}^{\hat{k}}
$$


whose inverse is

$$
\left(X^{-1}\right)_{i}^{j}=\frac{(-1)^{j-1} \phi_{i}^{n-j}}{\prod_{k: k \neq i}\left(\phi_{i}-\phi_{k}\right)} .
$$

Here the hat over $\phi_{j}$ means that this entry is omitted in the expression and $e_{i}$ are the elementary symmetric polynomials $e_{i}\left(x_{1}, \ldots, x_{N}\right)=\sum_{s_{1}<\ldots<s_{i}}$ $x_{s_{1}} x_{s_{2}} \ldots x_{s_{i}}$. The projective parameters $\left(\phi_{1}: \phi_{2}: \ldots: \phi_{n}\right)$ are removed by the transformation $X$ and the only essential parameter $\beta$ in $\hat{R}$ is related to the parameter $q$ in $\hat{R}_{\mathrm{CG}}$ by

$$
q^{-2}=1-\beta
$$

Proof. Due to the Lagrange interpolation formula, the matrix, inverse to the Vandermonde matrix

$$
\left\|V_{k}^{j}\right\|_{j, k=1}^{n}=\phi_{k}^{n-j} \quad \text { is } \quad\left(V^{-1}\right)_{j}^{k}=\frac{(-1)^{j-1} e_{j-1}^{\hat{k}}}{\prod_{l: l \neq k}\left(\phi_{k}-\phi_{l}\right)} .
$$

The matrix $X$ (3.5) has the form $X=D V^{-1} d$, where $D_{k}^{m}=\delta_{k}^{m} \prod_{l: l \neq k}$ $\left(\phi_{k}-\phi_{l}\right)$ and $d_{j}^{i}=(-1)^{j-1} \delta_{j}^{i}$ are diagonal $n \times n$ matrices. Thus, its inverse is $X^{-1}=d^{-1} V D^{-1}$, which establishes (3.6).

We now prove the matrix identity (3.4) in the form

$$
\hat{R}(X \otimes X)=(X \otimes X) \hat{R}_{\mathrm{CG}} .
$$

The substitution of the explicit form of the rime matrix $\hat{R}$ (1.23) with $\beta_{i j}=\beta \phi_{i} /\left(\phi_{i}-\phi_{j}\right)$ and $\hat{R}_{\mathrm{CG}}$ (3.1) reduces (3.9) to a set of relations between the symmetrical polynomials $e_{k-1}^{\hat{a}}$

$$
\sum_{a, b} \hat{R}_{a b}^{i j} e_{k-1}^{\hat{a}} e_{l-1}^{\hat{b}}=\sum_{a, b} e_{a-1}^{\hat{i}} e_{b-1}^{\hat{j}}\left(\hat{R}_{\mathrm{CG}}\right)_{k l}^{a b} .
$$

There are two subcases: (i) $i=j$ and (ii) $i \neq j$.

(i) The left-hand side of equation (3.10) with $i=j$ is just $e_{k-1}^{\hat{i}} e_{l-1}^{\hat{i}}$ due to the rime condition. Equation (3.10) is satisfied because of the symmetry relation $\left(\hat{R}_{\mathrm{CG}}\right)_{k l}^{a b}=\delta_{k}^{a} \delta_{l}^{b}+\delta_{l}^{a} \delta_{k}^{b}-\left(\hat{R}_{\mathrm{CG}}\right)_{k l}^{b a}$. 
(ii) For $i \neq j$ equation (3.10), where $q^{-2}=1-\beta$, reduces, after some algebraic manipulations, to

$$
\begin{aligned}
& \frac{1}{\phi_{i}-\phi_{j}}\left(\phi_{i} e_{k-1}^{\hat{i}}-\phi_{j} e_{k-1}^{\hat{j}}\right)\left(e_{l-1}^{\hat{i}}-e_{l-1}^{\hat{j}}\right) \\
& =\sum_{s: s \geq \max (1, k-l+2)}\left(e_{l+s-2}^{\hat{i}} e_{k-s}^{\hat{j}}-e_{l+s-2}^{\hat{j}} e_{k-s}^{\hat{i}}\right), \quad 1 \leq i, j, k, l \leq n .
\end{aligned}
$$

In fact, the sum in the right-hand side goes till $s=\min (k, n+1-l)$ since $e_{r}^{\hat{j}}=0$ when $r \geq n-1$; moreover, we can start the summation from $s=1$ because when $1<k-l+2$ the sum for $1 \leq s \leq k-l+1$ is anti-symmetric under $s \longleftrightarrow k-l+2-s$ and thus vanishes.

To prove (3.11) we write $e_{r}=e_{r}^{\hat{i}}+\phi_{i} e_{r-1}^{\hat{i}}$; therefore $e_{r}^{\hat{i}}=e_{r}^{\hat{i} \hat{j}}+\phi_{j} e_{r-1}^{\hat{i} \hat{j}}$ and $e_{r}^{\hat{j}}=e_{r}^{\hat{i} \hat{j}}+\phi_{i} e_{r-1}^{\hat{i} \hat{j}}$ and equation (3.11) becomes

$$
-\left(\phi_{i}-\phi_{j}\right) e_{k-1}^{\hat{i} \hat{j}} e_{l-2}^{\hat{i} \hat{j}}=\left(\phi_{i}-\phi_{j}\right) \sum_{s \geq 1}\left(e_{l+s-2}^{\hat{i} \hat{j}} e_{k-s-1}^{\hat{i} \hat{j}}-e_{l+s-3}^{\hat{i} \hat{j}} e_{k-s}^{\hat{i} j}\right) .
$$

The sum in the right-hand side telescopes to the value of $\left(-e_{l+s-3}^{\hat{i} \hat{j}} e_{k-s}^{\hat{i} \hat{j}}\right)$ at $s=1$, that is, to $\left(-e_{k-1}^{\hat{i} \hat{j}} e_{l-2}^{\hat{i} \hat{j}}\right)$. The proof is complete.

It should be noted that the matrix $X=X(\vec{\phi})$ does not depend on $q$. The change of the basis with the matrix $X\left(\vec{\phi}^{\prime}\right) X(\vec{\phi})^{-1}$ transforms the $R$-matrix $\hat{R}(\vec{\phi})$ to $\hat{R}\left(\vec{\phi}^{\prime}\right)$. We have

$$
\left(X\left(\overrightarrow{\phi^{\prime}}\right) X(\vec{\phi})^{-1}\right)_{j}^{i}=\frac{1}{\phi_{j}-\phi_{i}^{\prime}} \frac{\prod_{k}\left(\phi_{j}-\phi_{k}^{\prime}\right)}{\prod_{l: l \neq j}\left(\phi_{j}-\phi_{l}\right)} .
$$

The structure of the matrices $X$ and $X^{-1}$ shows that when the dimension is infinite, the $R$-matrices $\hat{R}_{\mathrm{CG}, 1}$ and $\hat{R}(\vec{\phi})$ (as well as the $R$-matrices $\hat{R}(\vec{\phi})$ and $\hat{R}\left(\vec{\phi}^{\prime}\right)$ for different $\phi$ and $\left.\phi^{\prime}\right)$ are in general not equivalent.

The right even quantum plane for the Cremmer-Gervais matrix $\hat{R}_{\mathrm{CG}, 1}$ is defined by the following equations:

$$
y^{i} y^{j}=q^{2} y^{j} y^{i}+\left(q^{2}-1\right)\left(y^{i+1} y^{j-1}+\ldots+y^{j-1} y^{i+1}\right), \quad i<j .
$$


If $i+1<j-1$, one uses the formula (3.14) recursively to get the ordering relations.

The change of basis with the matrix $X$,

$$
x^{i}=\sum_{j=1}^{n} e_{j-1}^{\hat{i}} y^{j},
$$

transformes the quantum plane (3.14) into the rime quantum plane (1.31) exhibiting coordinate two-dimensional subplanes. The change of basis (3.15) can be written in terms of a "generating function": let

$$
G:=\sum_{j} e_{j}\left(\phi_{1}, \ldots, \phi_{n}\right) y^{j} .
$$

Then

$$
x^{i}=\frac{\partial G}{\partial \phi_{i}} .
$$

Remark. The standard Drinfeld-Jimbo $R$-matrix admits, for a certain choice of multi-parameters, a different rime form. The relations $u^{i} v^{j}=$ $\left(\hat{R}_{c}\right)_{k l}^{i j} v^{k} u^{l}$ for this choice are

$$
\begin{aligned}
& u^{i} v^{i}=v^{i} u^{i}, \\
& u^{i} v^{j}=v^{j} u^{i}+\left(1-q^{-2}\right) v^{i} u^{j}, \quad i<j, \\
& u^{i} v^{j}=q^{-2} v^{j} u^{i}, \quad i>j .
\end{aligned}
$$

The left even space for this $R$-matrix is classical.

The change of variables with the matrix $\tilde{X}_{j}^{i}=1-\theta_{j i}$,

$$
U^{i}:=u^{1}+u^{2}+\ldots+u^{i}, \quad V^{i}:=v^{1}+v^{2}+\cdots+v^{i},
$$

transforms the relations (3.18) into

$$
\begin{aligned}
U^{i} V^{i} & =V^{i} U^{i}, \\
U^{i} V^{j} & =V^{j} U^{i}+\left(1-q^{-2}\right) V^{i} U^{j}-\left(1-q^{-2}\right) V^{i} U^{i}, \quad i<j, \\
U^{i} V^{j} & =q^{-2} V^{j} U^{i}+\left(1-q^{-2}\right) V^{j} U^{j}, \quad i>j .
\end{aligned}
$$

The matrix $X$, defined by equation (3.5), degenerates if $\phi_{i}=\phi_{j}$ for some $i$ and $j$. Interestingly, the $R$-matrix $X \otimes X \hat{R}_{c} X^{-1} \otimes X^{-1}$ admits limits 
$\lim _{\phi_{\sigma(2)} \rightarrow 0} \lim _{\phi_{\sigma(3)} \rightarrow 0} \ldots \lim _{\phi_{\sigma(n)} \rightarrow 0}$ for an arbitrary permutation $\sigma \in S_{n}$ and the result is always rime. In particular,

$$
\tilde{X} \otimes \tilde{X} \hat{R}_{c} \tilde{X}^{-1} \otimes \tilde{X}^{-1}=\lim _{\phi_{2} \rightarrow 0} \lim _{\phi_{3} \rightarrow 0} \ldots \lim _{\phi_{n} \rightarrow 0} X \otimes X \hat{R}_{c} X^{-1} \otimes X^{-1} .
$$

\section{Classical rime $r$-matrices}

The classical limit of an $R$-matrix is a classical r-matrix, a solution of the classical Yang-Baxter (cYB) equation

$$
\left[r_{12}, r_{13}\right]+\left[r_{12}, r_{23}\right]+\left[r_{13}, r_{23}\right]=0 .
$$

We are going to show that the classical limits of the rime $R$-matrices from Section 2 are equivalent to the Cremmer-Gervais $r$-matrices in the non-skew-symmetric case and to the "boundary" $r$-matrix of Gerstenhaber and Giaquinto [14] (see also [4]; this $r$-matrix is attributed to A. G. Elashvili there) in the skew-symmetric case. Similar equivalences appeared in the study of the gauge transformations of the dynamical $r$-matrices in the Calogero-Moser model $[12,13]^{2}$

In the sequel we use the following conventions. An $R$-matrix acts in a space $V \otimes V$. A basis of $V$ is $\left\{e_{i}\right\}$ (labeled by a lower index); an operator $A$ in $V$ has matrix coefficients $A_{i}^{j}, A\left(e_{i}\right)=A_{i}^{j} e_{j}$, so for a vector $\vec{v}=v^{i} e_{i}$ one has $(A \vec{v})^{i}=A_{j}^{i} \vec{v}^{j}$; the matrix units are $e_{j}^{i}, e_{j}^{i}\left(e_{k}\right)=\delta_{k}^{i} e_{j}$, so the multiplication rule is $e_{j}^{i} e_{l}^{k}=\delta_{l}^{i} e_{j}^{k} ; e_{\alpha_{i}}$ are the $\mathfrak{s l}(n)$ simple positive root elements, $e_{\alpha_{i}}=e_{i}^{i+1}$; $P$ is the permutation operator, $P\left(e_{i} \otimes e_{j}\right)=e_{j} \otimes e_{i}$, so $P\left(e_{j}^{i} \otimes e_{l}^{k}\right)=e_{l}^{i} \otimes e_{j}^{k}$ and $(P B)_{i j}^{k l}=B_{i j}^{l k}$ for an operator $B$ in $V \otimes V$ having matrix coefficients $B_{i j}^{k l}, B\left(e_{i} \otimes e_{j}\right)=B_{i j}^{k l} e_{k} \otimes e_{l}$.

\subsection{Non-skew-symmetric case}

Proposition 4.1. The non-unitary rime $R$-matrix (Proposition 2.1) is a quantization of the non-skew-symmetric r-matrix

$$
r=\sum_{i, j: i \neq j} \frac{\phi_{i}}{\phi_{i}-\phi_{j}}\left(e_{j}^{i} \otimes e_{i}^{j}-e_{i}^{i} \otimes e_{j}^{j}+e_{i}^{i} \wedge e_{j}^{i}\right),
$$

\footnotetext{
${ }^{2}$ We thank László Fehér for drawing our attention to references [12,13].
} 
where $x \wedge y:=x \otimes y-y \otimes x$. The change of basis with the matrix $X_{k}^{j}=e_{k-1}\left(\phi_{1}, \ldots, \hat{\phi}_{j}, \ldots, \phi_{n}\right)$ transforms $r$ into the parameter-free $c Y B$ solution $r_{\mathrm{CG}}$

$$
r_{\mathrm{CG}}=\sum_{i, j: i<j} \sum_{s=1}^{j-i}\left(e_{j}^{i+s-1} \otimes e_{i}^{j-s+1}-e_{i}^{i+s-1} \otimes e_{j}^{j-s+1}\right) .
$$

Proof. The coefficients $\beta_{i j}(2.1)$ are linear in the deformation parameter $\beta$ ( $\beta=0$ is the classical point). Hence

$$
R=\mathbb{1} \otimes \mathbb{1}+\beta r
$$

where $R=P \hat{R}$ and $r$ is given by (4.2).

The matrix $R_{\mathrm{CG}}-\mathbb{1} \otimes \mathbb{1}$, where $R_{\mathrm{CG}}=P \hat{R}_{\mathrm{CG}}$, is linear with respect to the parameter $\beta=1-q^{-2}$ as well,

$$
R_{\mathrm{CG}}=\mathbb{1} \otimes \mathbb{1}+\beta r_{\mathrm{CG}}
$$

thus the formula (3.4) implies $r=(X \otimes X) r_{\mathrm{CG}}\left(X^{-1} \otimes X^{-1}\right)$.

We mentioned two ways of obtaining the numerical two-parametric $R$-matrix $\left(\hat{R}_{\mathrm{CG}, p}\right)$ from the $R$-matrix $\left(\hat{R}_{\mathrm{CG}, 1}\right)$ : by a diagonal twist and by the operation (3.3). There is one more way which consists of changing the representation. We shall illustrate it on the example of the classical GL $r$-matrix (4.3). A change of representation of the Lie algebra GL,

$$
e_{j}^{i} \mapsto e_{j}^{i}+c \delta_{j}^{i} \mathbb{1}
$$

where $c$ is a constant, produces the following effect on the $r$-matrix (4.3):

$$
r_{\mathrm{CG}} \mapsto r_{\mathrm{CG}}+c(\eta \otimes \mathbb{1}-\mathbb{1} \otimes \eta-(n-1) \mathbb{1} \otimes \mathbb{1}),
$$

where $n=\operatorname{dim} V$ and

$$
\eta=-\frac{n(n+1)}{2} \mathbb{1}+\sum j e_{j}^{j}, \quad \operatorname{tr} \eta=0 .
$$


The classical version of the operation (3.3) is as follows. Let $\eta$ be an arbitrary generator of the invariance group of an $r$-matrix $r$,

$$
\left[r, \eta_{1}+\eta_{2}\right]=0
$$

Then the operator

$$
r_{(c)}=r+c\left(\eta_{1}-\eta_{2}\right),
$$

where $c$ is a constant, is again a classical $r$-matrix (a solution of the cYBe).

The operator $\eta$ in (4.8) is, up to a scale, the unique traceless generator of the invariance group (see (3.2)) of the $r$-matrix (4.3). Thus, the representation change and the operation (4.10) give the same family of $r$-matrices (up to an addition of a multiple of the identity operator, which does not violate the $\mathrm{cYBe})$.

\subsection{BD triples}

Each block in the strict rime classical $r$-matrix (4.2) looks even more "rimed",

$$
\left(\begin{array}{cccc}
0 & 0 & 0 & 0 \\
\beta_{12}^{\prime} & -\beta_{12}^{\prime} & \beta_{21}^{\prime} & -\beta_{21}^{\prime} \\
-\beta_{12}^{\prime} & \beta_{12}^{\prime} & -\beta_{21}^{\prime} & \beta_{21}^{\prime} \\
0 & 0 & 0 & 0
\end{array}\right)
$$

where $\beta_{i j}^{\prime}=\beta_{i j} / \beta=\phi_{i} /\left(\phi_{i}-\phi_{j}\right)$. The multiplication from the left by $P$ acts on each block as a permutation of the second and third lines, so the rime $r$-matrix (4.11) enjoys the symmetry $\operatorname{Pr}=-r$. We shall now discuss this symmetry property in the context of Belavin-Drinfeld triples.

In [3] Belavin and Drinfeld gave, for a simple Lie algebra $\mathfrak{g}$, a description of non-unitary (non-skew-symmetric) cYB solutions $r \in \mathfrak{g} \otimes \mathfrak{g}$, satisfying $r_{12}+r_{21}=t$, where $t \in \mathfrak{g} \otimes \mathfrak{g}$ is the $\mathfrak{g}$-invariant element. The non-unitary solutions are put into correspondence with combinatorial objects called Belavin-Drinfeld triples (BD-triples for short). The BD triple $\left(\Pi_{1}, \Pi_{2}, \tau\right)$ for a simple Lie algebra $\mathfrak{g}$ consists of the following data: $\Pi_{1}, \Pi_{2}$ are subsets of the set of simple positive roots $\Pi$ of the algebra $\mathfrak{g}$ and $\tau$ is an invertible mapping: $\Pi_{1} \rightarrow \Pi_{2}$ such that $\left\langle\tau(\rho), \tau\left(\rho^{\prime}\right)\right\rangle=\left\langle\rho, \rho^{\prime}\right\rangle$ for any $\rho, \rho^{\prime} \in \Pi_{1}$ and $\tau^{k}(\rho) \neq \rho$ for any $\rho \in \Pi_{1}$ and any natural $k$ for which $\tau^{k}(\rho)$ is defined. 
The $r$-matrix for a triple $\left(\Pi_{1}, \Pi_{2}, \tau\right)$ has the form

$$
r=r_{0}+\sum_{\alpha \in \Delta_{+}} e_{-\alpha} \otimes e_{\alpha}+\sum_{\alpha, \beta \in \Delta_{+}: \alpha<\beta} e_{-\alpha} \wedge e_{\beta},
$$

where $<$ is a partial order on the set of positive roots $\Delta_{+}$defined by the rule: $\alpha<\beta$ for $\alpha, \beta \in \Delta_{+}$if there exists a natural $k$ such that $\tau^{k}(\alpha)=\beta$. The part $r_{0}$ belongs to $\mathfrak{h} \otimes \mathfrak{h}$, where $\mathfrak{h}$ is the Cartan subalgebra of $\mathfrak{g} ; r_{0}$ contains continuous "multiparameters", which satisfy

$$
(\tau(\alpha) \otimes \mathrm{id}+\mathrm{id} \otimes \alpha)\left(r_{0}\right)=0 \quad \text { for all } \alpha \in \Pi_{1} .
$$

We are dealing with matrix solutions $r$ of the cYB equation, $r \in \mathfrak{g l}(n) \otimes \mathfrak{g l}(n)$, so $r_{12}+r_{21}$ can be a linear combination of $P$ and $\mathbb{1} \otimes \mathbb{1}$.

Let $\Pi=\left\{\alpha_{1}, \ldots, \alpha_{n-1}\right\}$ be the set of the positive simple roots for the Lie algebra $\mathfrak{s l}(n)$.

There are two Cremmer-Gervais BD triples, $\mathfrak{T}_{+}$and $\mathfrak{T}_{-}$. For the Cremmer-Gervais BD triple $\mathfrak{T}_{+}, \Pi_{1}=\left\{\alpha_{1}, \alpha_{2}, \ldots, \alpha_{n-2}\right\}, \Pi_{2}=\left\{\alpha_{2}, \alpha_{3}, \ldots\right.$, $\left.\alpha_{n-1}\right\}$ and $\tau\left(\alpha_{i}\right)=\alpha_{i+1}$. The data $\left(\Pi_{1}, \Pi_{2}, \tau\right)$ is encoded in the graph

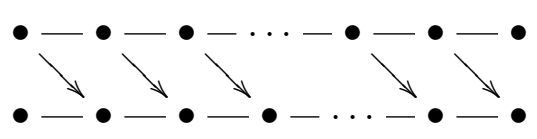

The triple $\mathfrak{T}_{-}$can be obtained from the triple $\mathfrak{T}_{+}$either by setting $\Pi_{1}^{\prime}=\Pi_{2}, \Pi_{2}^{\prime}=\Pi_{1}$ and $\tau^{\prime}=\tau^{-1}$ or by applying the outer automorphism of the underlying $A_{n-1}$ Dynkin diagram; the graph corresponding to the triple $\mathfrak{T}_{-}$is

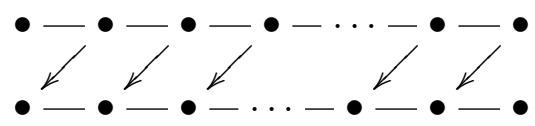

The $r$-matrix (4.3) corresponds to the triple (4.14) for a certain choice of the multiparameters. Here is the $r$-matrix $r^{\prime}$ corresponding to the triple (4.15)

$$
r_{\mathrm{CG}}^{\prime}=\sum_{i, j: i<j} \sum_{s=1}^{j-i}\left(e_{j-s+1}^{i} \otimes e_{i+s-1}^{j}-e_{j-s+1}^{j} \otimes e_{i+s-1}^{i}\right)
$$

for a certain choice of the multiparameters, for which it satisfies $r^{\prime} P=-r^{\prime}$. 
For the $r$-matrices (4.3) and (4.16), one has $r_{12}+r_{21}=P-\mathbb{1} \otimes \mathbb{1}$. The Cartan part of the $r$-matrices (4.3) and (4.16) are

$$
r_{0}=-\sum_{i, j: i<j} e_{i}^{i} \otimes e_{j}^{j}, \quad r_{0}^{\prime}=-\sum_{i, j: i<j} e_{j}^{j} \otimes e_{i}^{i} .
$$

The following lemma shows that a classical $r$-matrix $r$ for a triple $\mathfrak{T}$ can have a symmetry with respect to the multiplication by $P$ from one side if and only if all segments (connected components) of $\Pi_{1}$ are mapped by $\tau$ according to either (4.14) or (4.15).

Lemma 4.1. A non-skew-symmetric classical $r$-matrix with a Belavin-Drinfeld data $\left(\Pi_{1}, \Pi_{2}, \tau\right)$ can satisfy $\operatorname{Pr}=-r \quad$ (respectively, $r P=-r)$ for a certain choice of the multiparameters if and only if $\tau\left(\alpha_{i}\right)=\alpha_{i+1}$ (respectively, $\left.\tau\left(\alpha_{i}\right)=\alpha_{i-1}\right)$ for all $i \in \Pi_{1}$.

Proof. Assume that $\tau\left(\alpha_{m}\right)=\alpha_{m+k}$ for some natural $k, k \geq 1$. Then $r$ contains the term $e_{m+k+1}^{m+k} \wedge e_{m}^{m+1}$ with the coefficient 1. Such $r$-matrix cannot satisfy $r P=-r$ for if $r P=-r$ then $r$ contains the term $e_{m+k+1}^{m+1} \wedge$ $e_{m}^{m+k}$ with the coefficient $(-1)$ but the coefficient in $e_{-\alpha} \wedge e_{\beta}$ is 1 in the formula (4.12).

If $\operatorname{Pr}=-r$ then $r$ should contain also the term $e_{m+k+1}^{m+1} \wedge e_{m}^{m+k}$. It then follows that

(i) the Lie subalgebra generated by $\Pi_{1}$ contains $e_{m}^{m+k}$, therefore the interval $\left[\alpha_{m}, \alpha_{m+1}, \ldots, \alpha_{m+k-1}\right]$ is contained in $\Pi_{1}$;

(ii) the Lie subalgebra generated by $\Pi_{2}$ contains $e_{m+k+1}^{m+1}$, therefore the interval $\left[\alpha_{m+1}, \alpha_{m+2}, \ldots, \alpha_{m+k}\right]$ is contained in $\Pi_{2}$;

(iii) the image of the interval $\left[\alpha_{m}, \alpha_{m+1}, \ldots, \alpha_{m+k-1}\right]$ under $\tau$ is the interval $\left[\alpha_{m+1}, \alpha_{m+2}, \ldots, \alpha_{m+k}\right]$.

This implies that the interval $\left[\alpha_{m+1}, \alpha_{m+2}, \ldots, \alpha_{m+k-1}\right]$ is $\tau$-invariant (since $\tau\left(\alpha_{m}\right)=\alpha_{m+k}$ ), which contradicts to the nilpotency of $\tau$ unless this interval is empty, that is, $k=1$.

Similarly, $r P=-r$ is possible only if $\tau\left(\alpha_{i}\right)=\alpha_{i-1}$ for all $i \in \Pi_{1}$.

It is left to show that when $\tau\left(\alpha_{i}\right)=\alpha_{i+1}$ (respectively, $\tau\left(\alpha_{i}\right)=\alpha_{i-1}$ ) for all $i \in \Pi_{1}$ the multiparameters can indeed be adjusted to fulfill $\operatorname{Pr}=-r$ (respectively, $r P=-r$ ). We leave it as an exercise for the reader to check that with the assignment (4.17) for $r$ (respectively, for $r^{\prime}$ ) the compatibility condition (4.13) is verified. The proof is finished. 
Remark. Two extreme BD triples can be rimed, the empty (DrinfeldJimbo) one and the "maximal" Cremmer-Gervais one. However, not every triple can be rimed: already the triple

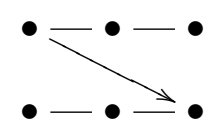

provides a counterexample. We outline a computer-aided proof in Appendix C.

\subsection{Skew-symmetric case}

A skew-symmetric classical $r$-matrix $r \in \mathfrak{g} \wedge \mathfrak{g}$ is canonically associated with a quasi-Frobenius Lie subalgebra $(\mathfrak{f}, \omega)$ of $\mathfrak{g}$ (see, e.g., [24]). A Lie algebra $\mathfrak{f}$ which admits a non-degenerate 2-cocycle $\omega$ is called quasi-Frobenius; it is Frobenius if $\omega$ is a coboundary, i.e., $\omega(X, Y)=\lambda([X, Y])$ for some $\lambda \in \mathfrak{f}^{*}$.

We describe now the skew-symmetric $r$-matrix arising in the classical limit of the unitary rime $R$-matrix from Proposition 2.2.

Proposition 4.2. The unitary rime R-matrix (Proposition 2.2) is a quantization of the skew-symmetric r-matrix

$$
r=\sum_{i, j: i<j} \frac{1}{\mu_{i}-\mu_{j}}\left(e_{j}^{i}-e_{j}^{j}\right) \wedge\left(e_{i}^{j}-e_{i}^{i}\right) \in \mathfrak{g l}(n) \wedge \mathfrak{g l}(n) .
$$

This skew-symmetric classical $r$-matrix corresponds to a Frobenius Lie algebra $\left(\mathfrak{g}_{0}(n), \delta \lambda_{n}\right)$ spanned by the generators $Z_{j}^{i}:=e_{j}^{i}-e_{j}^{j}, \quad i \neq j$, with the Frobenius structure determined by the coboundary of the 1-cochain $\lambda_{n}=-\sum_{i, j: i \neq j} \mu_{i} z_{j}^{i}$, where $\left\{z_{j}^{i}\right\}, i \neq j$, is the basis in $\mathfrak{g}_{0}^{*}(n)$, dual to the basis $\left\{Z_{j}^{i}\right\}$ in $\mathfrak{g}_{0}(n), z_{j}^{i}\left(Z_{l}^{k}\right)=\delta_{l}^{i} \delta_{j}^{k}$.

Proof. An artificial introduction of a small parameter $c$ by a rescaling $\mu_{i} \mapsto c^{-1} \mu_{i}$ in the formula for the $R$-matrix $\hat{R}$ in Proposition 2.2 gives

$$
R=\mathbb{1} \otimes \mathbb{1}+c r,
$$

where $r$ is given by (4.19). 
The $n(n-1)$ matrices $Z_{j}^{i}:=e_{j}^{i}-e_{j}^{j}, i \neq j$, form an associative subalgebra of the matrix algebra,

$$
Z_{i}^{j} Z_{l}^{k}=\left(\delta_{l}^{j}-\delta_{l}^{i}\right)\left(Z_{i}^{k}-Z_{i}^{l}\right)
$$

(we set $Z_{i}^{i}=0$ for all $i$ ); with respect to the commutators these matrices form a Lie subalgebra $\mathfrak{g}_{0}(n)$ of the Lie algebra $\mathfrak{g l}(n), \mathfrak{g}_{0}(n) \subset \mathfrak{g l}(n)$ :

$$
\begin{aligned}
& {\left[Z_{j}^{i}, Z_{i}^{j}\right]=Z_{i}^{j}-Z_{j}^{i}, \quad\left[Z_{i}^{j}, Z_{i}^{k}\right]=Z_{i}^{j}-Z_{i}^{k},} \\
& {\left[Z_{j}^{i}, Z_{k}^{j}\right]=Z_{k}^{j}-Z_{k}^{i}, \quad i \neq j \neq k \neq i,}
\end{aligned}
$$

all other brackets vanish. The skew-symmetric solution (4.19) of the cYB equation,

$$
r=\sum_{i, j: i<j} \frac{Z_{j}^{i} \wedge Z_{i}^{j}}{\mu_{i}-\mu_{j}}
$$

is non-degenerate on the carrier subalgebra $\mathfrak{g}_{0}(n)$. The carrier subalgebra $\mathfrak{g}_{0}(n)$ is necessarily quasi-Frobenius, having a 2-cocycle $\omega$ given by the inverse of the $r$-matrix, that is,

$$
\omega\left(Z_{A}, Z_{B}\right)=r_{A B}, \quad \text { where } \quad r^{A B} r_{B C}=\delta_{C}^{A}, \quad r=\sum_{A, B} r^{A B} Z_{A} \wedge Z_{B}
$$

We have

$$
\omega\left(Z_{j}^{i}, Z_{l}^{k}\right)=-\left(\mu_{i}-\mu_{j}\right) \delta_{i}^{l} \delta_{k}^{j} .
$$

It is easy to check that the 2-cycle $\omega$ is a coboundary,

$$
\omega\left(Z_{j}^{i}, Z_{l}^{k}\right)=\lambda_{n}\left(\left[Z_{j}^{i}, Z_{l}^{k}\right]\right), \quad \lambda_{n}=-\sum_{i, j: i \neq j} \mu_{i} z_{j}^{i} \in \mathfrak{g}_{0}^{*}(n),
$$

thus the subalgebra $\mathfrak{g}_{0}(n)$ is Frobenius.

The "Frobenius" $r$-matrix (4.19) (and its quantization) was considered in the work [2].

Proposition 4.3. The skew-symmetric rime classical r-matrix (4.19), $r=\sum_{i<j}\left(\mu_{i}-\mu_{j}\right)^{-1} Z_{j}^{i} \wedge Z_{i}^{j}$, where $\mu=\left(\mu_{1}, \mu_{2}, \ldots, \mu_{n}\right)$ is an arbitrary 
vector such that $\mu_{i} \neq \mu_{j}$ belongs to the orbit of the parameter-free classical r-matrix

$$
b=\sum_{i, j: i<j} \sum_{k=1}^{j-i} e_{i}^{i+k} \wedge e_{j}^{j-k+1} .
$$

More precisely,

$$
r=\operatorname{Ad}_{X_{\mu}} \otimes \operatorname{Ad}_{X_{\mu}}(b)
$$

where the element $X_{\mu} \in \mathrm{GL}(n)$ is defined by $\left(X_{\mu}\right)_{k}^{j}=e_{k-1}\left(\mu_{1}, \ldots\right.$, $\left.\hat{\mu}_{j}, \ldots, \mu_{n}\right){ }^{3}$

Proof. The equality $r=\operatorname{Ad}_{X_{\mu}} \otimes \operatorname{Ad}_{X_{\mu}}(b)$ is equivalent to a set of relations for the elementary symmetric functions $e_{i}$,

$$
\left(X_{\mu} \otimes X_{\mu}\right) b=r\left(X_{\mu} \otimes X_{\mu}\right) \quad \Leftrightarrow \quad \sum_{r, s} e_{r-1}^{\hat{i}} e_{s-1}^{\hat{j}} b_{k l}^{r s}=\sum_{a, b} r_{a b}^{i j} e_{k-1}^{\hat{a}} e_{l-1}^{\hat{b}},
$$

where

$$
\begin{aligned}
b_{a b}^{i j} & =\sum_{k=1}^{j-i} \delta_{b}^{j-k+1} \delta_{a}^{i+k}-\sum_{k=1}^{i-j} \delta_{a}^{i-k+1} \delta_{b}^{j+k} \text { and } \\
r_{a b}^{i j} & =\left\{\begin{array}{cc}
\left(\delta_{a}^{i} \delta_{b}^{i}+\delta_{a}^{j} \delta_{b}^{j}-\delta_{a}^{i} \delta_{b}^{j}-\delta_{a}^{j} \delta_{b}^{i}\right) /\left(\mu_{i}-\mu_{j}\right), & i \neq j, \\
0, & i=j .
\end{array}\right.
\end{aligned}
$$

Both operators $b_{a b}^{i j}$ and $r_{a b}^{i j}$ are symmetric in the lower indices and antisymmetric in the upper indices, that is,

$$
P b=-b, \quad b P=b \text { and } P r=-r, \quad r P=r .
$$

Equations (4.29) have the following form

$$
-\sum_{s \geq 1}\left(e_{b+s-2}^{\hat{i}} e_{a-s-1}^{\hat{j}}-e_{b+s-2}^{\hat{j}} e_{a-s-1}^{\hat{i}}\right)=\frac{1}{\mu_{i}-\mu_{j}}\left(e_{a-1}^{\hat{i}}-e_{a-1}^{\hat{j}}\right)\left(e_{b-1}^{\hat{i}}-e_{b-1}^{\hat{j}}\right) .
$$

\footnotetext{
${ }^{3}$ This matrix is the same $X$ as in Proposition 3.1 but depending on variables $\mu_{i}$.
} 
Due to (3.11), the left-hand side of (4.31) equals

$$
-\frac{1}{\mu_{i}-\mu_{j}}\left(\mu_{i} e_{a-2}^{\hat{i}}-\mu_{j} e_{a-2}^{\hat{j}}\right)\left(e_{b-1}^{\hat{i}}-e_{b-1}^{\hat{j}}\right) .
$$

The right-hand side of (4.31) equals (4.32) as well because $e_{a-1}^{\hat{i}}=e_{a-1}-$ $\mu_{i} e_{a-2}^{\hat{i}}$.

As in the non-skew-symmetric case, in the infinite dimension the operators $b$ and $r$ are in general not equivalent.

The $\mathfrak{s l}(\boldsymbol{n}) \mathbf{c Y B}$ solution. Let $I=\sum_{i=1}^{n} e_{i}^{i}$ be the central element of $\mathfrak{g l}(n)$. The generators $\tilde{Z}_{j}^{i}=Z_{j}^{i}+\frac{1}{n} I \in \mathfrak{s l}(n)$ satisfy the same relations (4.22) as $Z_{j}^{i}$ thus they form a subalgebra $\tilde{\mathfrak{g}}_{0}(n)$ of the Lie algebra $\mathfrak{s l}(n)$ which is isomorphic to $\mathfrak{g}_{0}(n), \tilde{\mathfrak{g}}_{0}(n) \simeq \mathfrak{g}_{0}(n)$. This isomorphism gives rise to another solution $\tilde{r} \in \mathfrak{s l}(n) \wedge \mathfrak{s l}(n)$ of the cYB equation,

$$
\tilde{r}=\sum_{i, j: i<j} \frac{\tilde{Z}_{j}^{i} \wedge \tilde{Z}_{i}^{j}}{\mu_{i}-\mu_{j}} \in \mathfrak{s l}(n) \wedge \mathfrak{s l}(n) .
$$

We have the following lemma about the carrier Lie algebra of $\tilde{r}$ (the Lie subalgebra of $\mathfrak{s l}(n)$ spanned by the generators $\left.\tilde{Z}_{j}^{i}\right)$.

Lemma 4.2. The subalgebra $\tilde{\mathfrak{g}}_{0}(n) \subset \mathfrak{s l}(n)$ of dimension $\operatorname{dim} \tilde{\mathfrak{g}}_{0}(n)=n(n-$ 1) is isomorphic to the maximal parabolic subalgebra $\mathfrak{p}$ of $\mathfrak{s l}(n)$ obtained by deleting the first negative root.

Proof. The vector $v=\sum_{i=1}^{n} e_{i}$ is an eigenvector for all elements $\tilde{Z}_{j}^{i}$,

$$
\tilde{Z}_{j}^{i}(v)=\frac{1}{n} v \quad \text { for all } i \text { and } j, \quad i \neq j .
$$

In a basis in which the first vector is $v$, the linear span of the generators $\tilde{Z}_{j}^{i}$ is

$$
\left(\begin{array}{cccc}
* & * & \ldots & * \\
0 & * & & * \\
\vdots & \vdots & & \vdots \\
0 & * & \ldots & *
\end{array}\right)
$$

with the traceless condition. The comparison of dimensions finishes the proof. 
Gerstenhaber and Giaquinto [14] found a classical $r$-matrix $b_{\mathrm{CG}}$ which they called "boundary" because it lies in the closure of the solution space of the YB equation. The cYB solution $b_{\mathrm{CG}}$ corresponds to a Frobenius subalgebra $(\mathfrak{p}, \Omega)$, where $\mathfrak{p}$ is the parabolic subalgebra of $\mathfrak{s l}(n)$ as above and the 2-cocycle $\Omega$ is a coboundary,

$$
\Omega=\delta \lambda_{b_{\mathrm{CG}}}, \quad \lambda_{b_{\mathrm{CG}}}=\sum_{i=1}^{n}\left(e_{i+1}^{i}\right)^{*} \in \mathfrak{p}^{*} .
$$

The $r$-matrix $b_{\mathrm{CG}}$ is a twist of $b$ (see [8]).

Since the carriers of $\tilde{r}$ and $b_{\mathrm{CG}}$ are isomorphic, the $r$-matrices are equivalent. We shall now prove that the same matrix $X_{\mu}$ transforms $b_{\mathrm{CG}}$ into $\tilde{r}$.

Proposition 4.4. The boundary classical $r$-matrix $b_{\mathrm{CG}} \in \mathfrak{s l}(n) \wedge \mathfrak{s l}(n)$,

$$
b_{\mathrm{CG}}=\sum_{i, j}\left(1-\frac{j}{n}\right) e_{i}^{i} \wedge e_{j}^{j+1}+\sum_{i, j: i<j} \sum_{k=1}^{j-i} e_{i}^{i+k} \wedge e_{j}^{j-k+1},
$$

transforms into the $c Y B$ solution $\tilde{r} \in \mathfrak{s l}(n) \wedge \mathfrak{s l}(n)$,

$$
\tilde{r}=\sum_{i, j: i<j} \frac{\tilde{Z}_{j}^{i} \wedge \tilde{Z}_{i}^{j}}{\mu_{i}-\mu_{j}}, \text { where } \tilde{Z}_{j}^{i}=e_{j}^{i}-e_{j}^{j}+\frac{1}{n} \sum_{i=1}^{n} e_{i}^{i},
$$

by a change of basis with the matrix $X_{\mu} \in \mathrm{GL}(n)$,

$$
\tilde{r}=\operatorname{Ad}_{X_{\mu}} \otimes \operatorname{Ad}_{X_{\mu}}\left(b_{\mathrm{CG}}\right) .
$$

Proof. Due to Proposition 4.3 we have $r=A \mathrm{~d}_{X_{\mu}} \otimes A \mathrm{~d}_{X_{\mu}}(b)$. The cYB solution $b_{\mathrm{CG}}$ is the sum of $b$ and other terms, $b_{\mathrm{CG}}=b+\sum_{i, j}\left(1-\frac{j}{n}\right) e_{i}^{i} \wedge$ $e_{j}^{j+1}$. Therefore it is enough to show that $\tilde{r}-r=\operatorname{Ad}_{X_{\mu}} \otimes \operatorname{Ad}_{X_{\mu}}\left(b_{\mathrm{CG}}-b\right)$. One has

$$
\tilde{r}-r=\frac{1}{n} I \wedge \sum_{i, j: i \neq j} \frac{Z_{i}^{j}}{\mu_{i}-\mu_{j}}, \quad b_{\mathrm{CG}}-b=I \wedge \sum_{j}\left(1-\frac{j}{n}\right) e_{j}^{j+1} .
$$

Thus we have to show that

$$
X_{\mu} \sum_{j}\left(1-\frac{j}{n}\right) e_{j}^{j+1}=\frac{1}{n} \sum_{i, j: i \neq j} \frac{Z_{i}^{j}}{\mu_{i}-\mu_{j}} X_{\mu}
$$


which amounts to the following identities for the elementary symmetric functions:

$$
\left(1-\frac{b-1}{n}\right) e_{b-2}^{\hat{i}}=\frac{1}{n} \sum_{j: j \neq i} \frac{e_{b-1}^{\hat{j}}-e_{b-1}^{\hat{i}}}{\mu_{i}-\mu_{j}} .
$$

Replacing, in the right-hand side, $e_{b-1}^{\hat{j}}$ by $e_{b-1}^{\hat{i} \hat{j}}+\mu_{i} e_{b-2}^{\hat{i} \hat{j}}, e_{b-1}^{\hat{i}}$ by $e_{b-1}^{\hat{i} \hat{j}}+$ $\mu_{j} e_{b-2}^{\hat{i} \hat{j}}$ and noticing that $\sum_{i} e_{c}^{\hat{i}}=(n-c) e_{c}, c=1,2, \ldots, n$ (for the elementary symmetric functions in $n$ variables) finishes the proof.

The passage to the $\mathfrak{s l}(n)$ solution is another instance of the representation change. The general representation change (4.6) produces the following effect on the numerical $r$-matrix (4.27):

$$
b \mapsto b-c \eta^{(0)} \wedge \mathbb{1}
$$

where $\eta^{(0)}$ is the generator of the invariance group of the $r$-matrix (4.27),

$$
\eta^{(0)}=\sum(n-j) e_{j}^{j+1}
$$

The representation change and the operation (4.10) produce the same 1-parametric family (4.43) of skew-symmetric $r$-matrices. The choice $c=$ $-1 / n$ corresponds to the $r$-matrix $b_{\mathrm{CG}}$.

\section{Bézout operators}

The Bézout operator [5] is the following endomorphism $\mathfrak{b}^{(0)}$ of the space $\mathfrak{P}$ of polynomials of two variables $x$ and $y$ :

$$
\mathfrak{b}^{(0)} f(x, y)=\frac{f(x, y)-f(y, x)}{x-y} \quad \text { or } \quad \mathfrak{b}^{(0)}=\frac{1}{x-y}(I-P),
$$

where $I$ is the identity operator and $P$ is a permutation, $\operatorname{Pf}(x, y)=f(y, x)$. For any natural $n$, the subspace $\mathfrak{P}_{n}$ of polynomials of degree less than $n$ in $x$ and less than $n$ in $y$ is invariant with respect to the operator $\mathfrak{b}^{(0)}$. The matrix of the restriction of $\mathfrak{b}^{(0)}$ onto $\mathfrak{P}_{n}$, written in the basis $\left\{x^{a} y^{b}\right\}$ of powers (in the decreasing order) coincides with the operator (4.27). 
The non-skew-symmetric matrix (4.3) is the matrix of the operator

$$
\mathfrak{b}=\frac{x}{x-y}(I-P)
$$

in this basis. The rime bases are formed by the non-normalized Lagrange polynomials $\left\{l_{i}(x) l_{j}(y)\right\}, l_{i}(t)=\prod_{s: s \neq i}\left(t-\phi_{s}\right)$, at points $\left\{\phi_{i}\right\}, i=1$, $2, \ldots, n$.

We shall call the operators $\mathfrak{b}^{(0)}$ and $\mathfrak{b}$ Bézout $r$-matrices. The Bézout $r$-matrices were rediscovered in several different contexts related to the Yang-Baxter equation (except the fact that they are the Cremmer-Gervais $r$-matrices, they appear, for instance, in [7] and [19]).

The standard $r$-matrix $r^{(s)}$, for the choice of the multi-parameters for which it can be non-trivially rimed (see the Remark at the end of Section 3), has the following form in terms of polynomials

$$
r^{(s)}: x^{i} y^{j} \mapsto \theta(i-j) x^{i} y^{j}-\theta(j-i) x^{j} y^{i} .
$$

The subspaces $\mathfrak{P}_{n}$ are invariant with respect to $r^{(s)}$.

The properties of the Bézout $r$-matrices $\mathfrak{b}^{(0)}$ and $\mathfrak{b}$ (and of the operator $r^{(s)}$ ) become more transparent when they are viewed as operators on polynomials. In particular,

$$
\begin{gathered}
\left(\mathfrak{b}^{(0)}\right)^{2}=0, \quad \mathfrak{b}^{(0)} P=-\mathfrak{b}^{(0)}, \quad P \mathfrak{b}^{(0)}=\mathfrak{b}^{(0)}, \quad \mathfrak{b}^{(0)}+\mathfrak{b}_{21}^{(0)}=0, \\
\mathfrak{b}^{2}=\mathfrak{b}, \quad \mathfrak{b} P=-\mathfrak{b}, \quad \mathfrak{b}+\mathfrak{b}_{21}=I-P \\
\left(r^{(s)}\right)^{2}=r^{(s)}, \quad r^{(s)} P=-r^{(s)}, \quad r^{(s)}+r_{21}^{(s)}=I-P .
\end{gathered}
$$

The description of the invariance groups of the operators $\mathfrak{b}^{(0)}$ and $\mathfrak{b}$ is especially transparent when these operators are viewed as operators on the spaces of polynomials. Let $\partial_{x}$ and $\partial_{y}$ be the derivatives in $x$ and $y$. We have $\left(\partial_{x}+\partial_{y}\right)\left(\frac{1}{x-y}\right)=0$ which implies that $\partial_{x}$ is the generator of the invariance group of $\mathfrak{b}^{(0)}$; the group is formed by translations. Similarly, $\left(x \partial_{x}+y \partial_{y}\right)\left(\frac{x}{x-y}\right)=0$ which implies that $x \partial_{x}$ is the generator of the invariance group of $\mathfrak{b}$; the group is formed by dilatations. The operation (4.10) implies that the operators

$$
\mathfrak{b}^{(0)}+c\left(\partial_{x}-\partial_{y}\right), \quad \mathfrak{b}+c\left(x \partial_{x}-y \partial_{y}\right)
$$

are solutions of the cYBe (the quantum version is easy as well) for an arbitrary constant $c$. 


\subsection{Non-homogeneous associative classical Yang-Baxter equation}

The operators $\mathfrak{b}^{(0)}, \mathfrak{b}$ and $r^{(s)}$ satisfy an equation stronger than the cYBe. For an endomorphism $r$ of $V \otimes V$, define

$$
r \circ r:=r_{12} r_{13}+r_{13} r_{23}-r_{23} r_{12}, \quad r \circ^{\prime} r:=r_{13} r_{12}+r_{23} r_{13}-r_{12} r_{23} .
$$

The equation $r \circ r=0$ (as well as $r \circ^{\prime} r=0$ ) is called associative classical Yang-Baxter equation (acYBe) $[1,20]$.

We introduce a non-homogeneous associative classical Yang-Baxter equation (nhacYBe):

$$
r \circ r=c r_{13},
$$

where $c$ is a constant.

Let $\mathcal{F}$ be the space of polynomials in one variable. For the space $\mathcal{F} \otimes \mathcal{F}$ of polynomials in two variables, we denote by $x$ (respectively, $y$ ) the generator of the first (respectively, second) copy of $\mathcal{F}$. For $\mathcal{F} \otimes \mathcal{F} \otimes \mathcal{F}$, the generators are denoted by $x, y$ and $z$.

Lemma 5.1. 1. Let $M$ be an operator on the space $\mathcal{F} \otimes \mathcal{F}$. Assume that

$$
\begin{gathered}
M(x f)=f+y M(f), \\
M(y f)=-f+x M(f)
\end{gathered}
$$

for an arbitrary $f \in \mathcal{F} \otimes \mathcal{F}$. Then ${ }^{4}$

$$
\begin{aligned}
& M \circ M(x F)=z M \circ M(F), \quad M \circ M(y F)=x M \circ M(F), \\
& M \circ M(z F)=y M \circ M(F)
\end{aligned}
$$

for an arbitrary $F \in \mathcal{F} \otimes \mathcal{F} \otimes \mathcal{F}$.

2. The operator $M=\mathfrak{b}^{(0)}$ verifies (5.10) and (5.11).

3. Moreover, the unique solution of equations (5.10) and (5.11) (for the operator $M$ on the space $\mathcal{F} \otimes \mathcal{F})$ together with the "initial" condition $M(1)=0$ is $M=\mathfrak{b}^{(0)}$.

Proof. A direct calculation.

\footnotetext{
${ }^{4}$ Equation $M \circ M(x F)=z M \circ M(F)$ follows from (5.10) alone.
} 
Proposition 5.1. 1. The Bézout operator $\mathfrak{b}^{(0)}$ satisfies the acYBe.

2. The Bézout operator $\mathfrak{b}$ and the operator $r^{(s)}$ satisfy the nhacYBe with $c=1$.

Proof. A direct calculation for $\mathfrak{b}^{(0)}$. Another way is to notice that the relations (5.12) for $M=\mathfrak{b}^{(0)}$ reduce the verification of $\mathfrak{b}^{(0)} \circ \mathfrak{b}^{(0)}(F)=0$ for a monomial $F \in \mathcal{F} \otimes \mathcal{F} \otimes \mathcal{F}$ to the case $F=1$, which is trivial.

For the Bézout operator $\mathfrak{b} \equiv x \mathfrak{b}^{(0)}(x$ here is the operator of multiplication by $x$ ), we have, for an arbitrary $F \in \mathcal{F} \otimes \mathcal{F} \otimes \mathcal{F}$,

$$
\begin{aligned}
\mathfrak{b} \circ \mathfrak{b}(F) & =x \mathfrak{b}_{12}^{(0)}\left(x \mathfrak{b}_{13}^{(0)}(F)\right)+x \mathfrak{b}_{13}^{(0)}\left(y \mathfrak{b}_{23}^{(0)}(F)\right)-y \mathfrak{b}_{23}^{(0)}\left(x \mathfrak{b}_{12}^{(0)}(F)\right) \\
& =x\left(\mathfrak{b}_{13}^{(0)}(F)+y \mathfrak{b}_{12}^{(0)} \mathfrak{b}_{13}^{(0)}(F)\right)+x y \mathfrak{b}_{13}^{(0)} \mathfrak{b}_{23}^{(0)}(F)-x y \mathfrak{b}_{23}^{(0)} \mathfrak{b}_{12}^{(0)}(F) \\
& =x \mathfrak{b}_{13}^{(0)}(F)+x y \mathfrak{b}^{(0)} \circ \mathfrak{b}^{(0)}(F)=\mathfrak{b}_{13}(F) .
\end{aligned}
$$

We used equation (5.10) for $\mathfrak{b}^{(0)}$ in the second equality.

For the operator $r^{(s)}$, the identity

$$
\theta(i-k) \theta(i-j)+\theta(i-k) \theta(j-k)-\theta(i-j) \theta(j-k)=\theta(i-k)
$$

for the step function $\theta$ is helpful.

In each of cases (5.4-5.6), the operator $r$ satisfies a quadratic equation $r^{2}=u_{1} r+u_{2} I$, the relation $r+r_{21}=\alpha P+\beta I$ with some constants $\alpha$ and $\beta$ and the nhacYBe with some constant $c$. Several general comments about relations between the constants appearing in these equations are in order here.

1. Assume that an $r$-matrix (a solution of the cYBe) satisfies $r \circ r=c r_{13}$. Then $r \circ^{\prime} r=c r_{13}$. Taking the combinations $\left(r \circ r-c r_{13}\right)-P_{23}\left(r \circ^{\prime}\right.$ $\left.r-c r_{13}\right) P_{23}$ and $\left(r \circ r-c r_{13}\right)-P_{12}\left(r \circ r-c r_{13}\right) P_{12}$, we find

$$
r_{13}(S r)_{23}-(S r)_{23} r_{12}=c\left(r_{13}-r_{12}\right), \quad(S r)_{12} r_{13}-r_{23}(S r)_{12}=c\left(r_{13}-r_{23}\right),
$$

where $(S r)_{12}:=r_{12}+r_{21}$. If $(S r)_{12}=\alpha P_{12}+\beta I$ with some constants $\alpha$ and $\beta$, as in (5.4)-(5.6), then it follows from (5.15) that $(\beta-c)$ 
$\left(r_{13}-r_{12}\right)=0$ thus

$$
c=\beta \text {. }
$$

This explains the value of the constant $c$ in Lemma 5.1.

2. For an endomorphism $r$ of $V \otimes V$, assume that $r \circ r=\beta r_{13}$ and $(S r)_{12}=\alpha P_{12}+\beta I$. Then

$$
\begin{aligned}
& P_{23}\left(r \circ r-\beta r_{13}\right) P_{23}=r_{13} r_{12}+r_{12} r_{32}-r_{32} r_{13}-\beta r_{12} \\
& \quad=r_{13} r_{12}+r_{12}\left(\alpha P_{23}+\beta I-r_{23}\right)-\left(\alpha P_{23}+\beta I-r_{23}\right) r_{13} \\
& \quad-\beta r_{12}=r \circ^{\prime} r-\beta r_{13} .
\end{aligned}
$$

Thus, if $(S r)_{12}=\alpha P_{12}+\beta I$ then $r \circ r=\beta r_{13}$ implies $r \circ^{\prime} r=\beta r_{13}$.

3. Assume that $r \circ r=c r_{13}$ for an endomorphism $r$ of $V \otimes V$. Then for $\tilde{r}=r+a I+b P, a$ and $b$ are constants, we have

$$
\tilde{r} \circ \tilde{r}=(c+2 a) \tilde{r}_{13}+b P_{13}(S r)_{23}-a(a+c) I-b c P_{13}+b^{2} P_{23} P_{12} .
$$

If, in addition, $(S r)_{12}=\alpha P_{12}+\beta I$, then

$$
\tilde{r} \circ \tilde{r}=(c+2 a) \tilde{r}_{13}-a(c+a) I+b(\beta-c) P_{13}+b(\alpha+b) P_{23} P_{12} .
$$

This shows that the equation $r \circ r=c_{1} r_{13}+c_{2} I+c_{3} P_{13}+c_{4} P_{23} P_{12}$, $c_{1}, c_{2}, c_{3}$ and $c_{4}$ are constants, reduces to $r \circ r=\tilde{c}_{1} r_{13}+\tilde{c}_{3} P_{13}$ by a shift $r \mapsto r+a I+b P$.

If $r \circ r=\beta r_{13}$ and $(S r)_{12}=\alpha P_{12}+\beta I$ then

$$
\tilde{r} \circ \tilde{r}=(\beta+2 a) \tilde{r}_{13}-a(\beta+a) I+b(\alpha+b) P_{23} P_{12} .
$$

The combination $P_{23} P_{12}$ does not appear for $b=0$ or $b=-\alpha$. The choice $b=-\alpha$ corresponds, modulo a shift of $r$ by a multiple of $I$, to $r \mapsto r_{21}$, so we consider only $b=0$. Then, with the choice $a=-\beta$ we find that the operator $\tilde{r}=r-\beta I$ satisfies the nhacYBe (and $(S r)_{12}=$ $\left.\alpha P_{12}-\beta I\right)$. For the choice $a=-\beta / 2$ we find that the operator $\tilde{r}=$ $r-\frac{\beta}{2} I$ satisfies

$$
\tilde{r} \circ \tilde{r}=\frac{\beta^{2}}{4}, \quad(S \tilde{r})_{12}=\alpha P_{12}
$$


In particular, the operator

$$
\tilde{\mathfrak{b}}=\frac{x+y}{2(x-y)} I-\frac{x}{x-y} P
$$

satisfies (5.21) with $\beta=1$ and $\alpha=-1$. Also, $\tilde{\mathfrak{b}}^{2}=\frac{1}{4} I$.

4. Assume that $r^{2}=u r+v$ and $r_{12}+r_{21}=\alpha P_{12}+\beta I$ for an endomorphism $r$ of $V \otimes V$. Squaring the relation $r_{12}-\beta I=\alpha P_{12}-r_{21}$ and using the same relation again, we obtain

$$
(u-\beta)\left(2 r_{12}-\beta I-\alpha P_{12}\right)=0 .
$$

Thus, if $r$ is not a linear combination of $I$ and $P$ then

$$
u=\beta \text {. }
$$

5. Assume that $r \circ r=c r_{13}$ and $r P=-r$ for an endomorphism $r$ of $V \otimes$ $V$. The nhacYBe has the following equivalent form:

$$
\left[r_{13}, r_{23}\right]=\left(r_{12}-c I\right) r_{13} P_{23}
$$

Indeed,

$$
r_{13} r_{23}-r_{23} r_{13}=\left(-r_{13} r_{23}+r_{23} r_{12}\right) P_{23}=\left(r_{12}-c I\right) r_{13} P_{23}
$$

Here in the first equality we used $r_{23} P_{23}=-r_{23}$ and moved $P_{23}$ to the right; in the second equality we used the nhacYBe $r \circ r=c r_{13}$.

\subsection{Linear quantization}

Consider an algebra with three generators $r_{12}, r_{13}$ and $r_{23}$ and relations

$$
\begin{aligned}
r_{13} r_{23} & =r_{23} r_{12}-r_{12} r_{13}+\beta r_{13}, \\
r_{13} r_{12} & =r_{12} r_{23}-r_{23} r_{13}+\beta r_{13}, \\
r_{12}^{2} & =\beta r_{12}+v, r_{13}^{2}=\beta r_{13}+v, r_{23}^{2}=\beta r_{23}+v
\end{aligned}
$$

Choose an order, say, $r_{13}>r_{23}>r_{12}$. Consider (5.27) as ordering relations. The overlaps in (5.27) lead to exactly one more relation:

$$
r_{23} r_{12} r_{23}=r_{12} r_{23} r_{12}
$$


Thus the algebra in question is 12-dimensional (it follows from (5.27) and (5.28) that a general element of the algebra is a product $A B$ of an element $A$ of the Hecke algebra generated by $r_{12}$ and $r_{23}$ and a polynomial $B$, of degree less than 2 , in $r_{13}$ ).

We conclude that the nhacYBe together with the quadratic equation for $r$ imply the YBe. Note that the other form of the YBe also follows:

$$
\begin{aligned}
r_{23} r_{13} r_{12}-r_{12} r_{13} r_{23}= & \left(r_{12} r_{23}-r_{13} r_{12}+\beta r_{13}\right) r_{12} \\
& -r_{12}\left(r_{23} r_{12}-r_{12} r_{13}+\beta r_{13}\right) r_{23} \\
= & -r_{13}\left(\beta r_{12}+v\right)+\beta r_{13} r_{12}+\left(\beta r_{12}+v\right) r_{13} \\
& -\beta r_{12} r_{13}=0 .
\end{aligned}
$$

Here in the first equality both nhacYBe for $r$ were used; the quadratic relation for $r$ was used in the second equality.

Therefore, the quantization of such $r$-matrix is "linear" ${ }^{5}$ : a combination

$$
R=I+\lambda r
$$

where $\lambda$ is an arbitrary constant, satisfies the $\mathrm{YBe} R_{12} R_{13} R_{23}=R_{23} R_{13} R_{12}$.

\subsection{Algebraic meaning}

We shall clarify the algebraic meaning of the non-homogeneous associative classical Yang-Baxter equation in the general context of associative algebras.

Let $\mathfrak{A}$ be an algebra. Let $r \in \mathfrak{A} \otimes \mathfrak{A}$. The operation

$$
\delta^{(0)}: \mathfrak{A} \longrightarrow \mathfrak{A} \otimes \mathfrak{A}, \quad \delta^{(0)}(u)=(u \otimes 1) r-r(1 \otimes u)
$$

(the algebra $\mathfrak{A}$ does not need to be unital, $(u \otimes 1)(a \otimes b)$ stands for $u a \otimes b$ and $(a \otimes b)(u \otimes 1)$ for $a u \otimes b)$ is coassociative if and only if [1]

$$
(u \otimes 1 \otimes 1)\left(r \circ^{\prime} r\right)=\left(r \circ^{\prime} r\right)(1 \otimes 1 \otimes u) \quad \forall u \in \mathfrak{A} .
$$

In particular, $\delta^{(0)}$ is coassociative if $\left(r \circ^{\prime} r\right)=0$.

\footnotetext{
${ }^{5}$ It was noted in $[8]$ that the operator $\mathfrak{b}^{(0)}$ satisfies both forms of the YBe, squares to zero and that its quantization has the simple form (5.30).
} 
Assume now that the algebra $\mathfrak{A}$ is unital. Define the operations $\delta$ and $\tilde{\delta}: \mathfrak{A} \rightarrow \mathfrak{A} \otimes \mathfrak{A}$,

$$
\begin{gathered}
\delta(u):=(u \otimes 1) r-r(1 \otimes u)-c(u \otimes 1), \\
\tilde{\delta}(u):=(u \otimes 1) r-r(1 \otimes u)+c(1 \otimes u),
\end{gathered}
$$

where $c$ is a constant.

Proposition 5.2. The coassociativity of each of the operations $\delta$ and $\tilde{\delta}$ is equivalent to

$$
(u \otimes 1 \otimes 1)\left(r \circ^{\prime} r-c r_{13}\right)=\left(r \circ^{\prime} r-c r_{13}\right)(1 \otimes 1 \otimes u) \quad \forall u \in \mathfrak{A} .
$$

Proof. A straightforward calculation.

In particular, the operations $\delta$ and $\tilde{\delta}$ are coassociative if $r \circ^{\prime} r=c r_{13}$.

The map (5.31) has the following property:

$$
\delta^{(0)}(u v)=(u \otimes 1) \delta^{(0)}(v)+\delta^{(0)}(u)(1 \otimes v) ;
$$

that is, $\delta^{(0)}$ is a derivation with respect to the standard structure of $\mathfrak{A} \otimes \mathfrak{A}$ as a bi-module over $\mathfrak{A}, u U:=(u \otimes 1) U$ and $U u:=U(1 \otimes u)$ for $u \in \mathfrak{A}$ and $U \in \mathfrak{A} \otimes \mathfrak{A}$.

For the operations $\delta$ and $\tilde{\delta}$, the analogue of the property (5.36) reads

$$
\begin{aligned}
& \delta(u v)=(u \otimes 1) \delta(v)+\delta(u)(1 \otimes v)+c(u \otimes v) \\
& \tilde{\delta}(u v)=(u \otimes 1) \tilde{\delta}(v)+\tilde{\delta}(u)(1 \otimes v)-c(u \otimes v)
\end{aligned}
$$

\subsection{Rota-Baxter operators}

Let $\mathcal{A}$ be an algebra. An operator $\mathfrak{r}: \mathcal{A} \rightarrow \mathcal{A}$ is called Rota-Baxter operator of weight $\alpha$ if

$$
\mathfrak{r}(A) \mathfrak{r}(B)+\alpha \mathfrak{r}(A B)=\mathfrak{r}(\mathfrak{r}(A) B+A \mathfrak{r}(B))
$$

for arbitrary $A, B \in \mathcal{A}$ ( $\alpha$ is a constant). We refer to [22] for further information about the Rota-Baxter operators.

The Rota-Baxter operators of weight zero are closely related to the acYBe [23]. It turns out that the Rota-Baxter operators of non-zero weight are 
related to the nhacYBe. We shall discuss this relation and calculate the Rota-Baxter operators corresponding to the Bézout operators.

It is surprising that the Bézout operators, which rather have the sense of derivatives, become, being interpreted as operators on matrix algebras, the Rota-Baxter operators which are designed to axiomatize the properties of indefinite integrations and summations.

1. For an endomorphism $r$ of $V \otimes V$, define two endomorphisms, $\mathfrak{r}$ and $\mathfrak{r}^{\prime}$, of the matrix algebra $\operatorname{Mat}(V)$ :

$$
\mathfrak{r}(A)_{1}:=\operatorname{Tr}_{2}\left(r_{12} A_{2}\right), \quad \mathfrak{r}^{\prime}(A)_{2}:=\operatorname{Tr}_{1}\left(r_{12} A_{1}\right), \quad A \in \operatorname{Mat}(V),
$$

where $\operatorname{Tr}_{i}$ is the trace in the copy number $i$ of the space $V$.

Assume that $r$ satisfies the nhacYBe (5.9). Multiplying (5.9) by $A_{2} B_{3}$, $A, B \in \operatorname{Mat}(V)$, and taking traces in the spaces 2 and 3 , we find

$$
\mathfrak{r}(A) \mathfrak{r}(B)+\mathfrak{r}\left(\mathfrak{r}^{\prime}(A) B\right)-\mathfrak{r}(A \mathfrak{r}(B))=c \operatorname{Tr}(A) \mathfrak{r}(B) .
$$

Assume, in addition, that $r_{12}+r_{21}=\alpha P_{12}+\beta I$. Then

$$
\mathfrak{r}(A)+\mathfrak{r}^{\prime}(A)=\alpha A+\beta \operatorname{Tr}(A) \mathbb{1} .
$$

If $c=\beta$ then expressing $\mathfrak{r}^{\prime}(A)$ by (5.42) and substituting into (5.41), we find that the term with $\operatorname{Tr}(A)$ drops out and $\mathfrak{r}$ is the Rota-Baxter operator of weight $\alpha$ on the algebra of matrices. Similarly, $\mathfrak{r}^{\prime}$ is the Rota-Baxter operator of weight $\alpha$ as well.

2. We shall calculate the Rota-Baxter operators corresponding to the Bézout operators in the polynomial basis.

The action of the operator $\mathfrak{b}^{(0)}$ on monomials $x^{k} y^{l}$ reads

$$
\mathfrak{b}^{(0)}\left(x^{k} y^{l}\right)= \begin{cases}-\left(x^{l-1} y^{k}+x^{l-2} y^{k+1}+\cdots+x^{k} y^{l-1}\right), & k<l, \\ 0, & k=l, \\ x^{k-1} y^{l}+x^{k-2} y^{l+1}+\cdots+x^{l} y^{k-1}, & k>l .\end{cases}
$$

The action of the operator $\mathfrak{b}$ on monomials $x^{k} y^{l}$ reads

$$
\mathfrak{b}\left(x^{k} y^{l}\right)= \begin{cases}-\left(x^{l} y^{k}+x^{l-1} y^{k+1}+\cdots+x^{k+1} y^{l-1}\right), & k<l, \\ 0, & k=l, \\ x^{k} y^{l}+x^{k-1} y^{l+1}+\cdots+x^{l+1} y^{k-1}, & k>l .\end{cases}
$$


Shortly,

$$
\begin{gathered}
\mathfrak{b}^{(0)}\left(x^{k} y^{l}\right)=\theta(k-l) \sum_{s=0}^{k-l-1} x^{l+s} y^{k-s-1}-\theta(l-k) \sum_{s=0}^{l-k-1} x^{k+s} y^{l-s-1} \\
\mathfrak{b}\left(x^{k} y^{l}\right)=\theta(k-l) \sum_{s=1}^{k-l} x^{l+s} y^{k-s}-\theta(l-k) \sum_{s=1}^{l-k} x^{k+s} y^{l-s}
\end{gathered}
$$

We list several useful matrix forms of the operators $\mathfrak{b}^{(0)}$ and $\mathfrak{b}$ in the basis formed by monomials, $e_{a} \otimes e_{b}:=x^{a} y^{b}$; for the operator $\mathfrak{b}^{(0)}$ :

$$
\begin{aligned}
\mathfrak{b}^{(0)} & =\sum_{i, j, a, b} \theta(j-a) \theta(j-b) \delta_{a+b+1}^{i+j} e_{a}^{j} \wedge e_{b}^{i} \\
& =\sum_{i, j, a, b}(\theta(j-a) \theta(j-b)-\theta(i-b) \theta(i-a)) \delta_{a+b+1}^{i+j} e_{a}^{j} \otimes e_{b}^{i} \\
& =\sum_{i, j: i<j} \sum_{a=1}^{j-i} e_{i+a-1}^{j} \wedge e_{j-a}^{i}
\end{aligned}
$$

and for the operator $\mathfrak{b}$ :

$$
\begin{aligned}
\mathfrak{b} & =\sum_{i, j, a, b} \theta(j+1-a) \theta(a-i) \delta_{a+b}^{i+j}\left(e_{a}^{j} \otimes e_{b}^{i}-e_{a}^{i} \otimes e_{b}^{j}\right) \\
& =\sum_{i, j, a, b}(\theta(j+1-a) \theta(a-i)-\theta(i+1-a) \theta(a-j)) \delta_{a+b}^{i+j} e_{a}^{j} \otimes e_{b}^{i} \\
& =\sum_{i, j: i<j} \sum_{a=1}^{j-i}\left(e_{i+a}^{j} \otimes e_{j-a}^{i}-e_{i+a}^{i} \otimes e_{j-a}^{j}\right) \\
& =\sum_{i, j: i<j}\left(\sum_{a=1}^{j-i-1} e_{i+a}^{j} \wedge e_{j-a}^{i}+e_{j}^{j} \otimes e_{i}^{i}-e_{j}^{i} \otimes e_{i}^{j}\right)
\end{aligned}
$$

where $x \wedge y=x \otimes y-y \otimes x$.

The Rota-Baxter operator $\mathfrak{r}_{\mathfrak{b}^{(0)}}$ corresponding to $\mathfrak{b}^{(0)}$ reads

$$
\mathfrak{r}_{\mathfrak{b}^{(0)}}(A)_{j}^{i}=\theta(j-i) \sum_{s \geq 0} A_{j-s-1}^{i-s}-\theta(i+1-j) \sum_{s \geq 0} A_{j+s}^{i+s+1} .
$$

In the right-hand side of (5.49), the summations are over those $s \geq 0$ for which the corresponding matrix element in the sum makes sense; that is, 
the range of $s$ in the first sum is $s=0,1, \ldots, i-1$ and, in the second sum, $s=0,1, \ldots n-i-1$;

The Rota-Baxter operator $\mathfrak{r}_{\mathfrak{b}}$ corresponding to $\mathfrak{b}$ reads (with the same convention about the summation ranges)

$$
\mathfrak{r}_{\mathfrak{b}}(A)_{j}^{i}=\theta(j+1-i) \sum_{s \geq 0} A_{j-s-1}^{i-s-1}-\theta(i-j) \sum_{s \geq 0} A_{j+s}^{i+s} .
$$

Its weight is -1 .

For the operator $r^{(s)}$, given by equation (5.3), the corresponding Rota-Baxter operator $\mathfrak{r}^{(s)}$ is

$$
\mathfrak{r}^{(s)}(A)_{j}^{i}=\left\{\begin{array}{cc}
-\theta(j-i) A_{j}^{i}, & i \neq j \\
\sum_{s: s<i} A_{s}^{s}, & i=j .
\end{array}\right.
$$

Its weight is -1 .

We shall give also the Rota-Baxter operator for the Bézout $r$-matrix $\mathfrak{b}$ in the rime basis, that is, for the $r$-matrix (4.2); it has weight 1 (since $r_{12}+r_{21}=P-I$ for $r$ in (4.2)). The Rota-Baxter operator has the form

$$
\mathfrak{r}(A)_{j}^{i}=\left\{\begin{array}{cc}
\frac{\phi_{j}}{\phi_{j}-\phi_{i}}\left(A_{j}^{i}-A_{j}^{j}\right), & i \neq j, \\
\sum_{s: s \neq i} \frac{\phi_{i}}{\phi_{i}-\phi_{s}}\left(A_{s}^{i}-A_{s}^{s}\right), & i=j .
\end{array}\right.
$$

\section{$5.5 *$-multiplication}

1. Let $\mathfrak{r}: \mathcal{A} \rightarrow \mathcal{A}$ be a Rota-Baxter operator of weight $\alpha$ (see equation (5.39)) on an algebra $\mathcal{A}$. It is known that the operation

$$
A * B:=\mathfrak{r}(A) B+A \mathfrak{r}(B)-\alpha A B, \quad A, B \in \mathcal{A},
$$

defines an associative product on the space $\mathcal{A}$. This product is closely related to the coproducts (5.33) and (5.34) by duality. We shall illustrate it in the context of the matrix algebras. 
Define an operation $\tilde{*}$ by

$$
\langle\tilde{\delta}(u), B \otimes A\rangle=\langle u, A \tilde{*} B\rangle
$$

where $\tilde{\delta}$ is given by (5.34). We have then

$$
\begin{aligned}
\langle\tilde{\delta}(u), B \otimes A\rangle= & \operatorname{Tr}_{12}\left(u_{1} r B_{1} A_{2}-r u_{2} B_{1} A_{2}+c u_{2} B_{1} A_{2}\right) \\
= & \operatorname{Tr}_{1}\left(u_{1} \operatorname{Tr}_{2}\left(r A_{2}\right) B_{1}\right)-\operatorname{Tr}_{1}\left(u_{1} A_{1} \operatorname{Tr}_{2}\left(B_{2} r_{21}\right)\right) \\
& +\operatorname{Tr}_{1}\left(c u_{1} \operatorname{Tr}(B) A_{1}\right) \\
= & \operatorname{Tr}\left(u\left[\mathfrak{r}(A) B-A \mathfrak{r}^{\prime}(B)+c A \operatorname{Tr}(B)\right]\right),
\end{aligned}
$$

thus

$$
A \tilde{*} B=\mathfrak{r}(A) B-A \mathfrak{r}^{\prime}(B)+c A \operatorname{Tr}(B) .
$$

In equation (5.55), $x_{i}$ stands for the copy of an element $x$ in the space number $i$ in $\mathcal{A} \otimes \mathcal{A}$; the operators $\mathfrak{r}$ and $\mathfrak{r}^{\prime}$ are given by (5.40); to obtain the second and the third terms in the second line of (5.55) we renumbered $1 \leftrightarrow 2$ and then moved $r$ cyclically under the trace in the second term.

Assume, as before, that $r_{12}+r_{21}=\alpha P_{12}+\beta I$ and $c=\beta$. Then, expressing $\mathfrak{r}^{\prime}(A)$ by (5.42), we find that the term with $\operatorname{Tr}(B)$ drops out and it follows that

$$
A \tilde{*} B=A * B
$$

2. We shall describe the $*$-multiplication in the simplest example of the Rota-Baxter operators (5.49) and (5.50) corresponding to the Bézout operators for the the polynomials of degree less than 2 (that is, for $2 \times 2$ matrices $\left.A=\left(\begin{array}{ll}a_{1}^{1} & a_{2}^{1} \\ a_{1}^{2} & a_{2}^{2}\end{array}\right) \equiv a_{j}^{i} e_{i}^{j}\right)$.

For the operator $\mathfrak{b}^{(0)}=e_{1}^{2} \wedge e_{1}^{1}$, we have

$$
\mathfrak{r}_{\mathfrak{b}^{(0)}}(A)=\left(\begin{array}{cc}
-a_{1}^{2} & a_{1}^{1} \\
0 & 0
\end{array}\right)
$$


and the $*$-multiplication reads

$$
A *^{o} \tilde{A} \equiv A \mathfrak{r}_{\mathfrak{b}^{(0)}}(\tilde{A})+\mathfrak{r}_{\mathfrak{b}(0)}(A) \tilde{A}=\left(\begin{array}{cc}
-a_{1}^{2} \tilde{a}_{1}^{1} & -a_{1}^{2} \tilde{a}_{2}^{1}+a_{1}^{1}\left(\tilde{a}_{1}^{1}+\tilde{a}_{2}^{2}\right) \\
-a_{1}^{2} \tilde{a}_{1}^{2} & a_{1}^{2} \tilde{a}_{1}^{1}
\end{array}\right)
$$

This algebra is isomorphic to the algebra of $3 \times 3$ matrices of the form

$$
\left(\begin{array}{ccc}
* & * & * \\
0 & 0 & * \\
0 & 0 & 0
\end{array}\right)
$$

with the identification

$$
\begin{array}{ll}
e_{1}^{1} \mapsto\left(\begin{array}{ccc}
0 & 1 & 0 \\
0 & 0 & 1 \\
0 & 0 & 0
\end{array}\right), & e_{2}^{1} \mapsto\left(\begin{array}{ccc}
-1 & 0 & 0 \\
0 & 0 & 0 \\
0 & 0 & 0
\end{array}\right), \\
e_{1}^{2} \mapsto\left(\begin{array}{ccc}
0 & 0 & 1 \\
0 & 0 & 0 \\
0 & 0 & 0
\end{array}\right), & e_{2}^{2} \mapsto\left(\begin{array}{ccc}
0 & 0 & 0 \\
0 & 0 & 1 \\
0 & 0 & 0
\end{array}\right) .
\end{array}
$$

For the operator $\mathfrak{b}=e_{2}^{2} \otimes e_{1}^{1}-e_{2}^{1} \otimes e_{1}^{2}$, we have

$$
\mathfrak{r}_{\mathfrak{b}}(A)=\left(\begin{array}{cc}
0 & 0 \\
-a_{1}^{2} & a_{1}^{1}
\end{array}\right)
$$

and the $*$-multiplication reads

$$
A * \tilde{A} \equiv A \mathfrak{r}_{\mathfrak{b}}(\tilde{A})+\mathfrak{r}_{\mathfrak{b}}(A) \tilde{A}+A \tilde{A}=\left(\begin{array}{cc}
a_{1}^{1} \tilde{a}_{1}^{1} & a_{1}^{1} \tilde{a}_{2}^{1}+a_{2}^{1}\left(\tilde{a}_{1}^{1}+\tilde{a}_{2}^{2}\right) \\
a_{1}^{1} \tilde{a}_{1}^{2} & a_{1}^{1} \tilde{a}_{2}^{2}+a_{2}^{2}\left(\tilde{a}_{1}^{1}+\tilde{a}_{2}^{2}\right)
\end{array}\right)
$$

This algebra is isomorphic to the algebra of $3 \times 3$ matrices of the form

$$
\left(\begin{array}{lll}
* & * & * \\
0 & * & 0 \\
0 & 0 & 0
\end{array}\right)
$$


with the identification

$$
\begin{array}{ll}
e_{1}^{1} \mapsto\left(\begin{array}{ccc}
1 & 0 & 0 \\
0 & 1 & 0 \\
0 & 0 & 0
\end{array}\right), & e_{2}^{1} \mapsto\left(\begin{array}{ccc}
0 & 0 & 1 \\
0 & 0 & 0 \\
0 & 0 & 0
\end{array}\right), \\
e_{1}^{2} \mapsto\left(\begin{array}{ccc}
0 & 1 & 0 \\
0 & 0 & 0 \\
0 & 0 & 0
\end{array}\right), \quad e_{2}^{2} \mapsto\left(\begin{array}{ccc}
0 & 0 & 0 \\
0 & 1 & 0 \\
0 & 0 & 0
\end{array}\right) .
\end{array}
$$

\section{Rime Poisson brackets}

The Poisson brackets having the form

$$
\left\{x^{i}, x^{j}\right\}=f_{i j}\left(x^{i}, x^{j}\right), \quad i, j=1,2, \ldots, n,
$$

with some functions $f_{i j}$ of two variables, we shall call rime. In this section we study quadratic rime Poisson brackets,

$$
\left\{x^{i}, x^{j}\right\}=a_{i j}\left(x^{i}\right)^{2}-a_{j i}\left(x^{j}\right)^{2}+2 \nu_{i j} x^{i} x^{j}, \quad i, j=1,2, \ldots, n .
$$

We show that there is a three-dimensional pencil of such Poisson brackets and then find the invariance group and the normal form of each individual member of the pencil.

\subsection{Rime pencil}

In this subsection we establish that the quadratic rime Poisson brackets form a three-dimensional Poisson pencil.

The left-hand side of (6.2) contains a matrix $a_{i j}$ with zeros on the diagonal, $a_{i i}=0$, and an anti-symmetric matrix $\nu_{i j}, \nu_{i j}=-\nu_{j i}$. The Jacobi identity constraints these matrices to satisfy

$$
a_{i j} a_{j k}+a_{i k}\left(\nu_{i j}+\nu_{j k}\right)=0, \quad i \neq j \neq k \neq i
$$

We shall describe a general solution of equation (6.3) in the strict situation, that is, when all $a_{i j}$ and $\nu_{i j}$ are different from zero for $i \neq j$.

The left-hand side of $\nu_{i j}+\nu_{j k}=-a_{i j} a_{j k} / a_{i k}$ is anti-symmetric with respect to $(i, k)$, that is $\Upsilon_{i j} \Upsilon_{j k} \Upsilon_{k i}=1$ for $\Upsilon_{i j}=-a_{i j} / a_{j i}$, which readily 
implies the existence of a vector $\phi_{i}$ such that $\Upsilon_{i j}=\phi_{i}^{2} / \phi_{j}^{2}$. Therefore,

$$
a_{i k}=\phi_{i} c_{i k} \phi_{k}^{-1}
$$

where the matrix $c_{i j}$ is anti-symmetric, $c_{i j}=-c_{j i}$. Next, $2 \nu_{k i}=-\left(\nu_{i j}+\right.$ $\left.\nu_{j k}\right)+\left(\nu_{j k}+\nu_{k i}\right)+\left(\nu_{k i}+\nu_{i j}\right)$; using (6.3) to express each bracket in the right-hand side, we find

$$
\nu_{k i}=\frac{1}{2}\left(\frac{c_{i j} c_{k i}}{c_{j k}}+\frac{c_{j k} c_{k i}}{c_{i j}}-\frac{c_{i j} c_{j k}}{c_{k i}}\right) .
$$

The right-hand side of equation (6.5) does not depend on $j$ which imposes further restrictions on the matrix $c_{i j}$ when $n>3$. Writing the sum $\nu_{i j}+\nu_{j k}+\nu_{k l}+\nu_{l i}$ in two ways, as $\left(\nu_{i j}+\nu_{j k}\right)+\left(\nu_{k l}+\nu_{l i}\right)$ and as $\left(\nu_{j k}+\right.$ $\left.\nu_{k l}\right)+\left(\nu_{l i}+\nu_{i j}\right)$, and using (6.3) to express each bracket in terms of the matrix $c$, we obtain

$$
\frac{c_{i j} c_{j k}-c_{i l} c_{l k}}{c_{i k}}=\frac{c_{j k} c_{k l}-c_{j i} c_{i l}}{c_{j l}} .
$$

Replacing $j$ by $m$ in (6.5) gives the condition on the matrix $c$ :

$$
\frac{c_{i j} c_{k i}}{c_{j k}}+\frac{c_{j k} c_{k i}}{c_{i j}}-\frac{c_{i j} c_{j k}}{c_{k i}}=\frac{c_{i m} c_{k i}}{c_{m k}}+\frac{c_{m k} c_{k i}}{c_{i m}}-\frac{c_{i m} c_{m k}}{c_{k i}} .
$$

Using equation (6.6) to rewrite the combination $\frac{c_{i j} c_{j k}}{c_{k i}}-\frac{c_{i m} c_{m k}}{c_{k i}}$, we find

$$
\begin{aligned}
\left(c_{j k} c_{k m}-c_{j i} c_{i m}\right) \Psi_{i j k m} & =0, \quad \text { where } \\
\Psi_{i j k m} & =\left(\frac{1}{c_{j k} c_{i m}}+\frac{1}{c_{i j} c_{k m}}+\frac{1}{c_{k i} c_{j m}}\right) .
\end{aligned}
$$

The quantity $\Psi_{i j k m}$ is totally anti-symmetric with respect to its indices. Therefore, if $\Psi_{i j k m} \neq 0$ then the combinations $\left(c_{j k} c_{k m}-c_{j i} c_{i m}\right)$ vanish for all permutations of indices. This is however impossible: the system of three linear equations

$$
\begin{aligned}
& c_{i j} c_{j k}-c_{i m} c_{m k}=0, \\
& c_{i k} c_{k m}-c_{i j} c_{j m}=0, \\
& c_{i m} c_{m j}-c_{i k} c_{k j}=0
\end{aligned}
$$

for unknowns $\left\{c_{j k}, c_{k m}, c_{m j}\right\}$ has, by definition, a non-zero solution but the determinant of the system is different from zero. Thus the Pfaffian $\Psi_{i j k m}$ vanishes for each quadruple $\{i, j, k, m\}$; in other words, the coefficients of 
the matrix $1 / c_{i j}$ satisfy the Plücker relations; therefore the form $1 / c_{i j}$ is decomposable, $c_{i j}^{-1}=s_{i} t_{j}-s_{j} t_{i}$, for some vectors $\vec{s}$ and $\vec{t}$. For each $i$, at least one of $s_{i}$ or $t_{i}$ is different from zero. Making, if necessary, a change of basis in the two-dimensional plane spanned by $\vec{s}$ and $\vec{t}$, we can therefore always assume that all components of, say, the vector $\vec{s}$ are different from zero, $s_{i} \neq 0 \forall i$. We represent the bivector $1 / c_{i j}$ in the form $\left(u_{i}^{-1}=s_{i}\right.$ and $\left.\psi_{i}=-t_{i} / s_{i}\right)$

$$
\frac{1}{c_{i j}}=u_{i}^{-1} u_{j}^{-1}\left(\psi_{i}-\psi_{j}\right)
$$

Substituting (6.10) into (6.5) we obtain

$$
\nu_{k i}+\frac{1}{2} \frac{u_{k}^{2}+u_{i}^{2}}{\psi_{k}-\psi_{i}}=-\frac{1}{2}\left(\frac{u_{i}^{2}-u_{j}^{2}}{\psi_{i}-\psi_{j}}-\frac{u_{k}^{2}-u_{j}^{2}}{\psi_{k}-\psi_{j}}\right) .
$$

Replacing $j$ by $m$ in the right-hand side and equating the resulting expressions, we find that the independency of the right-hand side on $j$ implies:

$$
\begin{aligned}
E_{i j k m}:= & \frac{u_{i}^{2}}{\left(\psi_{i}-\psi_{j}\right)\left(\psi_{i}-\psi_{k}\right)\left(\psi_{i}-\psi_{m}\right)} \\
& +\frac{u_{j}^{2}}{\left(\psi_{j}-\psi_{i}\right)\left(\psi_{j}-\psi_{k}\right)\left(\psi_{j}-\psi_{m}\right)} \\
& +\frac{u_{k}^{2}}{\left(\psi_{k}-\psi_{i}\right)\left(\psi_{k}-\psi_{j}\right)\left(\psi_{k}-\psi_{m}\right)} \\
& +\frac{u_{m}^{2}}{\left(\psi_{m}-\psi_{i}\right)\left(\psi_{m}-\psi_{j}\right)\left(\psi_{m}-\psi_{k}\right)}=0
\end{aligned}
$$

for every quadruple $\{i, j, k, m\}$.

The quantity $E_{i j k m}$ is totally symmetric. Selecting three values of the index, say, 1,2 and 3 , we can form the quadruple $\{i, 1,2,3\}$ for each $i$. Solving $E_{i 123}=0$, we obtain the following expression for $u_{i}^{2}$ :

$$
\begin{aligned}
u_{i}^{2}= & A_{1} \frac{\left(\psi_{i}-M_{2}\right)\left(\psi_{i}-M_{3}\right)}{\left(M_{1}-M_{2}\right)\left(M_{1}-M_{3}\right)}+A_{2} \frac{\left(\psi_{i}-M_{1}\right)\left(\psi_{i}-M_{3}\right)}{\left(M_{2}-M_{1}\right)\left(M_{2}-M_{3}\right)} \\
& +A_{3} \frac{\left(\psi_{i}-M_{1}\right)\left(\psi_{i}-M_{2}\right)}{\left(M_{3}-M_{1}\right)\left(M_{3}-M_{2}\right)}
\end{aligned}
$$

for some constants $A_{1}, A_{2}, A_{3}, M_{1}, M_{2}$ and $M_{3}$. The right-hand side is the value, at the point $\psi_{i}$, of a quadratic polynomial which equals to $A_{a}$ at the 
points $M_{a}, a=1,2,3$. Since $A_{1}, A_{2}, A_{3}, M_{1}, M_{2}$ and $M_{3}$ are arbitrary, we can simply write

$$
u_{i}^{2}=a \psi_{i}^{2}+b \psi_{i}+c
$$

With the expressions (6.14) for $u_{i}^{2}$, the equalities (6.12) are identically satisfied which shows that (6.14) is the general solution.

Upon rescaling $x^{i} \mapsto \phi_{i} u_{i} x^{i}$ with $\phi_{i}$ from (6.4), the Poisson brackets (6.2) simplify. The following statement is established (for $n=2$ or 3 , (6.14) does not impose a restriction on the anti-symmetric matrix $c_{i j}$ with all off-diagonal entries different from zero).

Proposition 6.1. Up to a rescaling of variables, the general strict quadratic rime Poisson brackets have the form

$$
\left\{x^{i}, x^{j}\right\}=\frac{\varrho\left(\psi_{j}\right)\left(x^{i}\right)^{2}+\varrho\left(\psi_{i}\right)\left(x^{j}\right)^{2}}{\psi_{i}-\psi_{j}}+\left(\left(\psi_{i}-\psi_{j}\right) a-\frac{\varrho\left(\psi_{i}\right)+\varrho\left(\psi_{j}\right)}{\psi_{i}-\psi_{j}}\right) x^{i} x^{j},
$$

where $\vec{\psi}$ is an arbitrary vector with pairwise distinct components and $\varrho(t)=a t^{2}+b t+c$ is an arbitrary quadratic polynomial ${ }^{6}$.

Thus the strict quadratic rime Poisson brackets form the threedimensional pencil (parameterized by the polynomial $\varrho$ ).

The Poisson brackets (6.15) can be rewritten in the following forms:

$$
\begin{aligned}
\left\{x^{i}, x^{j}\right\}= & \frac{1}{\psi_{i}-\psi_{j}}\left(\varrho\left(\psi_{j}\right) x^{i}-\varrho\left(\psi_{i}\right) x^{j}\right)\left(x^{i}-x^{j}\right) \\
& +a\left(\psi_{i}-\psi_{j}\right) x^{i} x^{j}, \\
\left\{x^{i}, x^{j}\right\}= & \frac{a u_{i j}^{2}+b u_{i j} v_{i j}+c v_{i j}^{2}}{\psi_{i}-\psi_{j}} \equiv \frac{v_{i j}^{2}}{\psi_{i}-\psi_{j}} \varrho\left(\frac{u_{i j}}{v_{i j}}\right),
\end{aligned}
$$

where $u_{i j}=\psi_{j} x^{i}-\psi_{i} x^{j}$ and $v_{i j}=x^{i}-x^{j}$.

Remark 6.1. For $\varrho(t)=b t$ (respectively, $\varrho(t)=c$ ) these Poisson brackets appear in the classical limit of the commutation relations (1.31) in the non-unitary (respectively, unitary) case (with the parameterization $\beta_{i j}=-\frac{\beta \psi_{j}}{\psi_{i}-\psi_{j}}$ in the non-unitary case).

\footnotetext{
${ }^{6}$ To have non-vanishing coefficients in the formula (6.15) one has to impose certain inequalities for the components of the vector $\vec{\psi}$ and the coefficients of the polynomial $\varrho$; however, the formula (6.15) defines Poisson brackets without these inequalities.
} 
Remark 6.2. The strict rime linear Poisson brackets

$$
\left\{x^{i}, x^{j}\right\}=a_{i j} x^{i}-a_{j i} x^{j}, \quad a_{i j} \neq 0 \quad \text { for all } i, j=1,2, \ldots, n: \quad i \neq j
$$

(or strict rime Lie algebras) are less interesting. The Jacobi identity is

$$
a_{i k} a_{k j}=a_{i j} a_{j k} \quad \text { for all } i \neq j \neq k \neq i \text {. }
$$

Rescale variables $x^{2}, x^{3}, \ldots, x^{n}$ to have $a_{1 i}=1, i=2, \ldots, n$. Then the condition (6.19) with one of $i, j, k$ equal 1 implies $a_{i j}=a_{j i}$ and $a_{i j}=a_{i 1} / a_{j 1}$, $i, j=2, \ldots, n$; it follows that $a_{i 1}^{2}=a_{j 1}^{2}, i, j=2, \ldots, n$. For $n>3$, the condition (6.19) with $i, j, k>1$ forces $a_{i 1}=a_{j 1}, i, j=2, \ldots, n$. Denote by $\nu$ this common value, $a_{i 1}=\nu, i, j=2, \ldots, n$. After a rescaling $x^{1} \mapsto \nu x^{1}$ we find a unique strict rime Lie algebra, $\left[x^{i}, x^{j}\right]=x^{i}-x^{j}$ for all $i$ and $k$, which is almost trivial: $\left[x^{i}, x^{k}-x^{l}\right]=-\left(x^{k}-x^{l}\right)$ for all $i, k$ and $l$ and $\left[x^{i}-x^{j}, x^{k}-x^{l}\right]=0$ for all $i, j, k, l$.

For $n=3$, there is one more possibility: $a_{31}=-a_{21}$. After a rescaling $x^{1} \mapsto a_{21} x^{1}$, the solution reads

$$
\left[x^{1}, x^{2}\right]=x^{1}-x^{2}, \quad\left[x^{1}, x^{3}\right]=x^{1}+x^{3}, \quad\left[x^{2}, x^{3}\right]=-x^{2}+x^{3} .
$$

This Lie algebra is isomorphic to $\mathfrak{s l}(2)$; the isomorphism is given, for example, by $h \mapsto x^{1}-x^{3}, e \mapsto x^{1}+x^{3}$ and $f \mapsto x^{2}-\left(x^{1}+x^{3}\right) / 4$ (here $h, e$ and $f$ are the standard generators of $\mathfrak{s l}(2),[h, e]=2 e,[h, f]=-2 f$ and $[e, f]=h)$.

\subsection{Invariance}

In this subsection we analyze the invariance group of each individual member of the Poisson pencil from Proposition 6.1. We find that the Poisson brackets (6.15), with arbitrary (non-vanishing) $\varrho$, admit a non-trivial 1-parametric invariance group.

The transformation law of Poisson brackets $\left\{x^{i}, x^{j}\right\}=f^{i j}(x)$ under an infinitesimal change of variables, $\tilde{x}^{i}=x^{i}+\epsilon \varphi^{i}(x), \epsilon^{2}=0$, is $\left\{\tilde{x}^{i}, \tilde{x}^{j}\right\}=$ $f^{i j}(\tilde{x})+\epsilon \delta_{x} f^{i j}$, where $\delta_{x} f^{i j}=\left\{\varphi^{i}, x^{j}\right\}+\left\{x^{i}, \varphi^{j}\right\}+\varphi^{k} \partial_{k} f^{i j}$. For a linear 
infinitesimal transformation, $\varphi^{i}(x)=A_{j}^{i} x^{j}$, we have

$$
\delta_{x} f^{i j}=A_{k}^{i}\left\{x^{k}, x^{j}\right\}+A_{k}^{j}\left\{x^{i}, x^{k}\right\}-x^{l} A_{l}^{k} \partial_{k}\left\{x^{i}, x^{j}\right\} .
$$

Specializing to the Poisson brackets (6.15), we find

$$
\delta_{x} f^{i j}=U_{j i}-U_{i j}
$$

with

$$
\begin{aligned}
U_{i j}:= & \sum_{s}\left(2 A_{s}^{i} \frac{\varrho_{j}}{\psi_{i j}}+A_{s}^{j}\left(\psi_{i j} a-\frac{\varrho_{i}+\varrho_{j}}{\psi_{i j}}\right)\right) x^{i} x^{s} \\
& +\sum_{s: s \neq i} A_{s}^{j}\left(\frac{\varrho_{i}}{\psi_{s i}}\left(x^{s}\right)^{2}+\frac{\varrho_{s}}{\psi_{s i}}\left(x^{i}\right)^{2}+\left(\psi_{s i} a-\frac{\varrho_{s}+\varrho_{i}}{\psi_{s i}}\right) x^{i} x^{s}\right),
\end{aligned}
$$

where $\psi_{i j}=\psi_{i}-\psi_{j}$ and $\varrho_{s}=\varrho\left(\psi_{s}\right)$.

The Poisson brackets (6.15) remain rime under the infinitesimal linear transformation with the matrix $A$ if the coefficients in $\left(x^{s}\right)^{2}, x^{s} x^{i}$ and $x^{s} x^{j}$, $s \neq i, j$, in (6.22) vanish which gives the following system:

$$
\begin{gathered}
\left(x^{s}\right)^{2}, \quad s \neq i, j \Rightarrow A_{s}^{j} \frac{\varrho_{i}}{\psi_{s i}}-A_{s}^{i} \frac{\varrho_{j}}{\psi_{s j}}=0, \\
x^{i} x^{s}, \quad s \neq i, j \Rightarrow 2 A_{s}^{i} \frac{\varrho_{j}}{\psi_{i j}}+A_{s}^{j}\left(\psi_{s j} a-\frac{\varrho_{i}+\varrho_{j}}{\psi_{i j}}-\frac{\varrho_{s}+\varrho_{i}}{\psi_{s i}}\right)=0 .
\end{gathered}
$$

Equation (6.24) implies that $A_{k}^{l}=\nu_{k} \varrho_{l} / \psi_{l k}, l \neq k$, with arbitrary constants $\nu_{k}$. For a quadratic polynomial $\varrho$, this solves equation (6.25) as well. The coefficient in $x^{j} x^{s}$ vanishes due to the anti-symmetry.

The Poisson brackets (6.15) are invariant under the infinitesimal linear transformation with the matrix $A$ if, in addition to (6.24) and (6.25), the coefficients in $\left(x^{i}\right)^{2},\left(x^{j}\right)^{2}$ and $x^{i} x^{j}$ in (6.22) vanish which gives:

$$
\begin{gathered}
x^{i} x^{j} \Rightarrow \varrho_{j} A_{j}^{i}+\varrho_{i} A_{i}^{j}=0 \\
\left(x^{i}\right)^{2} \Rightarrow A_{i}^{i} \frac{\varrho_{j}}{\psi_{i j}}+A_{i}^{j}\left(\psi_{i j} a-\frac{\varrho_{i}+\varrho_{j}}{\psi_{i j}}\right)+\sum_{s: s \neq i} A_{s}^{j} \frac{\varrho_{s}}{\psi_{s i}}=0 .
\end{gathered}
$$

Equation (6.26) implies that $\nu_{k}$ are equal, $\nu_{k}=\nu$. The matrix $A$ is defined up to a multiplicative factor, so we can set $\nu$ to 1 . Since the Poisson brackets (6.15) are quadratic, a global rescaling leaves them invariant, so we can add to $A$ a matrix, proportional to the identity matrix and make $A$ traceless. 
The traceless condition, together with equation (6.27) determines the diagonal entries, $A_{i}^{i}=a(n-1) \psi_{i}+\frac{n-1}{2} b+\varrho_{i} \sum_{s: s \neq i} \frac{1}{\psi_{s i}}$. The coefficient in $\left(x^{j}\right)^{2}$ vanishes due to the anti-symmetry. We summarize the obtained results.

Proposition 6.2. (i) The infinitesimal linear transformation with the matrix A leaves the Poisson brackets (6.15) rime if and only if

$$
A_{k}^{l}=\frac{\nu_{k} \varrho_{l}}{\psi_{l k}}, \quad l \neq k
$$

with arbitrary constants $\nu_{k}$.

(ii) Up to a global rescaling of coordinates, the invariance group of the Poisson brackets (6.15) is 1-parametric, with a generator $\mathcal{A}$,

$$
\mathcal{A}(\varrho)_{j}^{i}=\frac{\varrho_{i}}{\psi_{i j}}, \quad i \neq j, \quad \text { and } \quad \mathcal{A}(\varrho)_{i}^{i}=\frac{n-1}{2} \varrho_{i}^{\prime}+\varrho_{i} \xi_{i}, \quad \xi_{i}:=\sum_{s: s \neq i} \frac{1}{\psi_{s i}}
$$

where $\varrho_{i}^{\prime}$ is the value of the derivative of the polynomial $\varrho$ at the point $\psi_{i}$.

Since the Poisson brackets transformed with the matrix (6.28) are still rime, it follows from Proposition 6.1 that they can be written, after an appropriate rescalings of coordinates, in the form (6.15). In other words, the variation $\delta_{x}$ can be compensated by a variation of $\psi$ 's and $\varrho$ and a diagonal transformation of the coordinates. We have

$$
-\delta_{x} f^{i j}=\delta^{(1)}+\delta^{(2)},
$$

where

$$
\delta^{(1)}=\frac{\varrho_{i} \varrho_{j}\left(x^{i}-x^{j}\right)^{2}}{\psi_{i j}^{2}}\left(\nu_{i}-\nu_{j}\right)+a\left(\nu_{j} \varrho_{j}\left(x^{i}\right)^{2}-\nu_{i} \varrho_{i}\left(x^{j}\right)^{2}\right)
$$

and

$$
\delta^{(2)}=\left(\tilde{A}_{i}^{i}-\tilde{A}_{j}^{j}\right) \frac{\varrho_{j}\left(x^{i}\right)^{2}-\varrho_{i}\left(x^{j}\right)^{2}}{\psi_{i j}}, \quad \tilde{A}_{i}^{i}:=A_{i}^{i}-\varrho_{i}^{\prime} \nu_{i}-\sum_{s: s \neq i} \frac{\nu_{s} \varrho_{s}}{\psi_{s i}} .
$$

Choose $A_{i}^{i}$ to set $\tilde{A}_{i}^{i}$ to 0 ; this is a diagonal transformation of the coordinates. Then $\delta^{(2)}$ vanishes and the variation of $f^{i j}$ is reduced to $\delta^{(1)}$. 
On the other hand, under a variation of $\psi^{\prime} s, \psi_{i} \mapsto \psi_{i}+\delta \psi_{i}$, the Poisson brackets (6.15) transform in the following way:

$$
\delta_{\psi} f^{i j}=\frac{\left(x^{i}-x^{j}\right)^{2}}{\psi_{i j}^{2}}\left(\varrho_{i} \delta \psi_{j}-\varrho_{j} \delta \psi_{i}\right)+a\left(\left(x^{j}\right)^{2} \delta \psi_{i}-\left(x^{i}\right)^{2} \delta \psi_{j}\right)
$$

and we conclude that with the choice

$$
\delta \psi_{i}=\epsilon \varrho_{i} \nu_{i}
$$

the variation $\delta^{(1)}$ is compensated by the variation $\delta_{\psi}$. The coefficients of the polynomial $\varrho$ stay the same. In the next subsection we will study relations between the variation of $\psi$ 's and the polynomial $\varrho$.

Remark. With $\xi_{i}$ as in (6.29), define three operators:

$$
\begin{gathered}
\left(B^{-}\right)_{j}^{i}=\frac{1}{\psi_{i j}}, \quad i \neq j \text { and }\left(B^{-}\right)_{i}^{i}=-\xi_{i}, \\
\left(B^{0}\right)_{j}^{i}=\frac{\psi_{i}}{\psi_{i j}}, \quad i \neq j \text { and }\left(B^{0}\right)_{i}^{i}=-\left(\frac{n-1}{2}+\psi_{i} \xi_{i}\right), \\
\left(B^{+}\right)_{j}^{i}=\frac{\psi_{i}^{2}}{\psi_{i j}}, \quad i \neq j \text { and }\left(B^{+}\right)_{i}^{i}=-\left((n-1) \psi_{i}+\psi_{i}^{2} \xi_{i}\right) .
\end{gathered}
$$

The operators $B^{+}, B^{0}$ and $B^{-}$generate an action of the Lie algebra $s l(2)$,

$$
\left[B^{0}, B^{-}\right]=-B^{-}, \quad\left[B^{0}, B^{+}\right]=B^{+}, \quad\left[B^{+}, B^{-}\right]=-2 B^{0}
$$

(to obtain the usual commutation relations for the generators of $\operatorname{sl}(2)$, change the sign of $B^{+}$).

This is the usual projective action of $\operatorname{sl}(2)$ on polynomials $f(t)$ of degree less than $n$,

$$
\begin{aligned}
& B^{-}: f(t) \mapsto f^{\prime}(t), \quad B^{0}: f(t) \mapsto t f^{\prime}(t)-\frac{n-1}{2} f(t), \\
& B^{+}: f(t) \mapsto t^{2} f^{\prime}(t)-(n-1) t f(t),
\end{aligned}
$$

written in the basis of the non-normalized Lagrange polynomials, $l_{i}(t)=$ $\prod_{s: s \neq i}\left(t-\psi_{s}\right)$, at points $\left\{\psi_{i}\right\}, i=1,2, \ldots, n$. Indeed, in the basis $\left\{l_{i}(t)\right\}$, a 
polynomial $f(t), \operatorname{deg}(f) \leq n-1$, takes the form $f=\sum f^{i} l_{i}$, where $f^{i}=l_{i}\left(\psi_{i}\right)^{-1} f\left(\psi_{i}\right)$. We have

$$
l_{i}^{\prime}(t)=\sum_{a: a \neq i} \prod_{b: b \neq a, i}\left(t-\psi_{b}\right), \quad \text { so } \quad l_{i}^{\prime}\left(\psi_{k}\right)=\prod_{b: b \neq k, i} \psi_{k b}=l_{k}\left(\psi_{k}\right) \frac{1}{\psi_{k i}}, \quad k \neq i .
$$

Also,

$$
l_{i}^{\prime}(t)=l_{i}(t) \sum_{s: s \neq i} \frac{1}{t-\psi_{s}}, \quad \text { so } \quad l_{i}^{\prime}\left(\psi_{i}\right)=-l_{i}\left(\psi_{i}\right) \xi_{i}
$$

Therefore, $l_{i}^{\prime}(t)=-\xi_{i} l_{i}(t)+\sum_{k: k \neq i} \frac{1}{\psi_{k i}} l_{k}(t)$, which is exactly (6.35). For functions on the set of points $\left\{\psi_{i}\right\}$, the operator of multiplication by $t$ acts as a diagonal matrix $\operatorname{Diag}\left(\psi_{1}, \psi_{2}, \ldots, \psi_{n}\right)$ and (6.36)-(6.37) follow.

Define an involution $\varpi$ on the space of matrices, ${ }^{7}$

$$
\varpi(Y)_{j}^{i}=Y_{j}^{i}, \quad i \neq j \text { and } \varpi(Y)_{i}^{i}=-Y_{i}^{i}, \quad Y \in \operatorname{Mat}_{\mathrm{n}} .
$$

Let

$$
B(\varrho)=a B^{+}+b B^{0}+c B^{-}, \quad B(\varrho): f \mapsto \varrho(t) f^{\prime}(t)-\frac{n-1}{2} \varrho^{\prime}(t) f(t) .
$$

In the basis $\left\{l_{i}(t)\right\}$ for $B$, the generator (6.29) of the invariance group is

$$
\mathcal{A}(\varrho)=\varpi(B(\varrho)) .
$$

Note that the operators $\varpi\left(B^{-}\right), \varpi\left(B^{0}\right)$ and $\varpi\left(B^{+}\right)$do not form a Lie algebra.

\footnotetext{
${ }^{7}$ The involution $\varpi$ is the difference of two complementary projectors. The involution $\varpi$ satisfies

$$
\varpi\left(Y_{1}\right) \varpi\left(Y_{2}\right)+\varpi\left(Y_{1} Y_{2}\right)=\left\{\begin{array}{l}
\varpi\left(\varpi\left(Y_{1}\right) \varpi\left(Y_{2}\right)\right)+Y_{1} Y_{2}, \\
\varpi\left(\varpi\left(Y_{1}\right) Y_{2}\right)+Y_{1} \varpi\left(Y_{2}\right), \\
\varpi\left(Y_{1} \varpi\left(Y_{2}\right)\right)+\varpi\left(Y_{1}\right) Y_{2}
\end{array}\right.
$$

for arbitrary $Y_{1}, Y_{2} \in \mathrm{Mat}_{n}$. All other linear dependencies between $Y_{1} Y_{2}, \varpi\left(Y_{1}\right) Y_{2}$, $Y_{1} \varpi\left(Y_{2}\right), \varpi\left(Y_{1}\right) \varpi\left(Y_{2}\right), \varpi\left(Y_{1} Y_{2}\right), \varpi\left(\varpi\left(Y_{1}\right) Y_{2}\right), \varpi\left(Y_{1} \varpi\left(Y_{2}\right)\right)$ and $\varpi\left(\varpi\left(Y_{1}\right) \varpi\left(Y_{2}\right)\right)$ are consequences of the three identities (6.42).
} 


\subsection{Normal form}

In this subsection we derive a normal form of each individual member of the Poisson pencil from Proposition 6.1. It depends only on the discriminant of the polynomial $\varrho$. When the discriminant of $\varrho$ is different from zero, the Poisson brackets (6.15) are equivalent to the Poisson brackets defined by the $r$-matrix (4.3). When the polynomial $\varrho$ is different from zero but its discriminant is zero, the Poisson brackets (6.15) are equivalent to the Poisson brackets defined by the $r$-matrix (4.27).

Under a variation of the polynomial $\varrho, \varrho(t) \mapsto(a+\delta a) t^{2}+(b+\delta b) t+$ $(c+\delta c)$, we have for the Poisson brackets $(6.17)$ :

$$
\delta_{\varrho} f^{i j}=\frac{u_{i j}^{2} \delta a+u_{i j} v_{i j} \delta b+v_{i j}^{2} \delta c}{\psi_{i}-\psi_{j}} .
$$

The variation of $\varrho$ can be compensated by a variation (6.33) of $\psi$ 's if the coefficients in $\left(x^{j}\right)^{2}, x^{i} x^{j}$ and $\left(x^{i}\right)^{2}$ in the combination $\left(\delta_{\psi}+\delta_{\varrho}\right) f^{i j}$ vanish, which gives the following system:

$$
\begin{gathered}
\left(x^{j}\right)^{2} \Rightarrow \frac{\varrho_{i} \delta \psi_{j}-\varrho_{j} \delta \psi_{i}}{\psi_{i j}^{2}}+a \delta \psi_{i}+\frac{\psi_{i}^{2} \delta a+\psi_{i} \delta b+\delta c}{\psi_{i j}}=0 \\
x^{i} x^{j} \Rightarrow-\frac{2\left(\varrho_{i} \delta \psi_{j}-\varrho_{j} \delta \psi_{i}\right)}{\psi_{i j}^{2}}-\frac{2 \psi_{i} \psi_{j} \delta a+\left(\psi_{i}+\psi_{j}\right) \delta b+2 \delta c}{\psi_{i j}}=0
\end{gathered}
$$

A combination $2 \times(6.47)+(6.48)$ gives

$$
2 a \delta \psi_{i}+2 \psi_{i} \delta a+\delta b=0
$$

Substituting the expression (6.49) for $\delta \psi$ 's into (6.47) gives

$$
\delta D(\varrho)=0 \quad \text { where } D(\varrho)=b^{2}-4 a c
$$

The coefficient in $\left(x^{i}\right)^{2}$ in $\left(\delta_{\psi}+\delta_{\varrho}\right) f^{i j}$ vanishes due to the anti-symmetry.

Therefore, a necessary condition for a variation of $\varrho$ to be compensated by a variation of $\psi$ 's is that the discriminant $D(\varrho)$ does not vary. We shall now see that the discriminant is the unique invariant.

Explicitly, under a shift $\psi_{j} \mapsto \psi_{j}+\zeta$, we have $u_{i j} \mapsto u_{i j}+\zeta v_{i j}$ and $v_{i j} \mapsto v_{i j}$ (in the notation (6.17)), which produces the following effect on the 
coefficients of the polynomial $\varrho$ :

$$
a \mapsto a, \quad b \mapsto b+2 \zeta a, \quad c \mapsto c+\zeta b+\zeta^{2} a .
$$

A dilatation $\psi_{j} \mapsto \lambda \psi_{j}$ produces the following effect on the coefficients of $\varrho$ :

$$
a \mapsto \lambda a, \quad b \mapsto b, \quad c \mapsto \lambda^{-1} c .
$$

The inversion $\psi_{j} \mapsto \psi_{j}^{-1}$ accompanied by a change of variables $\tilde{x}^{i}=\psi_{i}^{-1} x^{i}$ produces the following effect on the coefficients of $\varrho$ :

$$
a \mapsto-c, \quad b \mapsto-b, \quad c \mapsto-a .
$$

The set of operators (6.51) and (6.52) generates the action of the affine group on the space of the polynomials $\varrho$. The affine group, together with the inversion (6.53) generates an action ${ }^{8}$ of $s o(3)$ (the spin 1 representation of $s l(2))$ on the space of the polynomials $\varrho$ and the classification reduces to that of orbits. The orbits (in the complex situation) of non-zero polynomials are of two types: "massive", $D(\varrho) \neq 0$, or "light-like", $D(\varrho)=0$. Particular representatives of both types appear in the Poisson brackets, corresponding to the rime $r$-matrices (see Remark 1 after Proposition 6.1) and thus to the $r$-matrices studied in Subsections 4.1 and 4.3. We obtain the following statement.

Proposition 6.3. Let $\varrho(t)$ be a non-zero quadratic polynomial.

If the discriminant of $\varrho$ is different from zero, $D(\varrho) \neq 0$, then there exists a change of the parameters $\psi^{i}$ in the Poisson brackets (6.15) which sets $\varrho(t)$ to bt, $\varrho(t) \mapsto b t$; these are the Poisson brackets corresponding to the $r$-matrix $r_{\mathrm{CG}}$ (Subsection 4.1).

If the discriminant of $\varrho$ is zero, $D(\varrho)=0$, then there exists a change of the parameters $\psi^{i}$ in the Poisson brackets (6.15) which sets $\varrho(t)$ to $c$, $\varrho(t) \mapsto c$; these are the Poisson brackets corresponding to the r-matrix $b_{\mathrm{CG}}$ (Subsection 4.3).

The generator $\mathcal{A}(\varrho)$ of the invariance group can be easily described in both cases, $D(\varrho) \neq 0$ and $D(\varrho)=0$, in the parameter-free basis (that is, for the $r$-matrices $r_{\mathrm{CG}}$ and $b_{\mathrm{CG}}$; in the rime basis the generators are given by (2.19) and (2.22), respectively). For $D(\varrho) \neq 0$ (respectively, $D(\varrho)=0$ ), it coincides with the matrix of the operator $B_{0}$ (respectively, $B_{-}$), as in the remark in

\footnotetext{
${ }^{8}$ Let $e_{+}$be the generator of the 1-parametric group (6.51) and $h$ the generator of the 1 -parametric group (6.52). Denote by $\mathcal{I}$ the inversion (6.53). The remaining generator $e_{-}$is $\mathcal{I} e_{+} \mathcal{I}$.
} 
Subsection 6.2 , in the basis $\left\{t^{i}\right\}$ of powers of the variable $t$. This implies somewhat unexpectedly that for an arbitrary polynomial $\varrho(t)$ the matrices $\mathcal{A}(\varrho)$ and $\varpi(\mathcal{A}(\varrho))$ are related by a similarity transformation. Note that in the basis $\left\{t^{i}\right\}$ of powers, the operators $a B^{+}+b B^{0}+c B^{-}$and $\varpi\left(a B^{+}+\right.$ $\left.b B^{0}+c B^{-}\right)$are also related by a similarity transformation for arbitrary $a, b$ and $c$ but here it is obvious: $\varpi\left(a B^{+}+b B^{0}+c B^{-}\right)=a B^{+}-b B^{0}+c B^{-}$, so the operator $\varpi\left(a B^{+}+b B^{0}+c B^{-}\right)$belongs to $\operatorname{sl}(2)$ and moreover lies on the same (complex) orbit as $a B^{+}+b B^{0}+c B^{-}$with respect to the adjoint action.

\section{Orderable quadratic rime associative algebras}

Consider an associative algebra $\mathcal{A}$ defined by quadratic relations giving a lexicographical order. This means that $x^{j} x^{k}$ for $j<k$ is a linear combination of terms $x^{a} x^{b}$ with $a \geq b$ and either $a>j$ or $a=j$ and $b>k$.

We shall say that such algebra $\mathcal{A}$ is rime if $\{a, b\} \subset\{j, k\}$. In other words, the relations in the algebra are

$$
x^{j} x^{k}=f_{j k} x^{k} x^{j}+g_{j k} x^{k} x^{k}, \quad j<k .
$$

We shall classify the strict rime algebras $\mathcal{A}$ (that is, the algebras for which all coefficients $f_{i j}$ and $g_{i j}$ are different from zero for $i<j$ ).

The only possible overlaps for the set of relations (7.1) are of the form $\left(x^{j} x^{k}\right) x^{l}=x^{j}\left(x^{k} x^{l}\right), j<k<l$. The ordered form of the expression $\left(x^{j} x^{k}\right) x^{l}$ is

$$
\begin{aligned}
\left(x^{j} x^{k}\right) x^{l}= & f_{j k} f_{j l} f_{k l} x^{l} x^{k} x^{j}+f_{j k} f_{j l} g_{k l} x^{l} x^{l} x^{j}+f_{k l}^{2} g_{j k} x^{l} x^{k} x^{k} \\
& +\left(f_{k l} g_{j k} g_{k l}+f_{k l}^{2}\left(f_{j k} g_{j l}+g_{j k} g_{k l}\right)\right) x^{l} x^{l} x^{k} \\
& +\left(f_{j k} g_{j l} g_{k l}+g_{j k} g_{k l}^{2}+f_{k l} g_{k l}\left(f_{j k} g_{j l}+g_{j k} g_{k l}\right)\right) x^{l} x^{l} x^{l} .
\end{aligned}
$$

The ordered form of the expression $x^{j}\left(x^{k} x^{l}\right)$ is

$$
\begin{aligned}
x^{j}\left(x^{k} x^{l}\right)= & f_{j k} f_{j l} f_{k l} x^{l} x^{k} x^{j}+f_{j l}^{2} g_{k l} x^{l} x^{l} x^{j}+f_{k l} f_{j l} g_{j k} x^{l} x^{k} x^{k} \\
& +f_{k l} g_{j l} x^{l} x^{l} x^{k}+\left(g_{k l} g_{j l}+f_{j l} g_{k l} g_{j l}\right) x^{l} x^{l} x^{l} .
\end{aligned}
$$

Equating coefficients, we find

$$
\begin{gathered}
x^{l} x^{l} x^{j}: f_{j k} f_{j l} g_{k l}=f_{j l}^{2} g_{k l}, \\
x^{l} x^{k} x^{k}: f_{k l}^{2} g_{j k}=f_{k l} f_{j l} g_{j k},
\end{gathered}
$$




$$
\begin{aligned}
x^{l} x^{l} x^{k}: f_{k l} g_{j k} g_{k l}+f_{k l}^{2}\left(f_{j k} g_{j l}+g_{j k} g_{k l}\right) & =f_{k l} g_{j l}, \\
x^{l} x^{l} x^{l}: f_{j k} g_{j l} g_{k l}+g_{j k} g_{k l}^{2}+f_{k l} g_{k l}\left(f_{j k} g_{j l}+g_{j k} g_{k l}\right) & =g_{k l} g_{j l}+f_{j l} g_{k l} g_{j l} .
\end{aligned}
$$

In the strict situation, equations (7.4) and (7.5) simplify, respectively, to

$$
\begin{array}{ll}
f_{j k}=f_{j l} & \text { for } j<k \text { and } j<l, \\
f_{k l}=f_{j l} & \text { for } j<l \text { and } k<l .
\end{array}
$$

Equations (7.8) and (7.9) imply that $f_{j k}$ 's are all equal,

$$
f_{j k}=: f .
$$

The substitution of (7.10) into (7.6) gives (in the strict situation)

$$
(f+1)\left(g_{j k} g_{k l}+g_{j l}(f-1)\right)=0 \quad \text { for } j<k<l .
$$

Equation (7.7) follows from (7.10) and (7.11).

We have thus two cases:

(i) $f=-1$ and no extra conditions on $g_{j k}$ 's;

(ii) $f \neq-1$ and

$$
g_{j k} g_{k l}=(1-f) g_{j l} \quad \text { for } j<k<l \text {; }
$$

$1-f \neq 0$ since $g_{j k} \neq 0$ and $g_{k l} \neq 0$.

In the case (ii), make an appropriate rescaling of generators, $x^{i} \mapsto d_{i} x^{i}$ to achieve

$$
g_{i, i+1}=1-f \quad \text { for all } i=1, \ldots, n-1 .
$$

It then follows from equation (7.12) that

$$
g_{i j}=1-f \quad \text { for all } i<j .
$$

We summarize the obtained results.

Proposition 7.1. Up to a rescaling of variables, the general orderable quadratic strict rime algebra has relations 
(i) either of the form

$$
x^{j} x^{k}=-x^{k} x^{j}+g_{j k} x^{k} x^{k}, \quad j<k,
$$

with no conditions on the coefficients $g_{j k}$;

(ii) or of the form

$$
x^{j} x^{k}=f x^{k} x^{j}+(1-f) x^{k} x^{k}, \quad j<k,
$$

with arbitrary $f$ (it is strict when $f \neq 0,1$ ).

By construction, the algebras of types (i) and (ii) possess a basis formed by ordered monomials and thus have the Poincaré series of the algebra of commuting variables.

The algebra with defining relations (7.16) is the quantum space for the $R$-matrix (3.20). The relations (7.16) can be written in the form

$$
\left(x^{j}-x^{k}\right) x^{k}=f x^{k}\left(x^{j}-x^{k}\right), \quad j<k ;
$$

this is a quantization of the Poisson brackets

$$
\left\{x^{j}, x^{k}\right\}=x^{k}\left(x^{j}-x^{k}\right), \quad j<k .
$$

It would be interesting to know if the algebra with the defining relations (7.15) admits an $R$-matrix description.

\section{Acknowledgments}

It is our pleasure to thank László Fehér, Alexei Isaev and Milen Yakimov for enlightening discussions. The work was partially supported by the ANR project GIMP No ANR-05-BLAN-0029-01. The second author (T. Popov) was also partially supported by the Program "Bourses d'échanges scientifiques pour les pays de l'Est européen" and by the Bulgarian National Council for Scientific Research project PH-1406. 


\section{Appendix A. Equations}

Here we give the list of the equations $Y B(\hat{R})_{a b c}^{i j k}=0$ for the rime matrix

$$
\hat{R}_{k l}^{i j}=\alpha_{i j} \delta_{l}^{i} \delta_{k}^{j}+\beta_{i j} \delta_{k}^{i} \delta_{l}^{j}+\gamma_{i j} \delta_{k}^{i} \delta_{l}^{i}+\gamma_{i j}^{\prime} \delta_{k}^{j} \delta_{l}^{j}
$$

with a convention $\alpha_{i}=\alpha_{i i}$ and $\beta_{i i}=\gamma_{i i}=\gamma_{i i}^{\prime}=0$.

The rime Ansatz implies that $Y B(\hat{R})_{a b c}^{i j k}$ can be different from zero only if the set of lower indices is contained in the set of upper indices. Therefore, the equations split into two lists: the first one with two different indices among $\{i, j, k\}$ and the second one with three different indices.

The full set of equations $Y B(\hat{R})_{a b c}^{i j k}=0$ is invariant under the involution $\iota$,

$$
\iota: \alpha_{i} \leftrightarrow \alpha_{i}, \quad \alpha_{i j} \leftrightarrow \alpha_{j i}, \quad \beta_{i j} \leftrightarrow \beta_{j i}, \quad \gamma_{i j} \leftrightarrow \gamma_{j i}^{\prime}
$$

for if $\hat{R}$ is a solution of the YBe then $\hat{R}_{21}=P \hat{R} P$ is a solution of the YBe as well. We shall write only the necessary part of the equations, the rest can be obtained by the involution $\iota$.

The equations $Y B(\hat{R})_{a b c}^{i j k}=0$ with two different indices are:

$$
\begin{gathered}
\alpha_{i j} \gamma_{i j}\left(\gamma_{j i}+\gamma_{i j}^{\prime}\right)=0 \\
\alpha_{i j}\left(\beta_{i j} \beta_{j i}+\gamma_{i j} \gamma_{i j}^{\prime}\right)=0=\alpha_{i j}\left(\beta_{i j} \beta_{j i}-\gamma_{i j} \gamma_{j i}\right), \\
\alpha_{i j} \gamma_{i j}\left(\alpha_{i j}+\beta_{j i}-\alpha_{j}\right)=0=\alpha_{i j} \gamma_{i j}\left(\alpha_{j i}+\beta_{i j}-\alpha_{j}\right), \\
\beta_{i j}\left(\alpha_{i}^{2}-\alpha_{i j} \alpha_{j i}-\alpha_{i} \beta_{i j}\right)+\left(\alpha_{i}-\beta_{i j}\right) \gamma_{i j} \gamma_{i j}^{\prime}=0, \\
\left(\alpha_{i}-\alpha_{j}\right) \gamma_{i j}^{2}+\alpha_{i j} \gamma_{i j}\left(\gamma_{i j}+\gamma_{j i}^{\prime}\right)=0, \\
\alpha_{i j} \beta_{i j} \gamma_{j i}^{\prime}+\left(\alpha_{i} \beta_{i j}+\gamma_{i j}^{\prime} \gamma_{i j}\right) \gamma_{i j}=0 \\
\left(\alpha_{i j}-\alpha_{j i}-\beta_{i j}+\beta_{j i}\right) \gamma_{i j} \gamma_{j i}^{\prime}=0=\left(\alpha_{i j}-\alpha_{j i}-\beta_{i j}+\beta_{j i}\right) \beta_{i j} \beta_{j i} \\
\alpha_{i j} \gamma_{j i}^{\prime}\left(\alpha_{j}-\alpha_{i j}\right)+\beta_{j i} \gamma_{i j}\left(\alpha_{i}-\beta_{j i}\right)+\gamma_{i j}\left(\beta_{i j} \beta_{j i}+\gamma_{j i} \gamma_{j i}^{\prime}\right)=0, \\
\left(\alpha_{i}^{2}-\alpha_{i}\left(\alpha_{j i}+\beta_{j i}\right)+\beta_{i j} \beta_{j i}-\gamma_{i j} \gamma_{j i}\right) \gamma_{i j} \\
=\left(\alpha_{i}^{2}-\alpha_{i}\left(\alpha_{i j}+\beta_{i j}\right)+\beta_{i j} \beta_{j i}-\gamma_{i j}^{\prime} \gamma_{j i}^{\prime}\right) \gamma_{j i}^{\prime} .
\end{gathered}
$$

The equations with three different indices $\{i, j, k\}$ are:

$$
\begin{gathered}
\left(\alpha_{i j}-\alpha_{k i}-\beta_{i j}+\beta_{k i}\right) \gamma_{i j} \gamma_{k i}^{\prime}=0, \\
\alpha_{i j}\left(\beta_{i j} \beta_{j k}+\beta_{i k} \beta_{j i}-\beta_{i k} \beta_{j k}\right)=0, \\
\alpha_{i j}\left(\gamma_{i j} \gamma_{j k}+\gamma_{i k}\left(\beta_{j k}-\beta_{j i}\right)\right)=\alpha_{i j}\left(\gamma_{i j} \gamma_{k j}^{\prime}+\gamma_{i k}\left(\beta_{k j}-\beta_{i j}\right)\right)=0,
\end{gathered}
$$




$$
\begin{gathered}
\left(\alpha_{i j} \alpha_{j i}-\alpha_{j k} \alpha_{k j}\right) \beta_{i k}+\beta_{i j} \beta_{j k}\left(\beta_{i j}-\beta_{j k}\right)=0, \\
\left(\alpha_{i}+\beta_{i k}-\beta_{j i}\right) \beta_{j i} \gamma_{i k}+\gamma_{i k} \gamma_{j i} \gamma_{j i}^{\prime}+\alpha_{i k}\left(\gamma_{j k} \gamma_{j i}^{\prime}+\beta_{j k} \gamma_{k i}^{\prime}\right)=0, \\
\left(\alpha_{i}+\alpha_{i j}-\alpha_{k j}-\beta_{k j}\right) \gamma_{i j} \gamma_{i k}-\gamma_{i k}^{2} \gamma_{k j}+\gamma_{i j}\left(\alpha_{i k} \gamma_{k i}^{\prime}-\gamma_{i j} \gamma_{k j}^{\prime}\right)=0, \\
\left(\alpha_{i}-\beta_{k j}\right) \beta_{i j} \gamma_{i k}+\left(\beta_{i k} \beta_{k j}+\gamma_{i j} \gamma_{i j}^{\prime}\right) \gamma_{i k}+\alpha_{i k} \beta_{i j} \gamma_{k i}^{\prime}-\left(\beta_{i j}-\beta_{i k}\right) \gamma_{i j} \gamma_{k j}^{\prime}=0 \\
\alpha_{i j}\left(\gamma_{i j} \gamma_{j k}+\gamma_{i k}\left(\alpha_{j k}-\alpha_{j i}\right)\right)=\alpha_{j i}\left(\gamma_{i j} \gamma_{j k}+\gamma_{i k}\left(\alpha_{j k}-\alpha_{j i}\right)\right) \\
=\alpha_{i j}\left(\gamma_{i j} \gamma_{k j}^{\prime}+\gamma_{i k}\left(\alpha_{k j}-\alpha_{i j}\right)\right)=0 .
\end{gathered}
$$

\section{Appendix B. Blocks}

We analyze here the structure of $4 \times 4$ blocks of an invertible and skewinvertible rime $R$-matrix corresponding to two-dimensional coordinate planes.

We denote the matrix elements as in (1.5).

The skew-invertibility of a rime $R$-matrix imposes restrictions on its entries: in the line $\hat{R}_{j *}^{i *}$ only two entries can be non-zero, $\hat{R}_{j i}^{i j}$ and $\hat{R}_{j j}^{i j}$; in the line $\hat{R}_{* i}^{* j}$ only two entries can be non-zero, $\hat{R}_{j i}^{i j}$ and $\hat{R}_{i i}^{i j}$. Therefore,

$$
\alpha_{i j}=0 \Rightarrow \gamma_{i j} \gamma_{i j}^{\prime} \neq 0 \quad \text { and } \quad \gamma_{i j} \gamma_{i j}^{\prime}=0 \Rightarrow \alpha_{i j} \neq 0
$$

Dealing with a single block, this becomes especially clear: to skew invert a $4 \times 4$ block is the same as to invert the matrix

$$
\left(\begin{array}{cccc}
\alpha_{1} & 0 & \gamma_{12} & \beta_{12} \\
0 & 0 & \alpha_{12} & \gamma_{12}^{\prime} \\
\gamma_{21}^{\prime} & \alpha_{21} & 0 & 0 \\
\beta_{21} & \gamma_{21} & 0 & \alpha_{2}
\end{array}\right)
$$

whose determinant is

$$
\left(\alpha_{12} \beta_{12}-\gamma_{12} \gamma_{12}^{\prime}\right)\left(\alpha_{21} \beta_{21}-\gamma_{21} \gamma_{21}^{\prime}\right)-\alpha_{1} \alpha_{2} \alpha_{12} \alpha_{21}
$$

\section{B.1 Solutions}

Here we classify solutions which are neither ice nor strict rime. For an ice $R$-matrix, $\alpha_{12} \neq 0$ and $\alpha_{21} \neq 0$. For a rime $R$-matrix, $\alpha_{i j}$ might vanish and we consider the cases according to the number of $\alpha_{i j}$ 's which can be zero. 
1. Both $\alpha_{12}$ and $\alpha_{21}$ do not vanish, $\alpha_{12} \alpha_{21} \neq 0$.

If $\gamma_{12} \gamma_{21} \neq 0$ then by (A.3), $\gamma_{12}^{\prime} \gamma_{21}^{\prime} \neq 0$. This is strict rime.

If both $\gamma_{12}=0$ and $\gamma_{21}=0$ then equation (A.5) implies $\left(\alpha_{j i}+\beta_{i j}-\alpha_{j}\right)$ $\gamma_{j i}^{\prime}=0$; equation (A.7) implies $\left(\alpha_{i}-\alpha_{j}+\alpha_{j i}\right) \gamma_{j i}^{\prime}=0$ and equation (A.8) implies $\beta_{i j} \gamma_{j i}^{\prime}=0$. Combining these, we find $\gamma_{i j}^{\prime}=0$, this is ice.

It is left to analyze the situation when only one of $\gamma$ 's is different from zero, say $\gamma_{12} \neq 0$ and $\gamma_{21}=0$. We have the following chain of implications:

$$
\begin{gathered}
(\mathrm{A} .3) \Rightarrow \gamma_{12}^{\prime}=0, \\
(\mathrm{~A} .5) \Rightarrow\left\{\begin{array}{l}
\beta_{12}=\alpha_{2}-\alpha_{21}, \\
\beta_{21}=\alpha_{2}-\alpha_{12},
\end{array}\right. \\
\left(\text { A.4 } \Rightarrow\left(\alpha_{2}-\alpha_{12}\right)\left(\alpha_{2}-\alpha_{21}\right)=0,\right. \\
(\text { A. } 6) \Rightarrow\left\{\begin{array}{l}
\left(\alpha_{1}-\alpha_{2}\right)\left(\alpha_{2}-\alpha_{21}\right)\left(\alpha_{1}+\alpha_{21}\right)=0, \\
\left(\alpha_{1}-\alpha_{2}\right)\left(\alpha_{2}-\alpha_{12}\right)\left(\alpha_{1}+\alpha_{12}\right)=0,
\end{array}\right. \\
\qquad\left\{\begin{array}{l}
\left(\alpha_{1}-\alpha_{2}+\alpha_{12}\right) \gamma_{12}+\alpha_{12} \gamma_{21}^{\prime}=0, \\
\left(\alpha_{1}-\alpha_{2}+\alpha_{21}\right) \gamma_{21}^{\prime}+\alpha_{21} \gamma_{12}=0 .
\end{array}\right.
\end{gathered}
$$

Equations (A.8), (A.9) and (A.10) are satisfied. By the second line in (B.8), $\gamma_{21}^{\prime} \neq 0$.

Now the system of inequalities and equations is invariant under $\hat{R} \leftrightarrow \hat{R}_{21}$, so up to this transformation we can solve eq. (B.6) by setting $\alpha_{21}=\alpha_{2}$. Then, by (B.8), $\gamma_{21}^{\prime}=-\gamma_{12} \alpha_{2} / \alpha_{1}, \beta$ 's are expressed in terms of $\alpha$ 's by (B.5) and the remaining system for $\alpha$ 's reduces to a single equation $\left(\alpha_{1}-\alpha_{2}\right)\left(\alpha_{1}+\alpha_{12}\right)=0$. We obtain two solutions:

1a. $\alpha_{2}=\alpha_{1} ; \alpha_{1}, \alpha_{12}$ and $\gamma_{12}$ are arbitrary non-zero numbers; we rescale the $R$-matrix to set $\alpha_{1} \alpha_{12}=1$ and denote $q=\alpha_{1}, \gamma=\gamma_{12}$ :

$$
\hat{R}_{(q ; \gamma)}=\left(\begin{array}{cccc}
\alpha_{1} & 0 & 0 & 0 \\
\gamma_{12} & 0 & \alpha_{12} & 0 \\
-\gamma_{12} & \alpha_{1} & \alpha_{1}-\alpha_{12} & 0 \\
0 & 0 & 0 & \alpha_{1}
\end{array}\right)=\left(\begin{array}{cccc}
q & 0 & 0 & 0 \\
\gamma & 0 & q^{-1} & 0 \\
-\gamma & q & q-q^{-1} & 0 \\
0 & 0 & 0 & q
\end{array}\right)
$$

The $R$-matrix (B.9) is semi-simple if (and only if) $q+q^{-1} \neq 0$ and it is then an $R$-matrix of $\operatorname{GL}(2)$-type, $\operatorname{Spec}(\hat{R})=\left\{q, q, q,-q^{-1}\right\}$. This solution is a specialization of (1.15)-(1.16). 
1b. $\alpha_{12}=-\alpha_{1} ; \alpha_{1}, \alpha_{2}$ and $\gamma_{12}$ are arbitrary non-zero numbers; we rescale the $R$-matrix to set $\alpha_{1} \alpha_{2}=-1$ and denote $q=\alpha_{1}, \gamma=\gamma_{12} / q$ :

$$
\hat{R}_{(q ; \gamma)}=\left(\begin{array}{cccc}
\alpha_{1} & 0 & 0 & 0 \\
\gamma_{12} & 0 & -\alpha_{1} & 0 \\
-\gamma_{12} \alpha_{2} / \alpha_{1} & \alpha_{2} & \alpha_{1}+\alpha_{2} & 0 \\
0 & 0 & 0 & \alpha_{2}
\end{array}\right)=\left(\begin{array}{cccc}
q & 0 & 0 & 0 \\
q \gamma & 0 & -q & 0 \\
q^{-1} \gamma & -q^{-1} & q-q^{-1} & 0 \\
0 & 0 & 0 & -q^{-1}
\end{array}\right) .
$$

The $R$-matrix (B.10) is semi-simple if (and only if) $q+q^{-1} \neq 0$ and it is then an $R$-matrix of $\operatorname{GL}(1 \mid 1)$-type, $\operatorname{Spec}(\hat{R})=\left\{q, q,-q^{-1},-q^{-1}\right\}$.

2. Assume that $\alpha_{12}=0$.

By the invertibility, $\beta_{12} \beta_{21} \neq 0$; by the skew-invertibility, $\gamma_{12} \gamma_{12}^{\prime} \neq 0$; now equations (A.3) and (A.4) imply $\beta_{12} \beta_{21}=\gamma_{12} \gamma_{21}, \gamma_{12}^{\prime}=-\gamma_{21}$ and $\gamma_{21}^{\prime}=-\gamma_{12}$. Equation (A.5) implies $\alpha_{2}=\alpha_{1}, \beta_{12}=\alpha_{1}-\alpha_{21}$ and $\beta_{21}=\alpha_{1}$.

The rest is satisfied and we obtain a solution, in which $\alpha_{1}, \beta_{12}$ and $\gamma_{12}$ are arbitrary non-zero numbers; we rescale the $R$-matrix to set $\alpha_{1} \beta_{12}=-1$ and denote $q=\alpha_{1}, \gamma=\gamma_{12}$ :

$$
\hat{R}_{(q ; \gamma)}=\left(\begin{array}{cccc}
\alpha_{1} & 0 & 0 & 0 \\
\gamma_{12} & \beta_{12} & 0 & -\alpha_{1} \beta_{12} / \gamma_{12} \\
-\gamma_{12} & \alpha_{1}-\beta_{12} & \alpha_{1} & \alpha_{1} \beta_{12} / \gamma_{12} \\
0 & 0 & 0 & \alpha_{1}
\end{array}\right)=\left(\begin{array}{cccc}
q & 0 & 0 & 0 \\
\gamma & -q^{-1} & 0 & 1 / \gamma \\
-\gamma & q+q^{-1} & q & -1 / \gamma \\
0 & 0 & 0 & q
\end{array}\right) .
$$

The $R$-matrix (B.11) is semi-simple if (and only if) $q+q^{-1} \neq 0$ and it is then an $R$-matrix of $\operatorname{GL}(2)$-type, $\operatorname{Spec}(\hat{R})=\left\{q, q, q,-q^{-1}\right\}$. This solution is a specialization of (1.15)-(1.16).

3. Finally, assume that $\alpha_{12}=\alpha_{21}=0$.

By the invertibility, $\beta_{12} \beta_{21} \neq 0$; by the skew-invertibility, $\gamma_{12} \gamma_{12}^{\prime} \gamma_{21} \gamma_{21}^{\prime} \neq 0$; now equation (A.7) implies $\alpha_{2}=\alpha_{1}$, equation (A.9) implies $\beta_{21}=\beta_{12}$; equation (A.8) implies $\gamma_{12} \gamma_{12}^{\prime}=\gamma_{21} \gamma_{21}^{\prime}=-\alpha_{1} \beta_{12}$; equation (A.11) implies that $\gamma_{12} \gamma_{21}$ can take three values: $\alpha_{1}^{2}, \beta_{12}^{2}$ or $\left(-\alpha_{1} \beta_{12}\right)$.

The rest is satisfied and we obtain a solution, in which $\alpha_{1}, \beta_{12}$ and $\gamma_{12}$ are arbitrary non-zero numbers; we rescale the $R$-matrix to set $\alpha_{1} \beta_{12}=-1$ 
and denote $q=\alpha_{1}, \gamma=\gamma_{12}$ :

$$
\begin{aligned}
\hat{R}_{(q, \omega ; \gamma)} & =\left(\begin{array}{cccc}
\alpha_{1} & 0 & 0 & 0 \\
\gamma_{12} & \beta_{12} & 0 & -\alpha_{1} \beta_{12} / \gamma_{12} \\
-\alpha_{1} \beta_{12} \gamma_{12} / \omega & 0 & \beta_{12} & \omega / \gamma_{12} \\
0 & 0 & 0 & \alpha_{1}
\end{array}\right) \\
& =\left(\begin{array}{cccc}
q & 0 & 0 & 0 \\
\gamma & -q^{-1} & 0 & 1 / \gamma \\
\gamma / \omega & 0 & -q^{-1} & \omega / \gamma \\
0 & 0 & 0 & q
\end{array}\right)
\end{aligned}
$$

where $\omega=q^{2}, 1, q^{-2}$. The $R$-matrix (B.12) is semi-simple if (and only if) $q+q^{-1} \neq 0$ and it is then an $R$-matrix of $\operatorname{GL}(1 \mid 1)$-type, $\operatorname{Spec}(\hat{R})=\{q, q$, $\left.-q^{-1},-q^{-1}\right\}$.

It follows from the analysis above that if $\gamma_{i j} \neq 0$ in an invertible and skew-invertible rime $R$-matrix then $\gamma_{j i}^{\prime} \neq 0$.

In each of the cases (B.9)-(B.12), the parameter $\gamma \neq 0$ can be set to an arbitrary (non-zero) value by a diagonal change of basis. The $R$-matrices (B.9)-(B.12) are skew-invertible.

\section{B.2 GL(2) and GL(1|1) $R$-matrices}

1. In dimension 2, except the standard $R$-matrices of GL-type,

$$
\hat{R}_{(q, p)}^{\mathrm{GL}(2)}=\left(\begin{array}{cccc}
q & 0 & 0 & 0 \\
0 & 0 & p & 0 \\
0 & p^{-1} & q-q^{-1} & 0 \\
0 & 0 & 0 & q
\end{array}\right), \quad \hat{R}_{(q, p)}^{\mathrm{GL}(1 \mid 1)}=\left(\begin{array}{cccc}
q & 0 & 0 & 0 \\
0 & 0 & p & 0 \\
0 & p^{-1} & q-q^{-1} & 0 \\
0 & 0 & 0 & -q^{-1}
\end{array}\right)
$$

there are two non-standard one-parametric families of non-unitary $R$-matrices of the type GL(1|1): the eight-vertex one,

$$
\hat{R}_{(q)}^{I}=\frac{1}{2}\left(\begin{array}{cccc}
q-q^{-1}+2 & 0 & 0 & q-q^{-1} \\
0 & q-q^{-1} & q+q^{-1} & 0 \\
0 & q+q^{-1} & q-q^{-1} & 0 \\
q-q^{-1} & 0 & 0 & q-q^{-1}-2
\end{array}\right)
$$


and the matrix $\hat{R}^{(I I)}$ for which the matrix $R=P \hat{R}$ can be given an uppertriangular form,

$$
\hat{R}_{(q, \varepsilon)}^{I I}=\left(\begin{array}{cccc}
q & 0 & 0 & q+q^{-1} \\
0 & 0 & \varepsilon q^{-1} & 0 \\
0 & \varepsilon q & q-q^{-1} & 0 \\
0 & 0 & 0 & -q^{-1}
\end{array}\right)
$$

where $\varepsilon= \pm 1$.

The $R$-matrices (B.13), (B.14) and (B.15) are semi-simple if (and only if) $q+q^{-1} \neq 0$.

Up to the transformations $\hat{R} \leftrightarrow \hat{R}_{21}$ and $\hat{R} \leftrightarrow \hat{R}^{t}$ (the transposition), basis changes and rescalings $\hat{R} \mapsto c \hat{R}$ (where $c$ is a constant), the complete list of semi-simple invertible and skew-invertible $R$-matrices includes (see [16] for a description of all solutions of the Yang-Baxter equation in two dimensions and [11] for the classification of GL(2)-type $R$-matrices), in addition to (B.13)-(B.15), the one-parametric family of Jordanian solutions $\hat{R}_{\left(h_{1}: h_{2}\right)}^{(J)}$,

$$
\hat{R}_{\left(h_{1}: h_{2}\right)}^{(J)}=\left(\begin{array}{cccc}
1 & h_{1} & -h_{1} & h_{1} h_{2} \\
0 & 0 & 1 & -h_{2} \\
0 & 1 & 0 & h_{2} \\
0 & 0 & 0 & 1
\end{array}\right)
$$

(the Jordanian $R$-matrix is of GL(2)-type; it is unitary; the essential parameter is the projective vector $\left.\left(h_{1}: h_{2}\right)\right)$, as well as the permutation-like solution $\hat{R}_{(a, b, c)}^{(P)}$ and one more solution $\hat{R}_{(a)}^{\left({ }^{\prime}\right)}$,

$$
\hat{R}_{(a, b, c)}^{(P)}=\left(\begin{array}{cccc}
1 & 0 & 0 & 0 \\
0 & 0 & a & 0 \\
0 & b & 0 & 0 \\
0 & 0 & 0 & c
\end{array}\right), \quad \hat{R}_{(a)}^{\left({ }^{\prime}\right)}=\left(\begin{array}{cccc}
0 & 0 & 0 & a \\
0 & 1 & 0 & 0 \\
0 & 0 & 1 & 0 \\
a & 0 & 0 & 0
\end{array}\right) .
$$

The $R$-matrix $\hat{R}_{(a, b, c)}^{(P)}$ is Hecke when $a b=1$ and $c= \pm 1$ and it is then standard (and unitary). The $R$-matrix $\hat{R}_{(a)}^{\left({ }^{\prime}\right)}$ is Hecke when $a^{2}=1$; it is then unitary and related to the standard $R$-matrix by a change of basis with the matrix $\left(\begin{array}{cc}1 & 1 \\ -1 & 1\end{array}\right)$. 
Without the demand of semi-simplicity, the full list of invertible and skewinvertible $R$-matrices contains two more solutions,

$$
\left.\hat{R}_{\left(h_{1}: h_{2}: \sqrt{h_{3}}\right)}^{(\prime \prime)}=\left(\begin{array}{cccc}
1 & h_{1} & h_{2} & h_{3} \\
0 & 0 & 1 & h_{1} \\
0 & 1 & 0 & h_{2} \\
0 & 0 & 0 & 1
\end{array}\right), \quad \hat{R}^{(\prime \prime \prime}\right)=\left(\begin{array}{cccc}
1 & 0 & 0 & 1 \\
0 & 0 & -1 & 0 \\
0 & -1 & 0 & 0 \\
0 & 0 & 0 & 1
\end{array}\right) .
$$

The essential parameter for the $R$-matrix $\hat{R}_{\left(h_{1}: h_{2}: \sqrt{h_{3}}\right)}^{(\prime \prime}$ is the projective vector $\left(h_{1}: h_{2}: \sqrt{h_{3}}\right)$. The $R$-matrix $\hat{R}_{\left(h_{1}: h_{2}: \sqrt{h_{3}}\right)}^{(\prime \prime)}$ is semi-simple if and only if $h_{2}=-h_{1}$ and $h_{3}=-h_{1}^{2}$; it then belongs to the family (B.16) of Jordanian $R$-matrices.

2. For the $R$-matrices from the list above, the transformations $\hat{R} \leftrightarrow \hat{R}_{21}$, $\hat{R} \leftrightarrow \hat{R}^{t}$ and $\hat{R} \leftrightarrow \hat{R}^{-1}$ partly overlap or reduce to parameter or basis changes. We shall write formulas for the Hecke $R$-matrices only.

For the standard $R$-matrix $\hat{R}_{(q, p)}:=\hat{R}_{(q, p)}^{\mathrm{GL}(2)}$,

$$
\begin{aligned}
& \hat{R}_{(q, p)}^{t}=\hat{R}_{\left(q, p^{-1}\right)}, \quad\left(\hat{R}_{(q, p)}\right)_{21}=(\pi \otimes \pi) \hat{R}_{(q, p)}\left(\pi^{-1} \otimes \pi^{-1}\right) \\
& \hat{R}_{(q, p)}^{-1}=\left(\hat{R}_{\left(q^{-1}, p^{-1}\right)}\right)_{21},
\end{aligned}
$$

where $\pi=\left(\begin{array}{ll}0 & 1 \\ 1 & 0\end{array}\right)$.

For the standard $R$-matrix $\hat{R}_{(q, p)}:=\hat{R}_{(q, p)}^{\mathrm{GL}(1 \mid 1)}$,

$$
\begin{aligned}
& \hat{R}_{(q, p)}^{t}=\hat{R}_{\left(q, p^{-1}\right)}, \quad\left(\hat{R}_{(q, p)}\right)_{21}=(\pi \otimes \pi) \hat{R}_{\left(-q^{-1}, p\right)}\left(\pi^{-1} \otimes \pi^{-1}\right) \\
& \hat{R}_{(q, p)}^{-1}=\left(\hat{R}_{\left(q^{-1}, p^{-1}\right)}\right)_{21} .
\end{aligned}
$$

For the non-standard GL(1|1) $R$-matrix $\hat{R}_{(q)}:=\hat{R}_{(q)}^{I}$,

$$
\hat{R}_{(q)}^{t}=\hat{R}_{(q)}, \quad\left(\hat{R}_{(q)}\right)_{21}=\hat{R}_{(q)}, \quad \hat{R}_{(q)}^{-1}=(D \otimes D) \hat{R}_{\left(q^{-1}\right)}(D \otimes D)^{-1}
$$

where $D=\left(\begin{array}{cc}1 & 0 \\ 0 & \sqrt{-1}\end{array}\right)$. 
For the non-standard GL(1|1) $R$-matrix $\hat{R}_{(q, \varepsilon)}:=\hat{R}_{(q, \varepsilon)}^{I I}$,

$$
\hat{R}_{(q, \varepsilon)}^{t}=(\tilde{\pi} \otimes \tilde{\pi})\left(\hat{R}_{\left(-q^{-1},-\varepsilon\right)}\right)_{21}\left(\tilde{\pi}^{-1} \otimes \tilde{\pi}^{-1}\right), \quad \hat{R}_{(q, \varepsilon)}^{-1}=\left(\hat{R}_{\left(q^{-1}, \varepsilon\right)}\right)_{21},
$$

where $\tilde{\pi}=\left(\begin{array}{rr}0 & 1 \\ \sqrt{-1} & 0\end{array}\right)$.

For the Jordanian $R$-matrix $\hat{R}_{\left(h_{1}: h_{2}\right)}:=\hat{R}_{\left(h_{1}: h_{2}\right)}^{(J)}$,

$$
\begin{aligned}
\hat{R}_{\left(h_{1}: h_{2}\right)}^{t} & =(\pi \otimes \pi) \hat{R}_{\left(h_{2}: h_{1}\right)}\left(\pi^{-1} \otimes \pi^{-1}\right), \\
\left(\hat{R}_{\left(h_{1}: h_{2}\right)}\right)_{21} & =\hat{R}_{\left(-h_{1}:-h_{2}\right)}, \\
\hat{R}_{\left(h_{1}: h_{2}\right)}^{-1} & =\hat{R}_{\left(h_{1}: h_{2}\right)} .
\end{aligned}
$$

\section{B.3 Riming}

We shall now identify the rime $R$-matrices (B.9)-(B.12).

\section{GL(2)}

The $R$-matrices (B.9) and (B.11) are related by a change of basis (the number in brackets refers to the corresponding equation),

$$
\hat{R}_{(q ; \gamma)}^{(\mathrm{B} .9)} T \otimes T=T \otimes T \hat{R}_{(q ; \gamma)}^{(\mathrm{B} .11)}, \quad T=\left(\begin{array}{cc}
q & -1 / \gamma \\
\gamma & 0
\end{array}\right) .
$$

In turn, the $R$-matrix (B.9) is related to the standard $R$-matrix $\hat{R}_{\left(q, q^{-1}\right)}^{\mathrm{GL}(2)}$ by a change of basis,

$$
\hat{R}_{(q ; \gamma)}^{(\mathrm{B} .9)} T \otimes T=T \otimes T \hat{R}_{\left(q, q^{-1}\right)}^{\mathrm{GL}(2)}, \quad T=\left(\begin{array}{cc}
q-q^{-1} & 0 \\
\gamma & \gamma
\end{array}\right) .
$$

In the unitary situation (that is, $q-q^{-1}=0$ ), the $R$-matrix $\hat{R}_{(q ; \gamma)}^{(\mathrm{B} .9)}$ belongs to the family of Jordanian $R$-matrices.

Note that for the $R$-matrices (B.9) and (B.11), the left even quantum spaces are classical. 
2. $\mathrm{GL}(1 \mid 1)$

The $R$-matrix (B.10) is related to the $R$-matrix (B.12) with the choice $\omega=$ $\beta_{12}^{2}$,

$$
\hat{R}_{(q ; \gamma)}^{(\mathrm{B} .10)} T \otimes T=T \otimes T \hat{R}_{\left(-q^{-1}, q^{2} ; 1\right)}^{(\mathrm{B} .12)}, \quad T=\left(\begin{array}{cc}
1 & q \\
0 & \gamma q
\end{array}\right) .
$$

We have

$$
\begin{gathered}
\hat{R}_{(q, 1 ; \gamma)}^{\mathrm{B} .12)} T \otimes T=T \otimes T \hat{R}_{(q)}^{I}, \quad T=\left(\begin{array}{cc}
1 & \tau \\
\gamma & -\gamma \tau
\end{array}\right), \quad \text { where } \tau^{2}=\frac{q-1}{q+1}, \\
\hat{R}_{\left(q, q^{2} ; \gamma\right)}^{(\mathrm{B} 12)} T \otimes T=T \otimes T \hat{R}_{(q, 1)}^{I I}, \quad T=\left(\begin{array}{cc}
1 & 1 \\
\gamma q^{-1} & -\gamma q^{-1}
\end{array}\right), \\
\hat{R}_{\left(q, q^{-2} ; \gamma\right)}^{(\mathrm{B} .12)} T \otimes T=T \otimes T\left(\hat{R}_{(q, 1)}^{I I}\right)_{21}, \quad T=\left(\begin{array}{cc}
1 & 1 \\
\gamma q & -\gamma q
\end{array}\right) .
\end{gathered}
$$

In the unitary situation (that is, for $q= \pm 1$ ) only equation (B.27) changes; but now different choices for $\omega$ coincide.

3. Since the standard $R$-matrices are rime as well, we conclude that in dimension 2, all non-unitary Hecke $R$-matrices fit into the rime Ansatz. When $h_{1}=0$, the Jordanian $R$-matrix $\hat{R}_{\left(0: h_{2}\right)}^{(J)}$ is rime as well. However, when $h_{1} \neq 0$, the Jordanian $R$-matrix $\hat{R}_{\left(h_{1}: h_{2}\right)}^{(J)}$ cannot be rimed. Indeed, assume that $h_{1} \neq 0$ and let $A=(T \otimes T) \hat{R}_{\left(h_{1}: h_{2}\right)}^{(J)}(T \otimes T)^{-1}$ with some invertible matrix $T$. Then

$$
\begin{aligned}
& (\operatorname{Det}(T))^{2} A_{12}^{11}=h_{1}\left(T_{1}^{1}\right)^{2}\left(\operatorname{Det}(T)-h_{2} T_{1}^{1} T_{1}^{2}\right), \\
& (\operatorname{Det}(T))^{2} A_{21}^{11}=-h_{1}\left(T_{1}^{1}\right)^{2}\left(\operatorname{Det}(T)+h_{2} T_{1}^{1} T_{1}^{2}\right), \\
& (\operatorname{Det}(T))^{2} A_{12}^{22}=h_{1}\left(T_{1}^{2}\right)^{2}\left(\operatorname{Det}(T)-h_{2} T_{1}^{1} T_{1}^{2}\right), \\
& (\operatorname{Det}(T))^{2} A_{21}^{11}=-h_{1}\left(T_{1}^{2}\right)^{2}\left(\operatorname{Det}(T)+h_{2} T_{1}^{1} T_{1}^{2}\right) .
\end{aligned}
$$

For an invertible $T$, the non-rime entries (B.30) of $A$ cannot vanish simultaneously.

4. We remark also that all non-standard $R$-matrices of GL(1|1)-type are uniformly described by the formula (B.12). The right quantum spaces for 
the $R$-matrix $\hat{R}_{(q, \omega ; \gamma)}^{(\mathrm{B} .12)}$, with $\gamma=1$, read

$$
\begin{aligned}
\left(\hat{R}-q \mathbb{1} \otimes \mathbb{1}_{k l}^{i j} x^{k} x^{l}=0:\left\{\begin{array}{l}
\left(q+q^{-1}\right) x y=x^{2}+y^{2}, \\
\left(q+q^{-1}\right) x y=\omega^{-1} x^{2}+\omega y^{2} ;
\end{array}\right.\right. \\
\left(\hat{R}+q^{-1} \mathbb{1} \otimes \mathbb{1}\right)_{k l}^{i j} x^{k} x^{l}=0:\left\{\begin{array}{l}
x^{2}=0, \\
y^{2}=0 .
\end{array}\right.
\end{aligned}
$$

Using the diamond lemma, it is straightforward to verify that the Poincaré series of the quantum space (B.31) is of GL(1|1)-type if and only if $\omega=q^{-2}$, 1 or $q^{2}$.

\section{Appendix C. Rimeless triple}

We sketch here a proof that the triple (4.18) cannot be rimed. Relations $x^{i} y^{j}=\hat{R}_{k l}^{i j} y^{k} x^{l}$, where $\hat{R}$ is the $R$-matrix for the triple (4.18) with arbitrary multiparameters, read

$$
x^{i} y^{i}=y^{i} x^{i}, \quad i=1,2,3,4
$$

and

$$
\begin{gathered}
x^{1} y^{2}=\frac{p}{q} y^{2} x^{1}, \quad x^{2} y^{1}=\frac{1}{p q} y^{1} x^{2}+\left(1-q^{-2}\right) y^{2} x^{1}, \\
x^{1} y^{3}=\frac{r}{q^{2}} y^{3} x^{1}, \quad x^{2} y^{3}=\frac{s}{p q} y^{3} x^{2}, \\
x^{1} y^{4}=\frac{p r}{q} y^{4} x^{1}-\frac{r s}{q} y^{3} x^{2}, \quad x^{2} y^{4}=\frac{s}{q^{2}} y^{4} x^{2}, \\
x^{3} y^{1}=\frac{1}{r} y^{1} x^{3}+\left(1-q^{-2}\right) y^{3} x^{1}, \quad x^{4} y^{1}=\frac{1}{p q r} y^{1} x^{4}+\left(1-q^{-2}\right) y^{4} x^{1}+\frac{1}{q} y^{2} x^{3}, \\
x^{3} y^{2}=\frac{p}{q s} y^{2} x^{3}+\left(1-q^{-2}\right) y^{3} x^{2}, \quad x^{4} y^{2}=\frac{1}{s} y^{2} x^{4}+\left(1-q^{-2}\right) y^{4} x^{2}, \\
x^{3} y^{4}=\frac{p r}{q s} y^{4} x^{3}, \quad x^{4} y^{3}=\frac{s}{p q r} y^{3} x^{4}+\left(1-q^{-2}\right) y^{4} x^{3} .
\end{gathered}
$$


The parameter $q$ enters the characteristic equation for $\hat{R}, \hat{R}^{2}=\left(1-q^{-2}\right) \hat{R}+$ $q^{-2} \mathbb{1} \otimes \mathbb{1} ; p, r$ and $s$ are the multiparameters. The only needed restriction is $q^{2} \neq 1$.

Denote by $\left\langle l^{(1)}, l^{(2)}\right\rangle$ a two-dimensional plane spanned by $l^{(1)}$ and $l^{(2)}$. We say that two linear forms $l^{(1)}$ and $l^{(2)}$ (in four variables) form a rime pair if, for the ordering relations (C.1) and (C.2)-(C.3), each product $l^{(\alpha)}(x) l^{(\beta)}(y)$, $\alpha=1,2, \beta=1,2$, is a linear combination of $l^{(1)}(y) l^{(1)}(x), l^{(1)}(y) l^{(2)}(x)$, $l^{(2)}(y) l^{(1)}(x)$ and $l^{(2)}(y) l^{(2)}(x)$. If, in addition, $l^{(\alpha)}(x) l^{(\alpha)}(y)$ is proportional to $l^{(\alpha)}(y) l^{(\alpha)}(x)$ for $\alpha=1$ and 2, we say that $l^{(1)}$ and $l^{(2)}$ form a rime basis in the plane $\left\langle l^{(1)}, l^{(2)}\right\rangle$. We call a plane rime if it admits a rime basis.

Fork Lemma. Assume that $l^{(1)}(x)=x^{1}+a_{2} x^{2}+a_{3} x^{3}$ and $l^{(4)}(x)=b_{2} x^{2}+$ $b_{3} x^{3}+x^{4}$ form a rime pair for some $a_{2}, a_{3}, b_{2}$ and $b_{3}$. Then either $a_{3} b_{2} \neq 0$ and $a_{2}=b_{3}=0$ or $a_{2} b_{3} \neq 0$ and $a_{3}=b_{2}=0$.

If $a_{3} b_{2} \neq 0$ then

$r=s=1, \quad l^{(1)}(x)=x^{1}+w x^{3}$ and $l^{(4)}(x)=x^{4}+\frac{1}{q-q^{-1}} \frac{1}{w} x^{2}$, $w \neq 0$ is arbitrary.

If $a_{2} b_{3} \neq 0$ then

$p=\frac{1}{q}, \quad r=s, \quad l^{(1)}(x)=x^{1}+w x^{2}$ and $l^{(4)}(x)=x^{4}+\frac{s}{q-q^{-1}} \frac{1}{w} x^{3}$, $w \neq 0$ is arbitrary.

Moreover, if $r=s=1$ and $p \neq q^{-1}$ then the rime plane $\left\langle l^{(1)}, l^{(4)}\right\rangle$ admits a unique, up to rescalings, rime basis $\left\{l^{(1)}, l^{(4)}\right\}$; if $p=q^{-1}$ and $r=s \neq 1$ then the rime plane $\left\langle l^{(1)}, l^{(4)}\right\rangle$ admits a unique, up to rescalings, rime basis $\left\{l^{(1)}, l^{(4)}\right\}$; if $p=q^{-1}$ and $r=s=1$ then any two independent linear combinations of $l^{(1)}$ and $l^{(4)}$ form a rime basis in the plane $\left\langle l^{(1)}, l^{(4)}\right\rangle$.

Proof. A straightforward calculation.

Assume that a rime basis $\left\{\tilde{x}^{i}\right\}$ for the triple (4.18) exists, $\tilde{x}^{i}=A_{j}^{i} x^{j}$, the matrix $A_{j}^{i}$ is invertible. Rename the rime variables $\tilde{x}^{i}$ in such a way that the minor $\left|\begin{array}{ll}A_{1}^{1} & A_{4}^{1} \\ A_{1}^{4} & A_{4}^{4}\end{array}\right|$ is non-zero and $A_{1}^{1} A_{4}^{4} \neq 0$; normalize the variables $\tilde{x}^{1}$ and 
$\tilde{x}^{4}$ to have $A_{1}^{1}=A_{4}^{4}=1$. The plane $\left\langle\tilde{x}^{1}, \tilde{x}^{4}\right\rangle$ is, by definition, rime, with a rime basis $\left\{\tilde{x}^{1}, \tilde{x}^{4}\right\}$.

Suppose that $r=s=1$ and $p \neq q^{-1}$ or $p=q^{-1}$ and $r=s \neq 1$. Then, by Fork Lemma, the rime basis in the plane $\left\langle\tilde{x}^{1}, \tilde{x}^{4}\right\rangle$ is, up to proportionality, unique, so we know the variables $\tilde{x}^{1}$ and $\tilde{x}^{4}$. The variables $\tilde{x}^{1}$ and $\tilde{x}^{2}$ form a rime plane. Therefore, if the variable $\tilde{x}^{2}$ contains $x^{4}$ with a non-zero coefficient then, by Fork Lemma, $\tilde{x}^{2}$ must be proportional to $\tilde{x}^{4}$, contradicting to the linear independence of the variables $\tilde{x}^{2}$ and $\tilde{x}^{4}$. Similarly, the variable $\tilde{x}^{2}$ cannot contain $x^{1}$ with a non-zero coefficient (the plane $\left\langle\tilde{x}^{2}, \tilde{x}^{4}\right\rangle$ is rime). Thus, $\tilde{x}^{2}$ is a linear combination of $x^{2}$ and $x^{3}$. Same for $\tilde{x}^{3}$ : it is a linear combination of $x^{2}$ and $x^{3}$. One of the variables, $\tilde{x}^{2}$ or $\tilde{x}^{3}$, say, $\tilde{x}^{2}$, contains $x^{2}$ with a non-zero coefficient. Writing rime equations for the plane $\left\langle\tilde{x}^{1}, \tilde{x}^{2}\right\rangle$ in the case $r=s=1$ and $p \neq q^{-1}$ (for the plane $\left\langle\tilde{x}^{2}, \tilde{x}^{4}\right\rangle$ in the case $p=q^{-1}$ and $r=s \neq 1$ ) quickly leads to a contradiction.

Therefore, if the relations (C.1) and (C.2)-(C.3) can be rimed then $p=q^{-1}$ and $r=s=1$. It follows from Fork Lemma that

$$
\tilde{x}^{4}=\left(q-q^{-1}\right) c_{2} c_{3} x^{1}+c_{2} x^{2}+c_{3} x^{3}+x^{4}
$$

for some $c_{2}$ and $c_{3}$. The planes $\left\langle\tilde{x}^{a}, \tilde{x}^{4}\right\rangle, a=1,2,3$, are rime. Subtracting from the variables $\tilde{x}^{a}$ the variable $\tilde{x}^{4}$ with appropriate coefficients, we find three linearly independent combinations

$$
l(x)=d_{1} x^{1}+d_{2} x^{2}+d_{3} x^{3}
$$

each forming a rime pair with $\tilde{x}^{4}$. We must have: $l(x) l(y)$ is a linear combination of $l(y) l(x), l(y) \tilde{x}^{4}, \tilde{y}^{4} l(x)$ and $\tilde{y}^{4} \tilde{x}^{4}$. It follows, after a straightforward calculation, that $d_{2} d_{3}=0$. Moreover, $d_{2}=d_{3}=0$ is excluded by Fork Lemma. In the case $d_{2} \neq 0$ and $d_{3}=0$ (respectively, $d_{3} \neq 0$ and $d_{2}=$ 0 ), the rime condition implies that $d_{1}=\left(q-q^{-1}\right) c_{2} d_{3}$ (respectively, $d_{1}=$ $\left.\left(q-q^{-1}\right) c_{3} d_{2}\right)$. Thus, only two linearly independent combinations (C.6) can form a rime pair with $\tilde{x}^{4}$, the final contradiction.

\section{References}

[1] M. Aguiar, Infinitesimal Hopf algebras, Contemp. Math. 267 (2000), $1-30$.

[2] G. E. Arutyunov and S. A. Frolov, Quantum dynamical R-matrices and quantum Frobenius group, Comm. Math. Phys. 191 (1998), 15-29, ArXiv:q-alg/9610009. 
[3] A. A. Belavin and V. G. Drinfeld, Triangle equations and simple Lie algebras, Sov. Sci. Rev. C4 (1984), 93-166.

[4] A. A. Belavin and V. G. Drinfeld, Solutions of the classical Yang-Baxter equation for simple Lie algebras, Funct. Anal. Appl. 16 (1982), 159-180.

[5] E. Bézout, Recherches sur le degré des équations résultantes de l'évanouissement des inconnnues, et sur les moyens qu'il convient d'employer pour trouver ces équations, Histoire de l'Académie Royale des Sciences, Année MDCCLXIV, Avec les Mémoires de Mathématique \& de Physique, pour la même Année, Tirés des Registres de cette Académie, Paris, 1767, 288-338.

[6] E. Cremmer and J.-L. Gervais, The quantum group structure associated with non-linearly extended Virasoro algebras, Comm. Math. Phys. 134 (1990), 619-632.

[7] M. Demazure, Une nouvelle formule des caractères, Bull. Sci. Math. (2) 98(3) (1974), 163-172.

[8] R. Endelman and T. Hodges, Generalized Jordanian R-matrix of Cremmer-Gervais type, Lett. Math. Phys. 52 (2000), 225-237, ArXiv: math.QA/0003066.

[9] P. Etingof, T. Schedler and O. Schiffmann, Explicit quantization of dynamical r-matrices for finite dimensional semisimple Lie algebras, J. Amer. Math. Soc. 13(3) (2000), 595-609, ArXiv: math.QA/9912009.

[10] H. Ewen and O. Ogievetsky, Classification of the GL(3) quantum matrix groups, ArXiv:q-alg/9412009.

[11] H. Ewen, O. Ogievetsky and J. Wess, Quantum matrices in two dimensions, Lett. Math. Phys. 22 (1991), 297-305.

[12] L. Fehér and B. G. Pusztai, On the classical R-matrix of the degenerate Calogero-Moser models, Czech. J. Phys. 50 (2000), 59-64, ArXiv: math-ph/9912021.

[13] L. Fehér and B. G. Pusztai, The non-dynamical r-matrices of the degenerate Calogero-Moser models, J. Phys. A33 (2000), 7739-7759, ArXiv: math-ph/0005021.

[14] M. Gerstenhaber and A. Giaquinto, Boundary solutions of the classical Yang-Baxter equation, Lett. Math. Phys. 40 (1997), 337-353, ArXiv: q-alg/9609014.

[15] V. Gorbounov, A. Isaev and O. Ogievetsky, BRST Operator for quantum Lie algebras: relation to bar complex, Teoret. Mat. Fiz., 139(1) (2004), 29-44, translation in: Theoret. Math. Phys. 139(1) (2004), $473-485$. 
[16] J. Hietarinta, Solving the two-dimensional constant quantum Yang-Baxter equation, J. Math. Phys. 34(5) (1993), 1725-1756.

[17] T. Hodges, The Cremmer-Gervais solution of the Yang-Baxter equation, Proc. Amer. Math. Soc. 127(6) (1999), 1819-1826, ArXiv: q-alg/9712036.

[18] A. Isaev and O. Ogievetsky, On quantization of r-matrices for BelavinDrinfeld triples, Phys. Atomic Nuclei 64(12) (2001), 2126-2130, ArXiv:math.QA/0010190.

[19] G. Lusztig, Equivariant K-theory and representations of Hecke algebras, Proc. Amer. Math. Soc. 94(2) (1985), 337-342.

[20] A. Mudrov, Associative triples and Yang-Baxter equation, Israel J. Math. 139 (2004), 11-28, ArXiv:math.QA/0003050.

[21] O. Ogievetsky, Uses of quantum spaces, in "Quantum Symmetries in Theoretical Physics and Mathematics", edited by R. Coquereaux, A. Garcia and R. Trinchero, Contemp. Math. 294, Amer. Math. Soc., Providence, RI 2002, 161-232.

[22] G. C. Rota, Baxter operators, an introduction, in Gian-Carlo rota on combinatorics, Contemp. Math, Birkhäuser Boston, Boston, MA 1995, 504-512.

[23] M. A. Semenov-Tyan-Shanskii, What is a classical r-matrix, Funktsional. Analiz i Prilozhen. 17(4) (1983), 17-33.

[24] A. Stolin, On the rational solutions of the classical Yang-Baxter equation, Ph. D. Thesis, Stockholm, 1991. 
